\title{
From Cycle Rooted Spanning Forests to the Critical Ising Model: an Explicit Construction
}

\author{
Béatrice de Tilière *
}

\begin{abstract}
Fisher established an explicit correspondence between the 2-dimensional Ising model defined on a graph $G$ and the dimer model defined on a decorated version $\mathcal{G}$ of this graph [Fis66]. In this paper we explicitly relate the dimer model associated to the critical Ising model and critical cycle rooted spanning forests (CRSFs). This relation is established through characteristic polynomials, whose definition only depends on the respective fundamental domains, and which encode the combinatorics of the model. We first show a matrix-tree type theorem establishing that the dimer characteristic polynomial counts CRSFs of the decorated fundamental domain $\mathcal{G}_{1}$. Our main result consists in explicitly constructing CRSFs of $\mathcal{G}_{1}$ counted by the dimer characteristic polynomial, from CRSFs of $G_{1}$ where edges are assigned Kenyon's critical weight function [Ken02]; thus proving a relation on the level of configurations between two well known 2-dimensional critical models.
\end{abstract}

\section{Introduction}

In [Fis66], Fisher established an explicit correspondence between the 2-dimensional Ising model defined on a graph $G$ and the dimer model defined on a decorated version $\mathcal{G}$ of this graph, known as the Fisher graph of $G$. Since then, dimer techniques have been a powerful tool for tackling the Ising model, see for example the book of [MW73]. More recently, in [BdT10b, BdT10a], we prove fundamental results for the dimer model corresponding to a large class of critical Ising models, by proving explicit formulae for the free energy and for the Gibbs measure.

Critical Ising models we consider are defined on graphs satisfying a geometric property called isoradiality. When the underlying isoradial graph $G$ is infinite and $\mathbb{Z}^{2}$-periodic,

${ }^{*}$ Laboratoire de Probabilités et Modèles Aléatoires, Université Pierre et Marie Curie, 4 place Jussieu, F-75005 Paris. beatrice.de_tiliere@upmc.fr. Institut de Mathématiques, Université de Neuchâtel, Rue Emile-Argand 11, CH-2007 Neuchâtel. Supported in part by the Swiss National Foundations grant 200020-120218. 
then so is the Fisher graph $\mathcal{G}$, and we let $\mathcal{G}_{1}=\mathcal{G} / \mathbb{Z}^{2}$ be the fundamental domain. The key object involved in the explicit expressions of [BdT10b] for the free energy and the Gibbs measure is the critical dimer characteristic polynomial, whose definition only depends on the fundamental domain $\mathcal{G}_{1}$. This polynomial is a generating function for configurations related to super-imposed dimer configurations of $\mathcal{G}_{1}$, referred to as 'double-dimer' configurations. By Fisher's correspondence, this implies that the dimer characteristic polynomial is a generating function for 'double-Ising' configurations.

In [BdT10b], we prove that the dimer characteristic polynomial is equal, up to a constant, to the critical Laplacian characteristic polynomial of $G_{1}=G / \mathbb{Z}^{2}$, where edges of $G_{1}$ are assigned Kenyon's critical weight function [Ken02]. Using a generalization of Kirchhoff's matrix tree theorem due to Forman [For93], the latter is shown to be a generating function for cycle rooted spanning forests (CRSFs) of $G_{1}$. This suggests the existence of an explicit relation between 'double-Ising' configurations and CRSFs, which we were not able to find in [BdT10b]. The first result of this paper is a matrix-tree type theorem, proving that the critical dimer characteristic polynomial is a generating function for CRSFs of the Fisher graph $\mathcal{G}_{1}$, see Theorem 7 of Section 4.2. Then, the main result of this paper can loosely be stated as follows, refer to Theorem 17 of Section 4.4 and Theorem 29 of Section 6.1 for more precise statements.

Theorem 1. Consider a critical Ising model defined on an infinite, $\mathbb{Z}^{2}$-periodic isoradial graph $G$. Then, there exists an explicit way of constructing CRSFs of $\mathcal{G}_{1}$ counted by the critical dimer characteristic polynomial, from CRSFs of $G_{1}$ counted by the critical Laplacian characteristic polynomial.

This exhibits an explicit relation, on the level of configurations, between two well known models of statistical mechanics: the Ising model and CRSFs at criticality. Note that such a relation was already suspected by Messikh [Mes06]. Before giving an outline of the paper, let us make a few comments.

- The main contribution of this paper is the proof of Theorem 1, where we actually provide the explicit construction.

- The partition functions (weighted sum of configurations) of both models can be expressed from their respective characteristic polynomials, so that it is actually stronger to work with characteristic polynomials.

- Working with graphs embedded on the torus has the advantage of avoiding boundary issues, but has the additional difficulty of involving the geometry of the torus, with non-trivial cycles occurring in configurations. Working on finite pieces of infinite graphs, and precisely specifying boundary conditions, would certainly explicitly relate double-dimer configurations and spanning trees.

- Spanning trees are a well suited object for defining a height function and prove Gaussian fluctuations. Thus, it might be that Theorem 1 could be used to prove results which are numerically described in the paper [Wil11]. 


\section{Outline of the paper}

Section 2: Definition of the critical Ising model and of the dimer model. Description of Fisher's correspondence relating the two.

Section 3: Definition of the critical dimer and Laplacian characteristic polynomials. Relation between the Laplacian characteristic polynomial and CRSFs.

Section 4: Statement and proof of Theorem 7 establishing that the critical dimer characteristic polynomial is a generating function for CRSFs of the Fisher graph $\mathcal{G}_{1}$. Precise statement of Theorem 1.

Section 5: Definition and properties of licit primal/dual edge moves, which are one of the key ingredients of the correspondence.

Section 6: Explicit construction of CRSFs of $\mathcal{G}_{1}$ from CRSFs of $G_{1}$ and proof of Theorem 1.

\section{Critical Ising model and dimer model}

In this section, we define the 2-dimensional critical Ising model, the dimer model and describe Fisher's correspondence relating the two.

\section{$2.1 \quad$ Critical Ising model}

Let $G=(V(G), E(G))$ be a finite, planar, unoriented graph, together with a collection of positive real numbers $J=\left(J_{e}\right)_{e \in E(G)}$ indexed by the edges of $G$. The Ising model on $G$ with coupling constants $J$ is defined as follows. A spin configuration $\sigma$ of $G$ is a function of the vertices of $G$ with values in $\{-1,+1\}$. The probability of occurrence of a spin configuration $\sigma$ is given by the Ising Boltzmann measure, denoted $\mathbb{P}_{\text {Ising }}$ :

$$
\mathbb{P}_{\text {Ising }}(\sigma)=\frac{1}{Z_{\text {Ising }}} \exp \left(\sum_{e=u v \in E(G)} J_{e} \sigma_{u} \sigma_{v}\right),
$$

where $Z_{\text {Ising }}=\sum_{\sigma \in\{-1,1\}^{V(G)}} \exp \left(\sum_{e=u v \in E(G)} J_{e} \sigma_{u} \sigma_{v}\right)$, is the Ising partition function. We consider Ising models defined on a class of embedded graphs which have an additional property called isoradiality. A graph $G$ is said to be isoradial [Ken02], if it has an embedding in the plane such that every face is inscribed in a circle of radius 1 . We ask moreover that all circumcenters of the faces are in the closure of the faces. From now on, when we speak of the graph $G$, we mean the graph together with a particular isoradial embedding in the plane. Examples of isoradial graphs are the square and the honeycomb lattice. Refer to Figure 1 (left) for a more general example of isoradial graph. 
To such a graph is naturally associated the diamond graph, denoted by $G^{\diamond}$ : vertices of $G^{\diamond}$ consist in the vertices of $G$ and the circumcenters of the faces of $G$ (which are also the dual vertices of $G^{*}$ ); the circumcenter of each face is then joined to all vertices which are on the boundary of this face, see Figure 1 (center). Since $G$ is isoradial, all faces of $G^{\diamond}$ are side-length-1 rhombi. Moreover, each edge $e$ of $G$ is the diagonal of exactly one rhombus of $G^{\diamond}$; we let $\theta_{e}$ be the half-angle of the rhombus at the vertex it has in common with $e$, see Figure 1 (right).
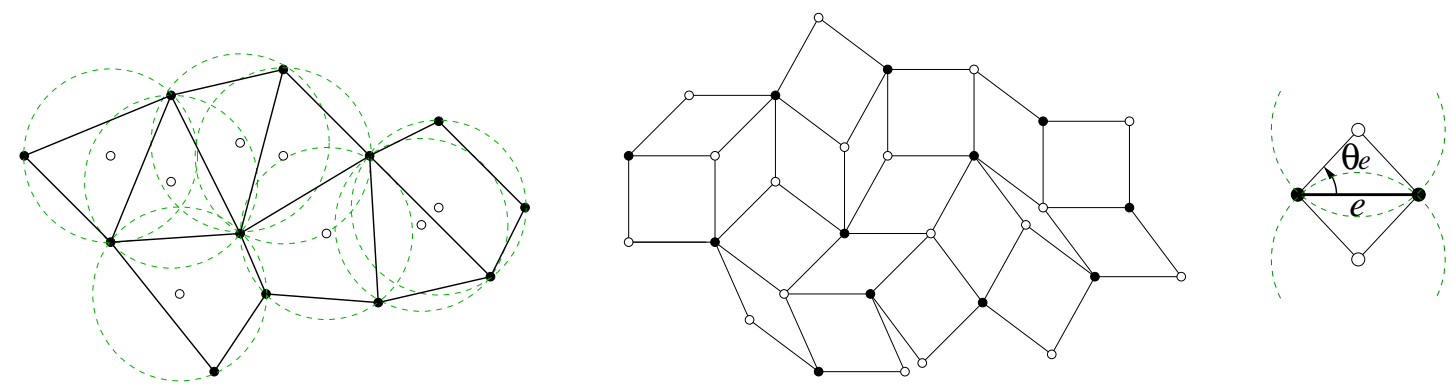

Figure 1: Left: example of isoradial graph. Center: corresponding diamond graph. Right: rhombus half-angle associated to an edge $e$ of the graph.

The same construction can be done for infinite and toroidal isoradial graphs, in which case the embedding is in the plane or in the torus.

It is then natural to choose the coupling constants $J$ of the Ising model to depend on the geometry of the embedded graph: let us assume that $J_{e}$ is a function of $\theta_{e}$, the rhombus half-angle assigned to the edge $e$.

We impose two more conditions on the coupling constants. First, we ask that the Ising model on $G$ with coupling constants $J$ as above is $Z$-invariant, that is, invariant under star-triangle transformations of the underlying graph. Next, we impose that the Ising model satisfies a generalized form of self-duality. These conditions completely determine the coupling constants $J$, known as critical coupling constants: for every edge $e$ of $G$,

$$
J\left(\theta_{e}\right)=\frac{1}{2} \log \left(\frac{1+\sin \theta_{e}}{\cos \theta_{e}}\right) .
$$

The $Z$-invariant Ising model on an isoradial graph with this particular choice of coupling constants is referred to as critical Ising model. This model was introduced by Baxter in [Bax86]. A more detailed definition is given in [BdT10b].

\subsection{Dimer model}

Let $\mathcal{G}=(V(\mathcal{G}), E(\mathcal{G}))$ be a finite, planar, unoriented graph, and suppose that edges of $\mathcal{G}$ are assigned a positive weight function $\nu=\left(\nu_{e}\right)_{e \in E(\mathcal{G})}$. The dimer model on $\mathcal{G}$ with weight function $\nu$ is defined as follows. 
A dimer configuration $M$ of $\mathcal{G}$, also called perfect matching, is a subset of edges of $\mathcal{G}$ such that every vertex is incident to exactly one edge of $M$. Let $\mathcal{M}(\mathcal{G})$ be the set of dimer configurations of the graph $\mathcal{G}$. The probability of occurrence of a dimer configuration $M$ is given by the dimer Boltzmann measure, denoted $\mathbb{P}_{\text {dimer }}$ :

$$
\mathbb{P}_{\text {dimer }}(M)=\frac{\prod_{e \in M} \nu_{e}}{Z_{\text {dimer }}}
$$

where $Z_{\text {dimer }}=\sum_{M \in \mathcal{M}(\mathcal{G})} \prod_{e \in M} \nu_{e}$ is the dimer partition function.

\subsection{Fisher's correspondence}

Fisher's correspondence [Fis66] holds for a general Ising model defined on a finite graph $G$ embedded on a surface without boundary, with coupling constants $J$. We use the following slight variation of the correspondence.

The decorated graph, on which the dimer configurations live, is constructed from $G$ as follows. Every vertex of degree $k$ of $G$ is replaced by a decoration consisting of $3 k$ vertices: a triangle is attached to every edge incident to this vertex, and these triangles are joined by edges in a circular way, see Figure 2 below. This new graph, denoted by $\mathcal{G}$, is also embedded on the surface without boundary and has vertices of degree 3 . It is referred to as the Fisher graph of $G$.
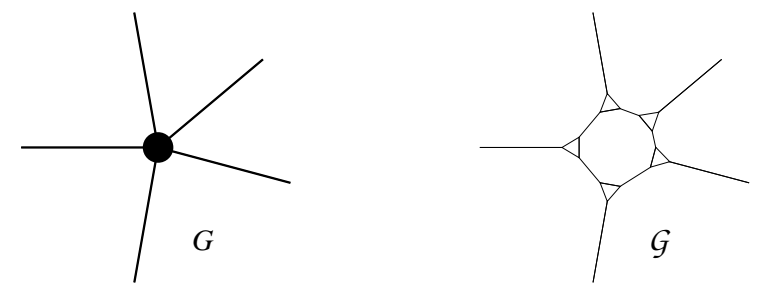

Figure 2: Left: a vertex of $G$ with its incoming edges. Right: corresponding decoration of $\mathcal{G}$.

Fisher's correspondence uses the high temperature expansion of the Ising partition function, see for example [Bax89]:

$$
Z_{\text {Ising }}=\left(\prod_{e \in E(G)} \cosh \left(J_{e}\right)\right) 2^{|V(G)|} \sum_{\mathcal{C} \in \mathcal{P}} \prod_{e \in \mathcal{C}} \tanh \left(J_{e}\right),
$$

where $\mathcal{P}$ is the family of all polygonal contours drawn on $G$, for which every edge of $G$ is used at most once. This expansion defines a measure on the set of polygonal contours $\mathcal{P}$ of $G$ : the probability of occurrence of a polygonal contour $\mathcal{C}$ is proportional to the product of the weights of the edges it contains, where the weight of an edge $e$ is $\tanh \left(J_{e}\right)$. 
Here comes the correspondence: to any contour configuration $\mathcal{C}$ coming from the hightemperature expansion of the Ising model on $G$, we associate $2^{|V(G)|}$ dimer configurations on $\mathcal{G}$ : edges present (resp. absent) in $\mathcal{C}$ are absent (resp. present) in the corresponding dimer configuration of $\mathcal{G}$. Once the state of these edges is fixed, there is, for every decorated vertex, exactly two ways to complete the configuration into a dimer configuration. Figure 3 below gives an example in the case where $G$ is the square lattice $\mathbb{Z}^{2}$.
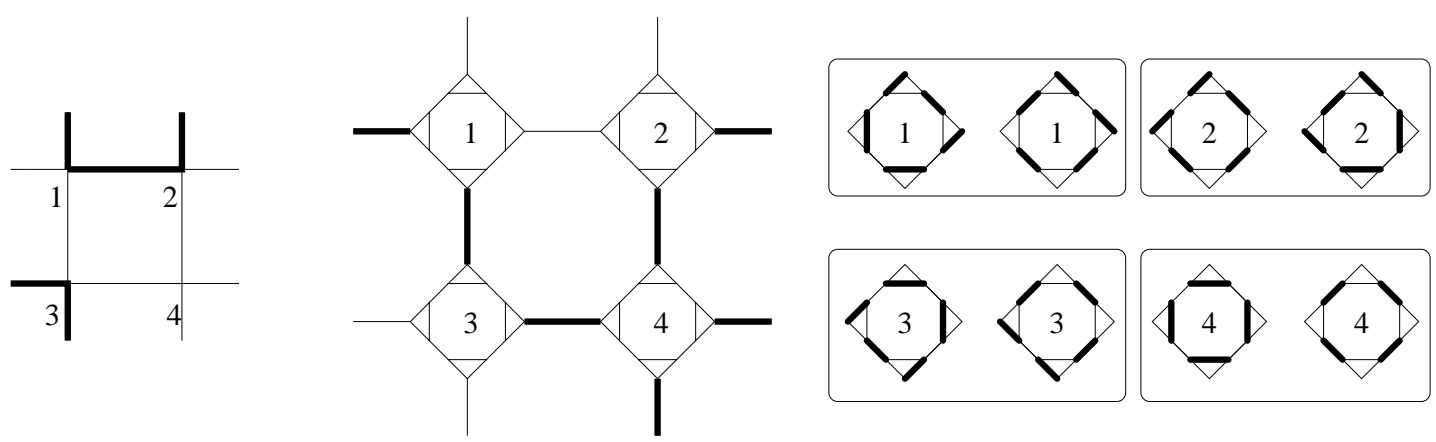

Figure 3: Polygonal contour of $\mathbb{Z}^{2}$, and corresponding dimer configurations of the associated Fisher graph.

Let us assign, to an edge $e$ of $\mathcal{G}$, weight $\nu_{e}=1$, if it belongs to a decoration; and weight $\nu_{e}=\operatorname{coth} J_{e}$, if it corresponds to an edge of $G$. Then the correspondence is measurepreserving: every contour configuration $\mathcal{C}$ has the same number $\left(2^{|V(G)|}\right)$ of images by this correspondence, and the product of the weights of the edges in $\mathcal{C}, \prod_{e \in \mathcal{C}} \tanh \left(J_{e}\right)$ is proportional to the weight $\prod_{e \notin \mathcal{C}} \operatorname{coth}\left(J_{e}\right)$ of any of its corresponding dimer configurations for a proportionality factor, $\prod_{e \in E(G)} \tanh \left(J_{e}\right)$, which is independent of $\mathcal{C}$.

As a consequence of Fisher's correspondence, we have the following relation between the Ising and dimer partition functions:

$$
Z_{\text {Ising }}=\left(\prod_{e \in E(G)} \sinh \left(J_{e}\right)\right) Z_{\text {dimer }} .
$$

Fisher's correspondence between Ising contour configurations and dimer configurations naturally extends to the case where $G$ is an infinite planar graph.

\subsection{Critical dimer model on Fisher graphs}

Consider a critical Ising model defined on an isoradial graph $G$ embedded in the torus, or in the plane. Then, the dimer weights of the corresponding dimer model on the 
Fisher graph $\mathcal{G}$ are:

$$
\nu_{e}= \begin{cases}1 & \text { if } e \text { belongs to a decoration } \\ \nu\left(\theta_{e}\right)=\cot \left(\frac{\theta_{e}}{2}\right) & \text { if } e \text { comes from an edge of } G\end{cases}
$$

We refer to these weights as critical dimer weights, and to the corresponding dimer model as critical dimer model on the Fisher graph $\mathcal{G}$.

\section{Critical characteristic polynomials}

In this section we define the critical dimer and Laplacian characteristic polynomials. We then state Forman's theorem proving that the Laplacian characteristic polynomial is a generating function for CRSFs of the underlying graph.

\subsection{Critical dimer characteristic polynomial}

The dimer model has the specific feature of having an explicit formula for the partition function due to Kasteleyn [Kas61] and independently to Temperley and Fisher [TF61]. It involves a weighted adjacency matrix of the underlying graph known as a Kasteleyn matrix. Let us define it for the critical dimer model on a Fisher graph $\mathcal{G}$, which we assume to be the Fisher graph of an infinite, $\mathbb{Z}^{2}$-periodic isoradial graph $G$. Recall that $\mathcal{G}_{1}=\mathcal{G} / \mathbb{Z}^{2}$ denotes the fundamental domain of $\mathcal{G}$.

A Kasteleyn orientation of $\mathcal{G}$ is an orientation of the edges of $\mathcal{G}$ such that all elementary cycles are clockwise odd, i.e. when traveling clockwise around the edges of any elementary cycle of $\mathcal{G}$, the number of co-oriented edges is odd. When the graph is planar, such an orientation always exists [Kas67]. For later purposes, we need to keep track of the orientation of the edges of $\mathcal{G}$. We thus choose a specific Kasteleyn orientation of $\mathcal{G}$ in which every triangle of every decoration is oriented clockwise. Having a Kasteleyn orientation of the graph $\mathcal{G}$ then amounts to finding a Kasteleyn orientation of the planar graph obtained from $\mathcal{G}$ by contracting each triangle to a single vertex, which exists by Kasteleyn's theorem [Kas67]. Refer to Figure 4 for an example of such an orientation in the case where $G=\mathbb{Z}^{2}$.

The Kasteleyn matrix corresponding to such an orientation is an infinite matrix, whose rows and columns are indexed by vertices of $\mathcal{G}$, defined by:

$$
K_{x, y}=\varepsilon_{x, y} \nu_{x y}
$$




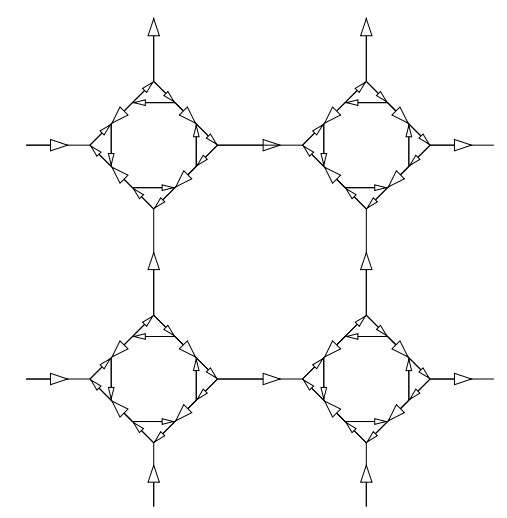

Figure 4: An example of Kasteleyn orientation of the Fisher graph of $\mathbb{Z}^{2}$, in which every triangle of every decoration is oriented clockwise.

where

$$
\varepsilon_{x, y}= \begin{cases}1 & \text { if } x \sim y, \text { and } x \rightarrow y \\ -1 & \text { if } x \sim y, \text { and } x \leftarrow y \\ 0 & \text { else },\end{cases}
$$

and $\nu$ is the critical dimer model weight function of Equation (1). Note that $K$ can be interpreted as an operator acting on $\mathbb{C}^{V(\mathcal{G})}$ :

$$
\forall f \in \mathbb{C}^{V(\mathcal{G})}, \quad(K f)_{x}=\sum_{x \in V(\mathcal{G})} K_{x, y} f_{y}
$$

The critical dimer characteristic polynomial, denoted by $P_{\text {dimer }}(\mathrm{z}, \mathrm{w})$, is the determinant of the Fourier transform of the Kasteleyn operator. More explicitly, let $\gamma_{\mathrm{h}}$ and $\gamma_{\mathrm{v}}$ be two paths in the dual graph of $\mathcal{G}_{1}$ winding once around the torus horizontally and vertically respectively. Then, the Fourier transform of $K$ is the modified weight Kasteleyn matrix $K_{1}(\mathrm{z}, \mathrm{w})$ whose lines and columns are indexed by vertices of $\mathcal{G}_{1}$, and whose coefficients are those of $K$ multiplied by $\mathrm{z}^{ \pm 1}$ (resp. $\mathrm{w}^{ \pm 1}$ ) when the corresponding edge is crossed by the horizontal cycle (resp. vertical cycle), and the sign \pm 1 is defined by the Kasteleyn orientation of the edge. The critical dimer characteristic polynomial then is:

$$
P_{\text {dimer }}(\mathrm{z}, \mathrm{w})=\operatorname{det} K_{1}(\mathrm{z}, \mathrm{w}) \text {. }
$$

It is the key ingredient used in explicit formulae for the critical dimer model defined on the infinite graph $\mathcal{G}$ or on the finite toroidal graph $\mathcal{G}_{n}=\mathcal{G} / n \mathbb{Z}^{2}, n \in \mathbb{N}^{*}$, see [BdT10b]. More precisely, the partition function of the fundamental domain $\mathcal{G}_{1}$ can be expressed as a linear combination of the square root of $P_{\text {dimer }}(z, w)$ evaluated at $z, w \in\{ \pm 1\}$, and the partition function of $\mathcal{G}_{n}$ can be expressed using $P_{\text {dimer }}(\mathrm{z}, \mathrm{w})$ evaluated at $\mathrm{z}^{n}, \mathrm{w}^{n} \in\{ \pm 1\}$. 
By expanding $P_{\text {dimer }}(\mathrm{z}, \mathrm{w})$, see also the proof of Lemma 13 of [BdT10b],

$$
P_{\text {dimer }}(\mathrm{z}, \mathrm{w})=\sum_{\sigma \in \mathcal{S}_{\left|V\left(\mathcal{G}_{1}\right)\right|}} \operatorname{sgn}(\sigma) \prod_{x \in V\left(\mathcal{G}_{1}\right)}\left(K_{1}(\mathrm{z}, \mathrm{w})\right)_{x, \sigma(x)},
$$

and using the fact that $K_{1}(\mathrm{z}, \mathrm{w})$ is an adjacency matrix, one observes that the only contribution to the sum comes from configurations which are unions of disjoint cycles covering all vertices of $\mathcal{G}_{1}$, such that trivial cycles (homotopic to a point) are of even length (when the length is 2, it is then a doubled edge), non-trivial cycles (with nontrivial homology) can be of even or odd length, and each non-trivial cycle contributes a term $z^{h} \mathbf{w}^{v}$ where $(h, v)$ is its homology class. Moreover, since cycles are disjoint, nontrivial cycles must be parallel. The difference between these configurations and superimposition of dimer configurations, also known as double-dimers, lies in the terms $\mathrm{z}, \mathrm{w}$ and in the fact that non-trivial cycles can be of odd length; double-dimer configurations can be recovered by taking a linear combination of $P_{\text {dimer }}(\mathrm{z}, \mathrm{w})$ with $\mathrm{z}, \mathrm{w} \in\{ \pm 1\}$. We refer to configurations counted by $P_{\text {dimer }}(\mathrm{z}, \mathrm{w})$ as 'double-dimer' configurations.

\subsection{Critical Laplacian characteristic polynomial}

A generalization of Kirchhoff's matrix tree theorem due to Forman [For93] proves that the Laplacian characteristic polynomial is a generating function for cycle rooted spanning forests, which are the natural pendent of spanning trees when working on the torus. In this section we first define cycle rooted spanning forests, then the Laplacian characteristic polynomial, and finally state Forman's theorem.

We let $G$ be an infinite, $\mathbb{Z}^{2}$-periodic isoradial graph, and $G_{1}=G / \mathbb{Z}^{2}$ be the fundamental domain. Note that the content of Sections 3.2.1 and 3.2.2 holds in more generality, i.e. when $G_{1}$ is any graph embedded on the torus and $\rho$ (see below) is any positive weight function on unoriented edges of $G_{1}$.

\subsubsection{Cycle rooted spanning forests}

A cycle-rooted tree (CRT) of a toroidal graph $G_{1}$ is a connected subgraph of $G_{1}$ with a unique non-trivial cycle. A cycle-rooted spanning forest (CRSF) is a collection of disjoint cycle-rooted trees covering every vertex of $G_{1}$, thus implying that all nontrivial cycles are parallel. An oriented CRT (OCRT) is a CRT in which edges of the branches are oriented towards the non-trivial cycle, and the non-trivial cycle is oriented in one of the two possible ways. An oriented CRSF (OCRSF) is a CRSF consisting of OCRTs.

Let us denote by $T$ a generic OCRT of $G_{1}$, by $F$ a generic oriented OCRSF of $G_{1}$, and by $\mathcal{F}\left(G_{1}\right)$ the collection of OCRSFs of $G_{1}$.

Remark 2. 


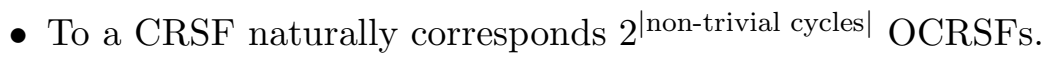

- A CRSF is characterized as a subset of $\left|V\left(G_{1}\right)\right|$ edges of $G_{1}$ containing no trivial cycle.

- An OCRSF is characterized as a subset of oriented edges of $G_{1}$ such that each vertex has exactly one outgoing edge of this subset, and which contains no trivial cycle.

Let $\gamma_{\mathrm{h}}$ and $\gamma_{\mathrm{v}}$ be two paths in the dual graph of $G_{1}$ winding once around the torus horizontally and vertically respectively. Assume that $\gamma_{\mathrm{h}}$ and $\gamma_{\mathrm{v}}$ are assigned a reference orientation. The homology class of an OCRT $T$, denoted by $H(T)=(h(T), v(T))$, is defined to be the homology class of its non-trivial cycle in $\mathbb{Z}^{2}$. Define the reference number of $T$ to be:

$$
H_{0}(T)=\left(h_{0}(T), v_{0}(T)\right)= \pm(h(T), v(T)),
$$

where the sign is chosen so that, $h_{0} \geq 0$, and $v_{0} \geq 0$ when $h_{0}=0$. Note that this definition is independent of the orientation of the non-trivial cycle, so that it also makes sense for CRTs. Define the sign of the non-trivial cycle of $T$ to be:

$$
N(T)=\mathbf{1}_{\left\{H(T)=H_{0}(T)\right\}}-\mathbf{1}_{\left\{H(T)=-H_{0}(T)\right\}} \cdot
$$

Then, the homology class of the OCRT $T$ can be rewritten as:

$$
H(T)=N(T) H_{0}(T)
$$

Let $F$ be an OCRSF of $G_{1}$, and denote by $T_{1}, \cdots, T_{n}$, its tree components, then the homology class $H(F)$ of $F$, is naturally defined by:

$$
H(F)=(h(F), v(F))=\sum_{i=1}^{n} H\left(T_{i}\right)=\left(\sum_{i=1}^{n} h\left(T_{i}\right), \sum_{i=1}^{n} v\left(T_{i}\right)\right) .
$$

Since non-trivial cycles of the CRT components of $F$ are parallel, we deduce that the number $H_{0}\left(T_{i}\right), i \in\{1, \cdots, n\}$, is independent of $i$. It is then natural to define the reference number of the OCRSF $F$ by:

$$
H_{0}(F)=\left(h_{0}(F), v_{0}(F)\right)=H_{0}\left(T_{i}\right)
$$

As a consequence, the homology class of the OCRSF $F$ can be rewritten as:

$$
H(F)=N(F) H_{0}(F),
$$

where $N(F)=\sum_{i=1}^{n} N\left(T_{i}\right)$ is the signed number of cycles of the OCRSF $F$. 


\subsubsection{Critical Laplacian characteristic polynomial}

Suppose that (unoriented) edges of $G$ are assigned Kenyon's critical weight function for the Laplacian [Ken02], denoted by $\rho$,

$$
\forall e \in E(G), \rho_{e}=\rho\left(\theta_{e}\right)=\tan \theta_{e}
$$

where $\theta_{e}$ is the rhombus half-angle of the edge $e$. Then, the critical Laplacian $\Delta$ on $G$, is represented by the following matrix, also denoted $\Delta$, whose lines and columns are indexed by vertices of $G$ :

$$
\Delta_{x, y}= \begin{cases}\rho_{x y} & \text { if } x \sim y \\ -\sum_{y \sim x} \rho_{x y} & \text { if } x=y \\ 0 & \text { otherwise }\end{cases}
$$

The critical Laplacian characteristic polynomial, $P_{\text {Lap }}(\mathrm{z}, \mathrm{w})$, is the determinant of the Fourier transform of the Laplacian operator, that is, $P_{\text {Lap }}(\mathrm{z}, \mathrm{w})=\operatorname{det} \Delta_{1}(\mathrm{z}, \mathrm{w})$, where $\Delta_{1}(\mathrm{z}, \mathrm{w})$ is the modified weight Laplacian matrix defined in a way similar to the modified weight Kasteleyn matrix.

A remarkable fact due to Kirchhoff is that, when the graph is finite and embedded in the plane, the absolute value of any cofactor of the Laplacian matrix yields the weighted number of spanning trees. Forman generalized this result, and for the case of the torus, his result can be stated as, see also Lemma 9 of [BdT10b]:

Theorem 3 ([For93]). The critical Laplacian characteristic polynomial is the following combinatorial sum:

$$
P_{\text {Lap }}(\mathrm{z}, \mathrm{w})=\sum_{F \in \mathcal{F}\left(G_{1}\right)}\left(\prod_{e=(x, y) \in F} \rho_{x y}\right) \prod_{T \in F}\left(1-\mathrm{z}^{h(T)} \mathrm{w}^{v(T)}\right) .
$$

Note that the weight function $\rho$ is independent of the orientation of the edges.

\section{Matrix-tree theorem for the Kasteleyn matrix}

Consider a critical Ising model defined on an infinite, $\mathbb{Z}^{2}$-periodic isoradial graph $G$, and let $\mathcal{G}$ be the Fisher graph of the corresponding critical dimer model. In Section 4.2, we state and prove Theorem 7 , which is a matrix-tree type theorem for the Kasteleyn matrix, thus establishing that the critical dimer characteristic polynomial can be rewritten as a generating function for OCRSFs of $\mathcal{G}_{1}$. In Section 4.3, we analyze this polynomial and show that the contribution of some OCRSFs cancel out, leading to the definition of essential OCRSFs of $\mathcal{G}_{1}$. Then, in Section 4.4, we give a precise statement of Theorem 1, and an idea of the proof. Section 4.1 is dedicated to notations. 


\subsection{Notations}

We specify notations and terminology for the isoradial graph $G$ and for the corresponding Fisher graph $\mathcal{G}$, which were introduced in [BdT10a], see also Figure 5. These will be used throughout the remainder of the paper.

Edges of $\mathcal{G}$ corresponding to edges of $G$ are referred to as long edges, and edges of the decorations of $\mathcal{G}$ are referred to as short ones.

Vertices of the graph $G$ are written in boldface, and those of $\mathcal{G}$ with normal symbols. Edges and edge subsets of $G$ are also written in boldface. Let $x$ be a vertex of $\mathcal{G}$, then $x$ belongs to the decoration corresponding to a unique vertex $\mathbf{x}$ of $G$. We shall also denote by $\mathbf{x}$ the decoration in $\mathcal{G}$. Conversely, vertices of a decoration $\mathbf{x}$ of $\mathcal{G}$ are labeled as follows. Let $d_{\mathbf{x}}$ be the degree of the vertex $\mathbf{x}$ in $G$, then the decoration $\mathbf{x}$ of $\mathcal{G}$ consists of $d_{\mathbf{x}}$ triangles, labeled $t_{1}(\mathbf{x}), \cdots, t_{d_{\mathbf{x}}}(\mathbf{x})$ in counterclockwise order, and $d_{\mathbf{x}}$ inner edges. Vertices of the triangle $t_{k}(\mathbf{x})$ are labeled $v_{k}(\mathbf{x}), w_{k}(\mathbf{x}), z_{k}(\mathbf{x})$ in counterclockwise order, where $v_{k}(\mathbf{x})$ is the only vertex incident to a long edge. We also refer to the triangle $t_{k}(\mathbf{x})$ as the triangle of the vertex $v_{k}(\mathbf{x})$. Whenever no confusion occurs, we drop the argument $\mathbf{x}$ in the notations above.
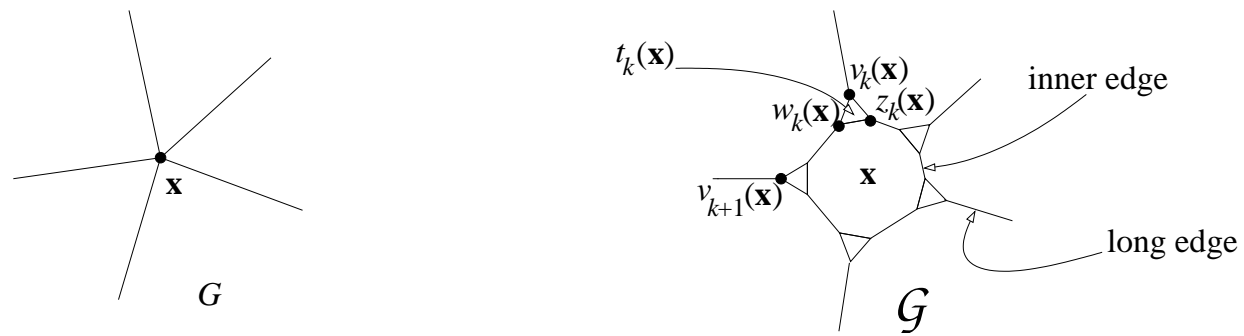

Figure 5: Left: vertex $\mathbf{x}$ of the graph $G$. Right: corresponding decoration $\mathbf{x}$ of $\mathcal{G}$.

Define a vertex $x$ of $\mathcal{G}$ to be of type ' $v$ ', if $x=v_{k}(\mathbf{x})$ for some vertex $\mathbf{x}$ of $G$ and some $k \in\left\{1, \cdots, d_{\mathbf{x}}\right\}$, and similarly for ' $w$ ' and ' $z$ '.

The isoradial embedding of the graph $G$ fixes an embedding of the corresponding diamond graph $G^{\diamond}$. There is a natural way of assigning rhombus unit-vectors of $G^{\diamond}$ to vertices of $\mathcal{G}$ : for every vertex $\mathbf{x}$ of $G$, and every $k \in\left\{1, \cdots, d_{\mathbf{x}}\right\}$, let us associate the rhombus unit-vector $e^{i \alpha_{w_{k}}(\mathbf{x})}$ to $w_{k}(\mathbf{x}), e^{i \alpha_{z_{k}}(\mathbf{x})}$ to $z_{k}(\mathbf{x})$, and the two rhombus-unit vectors $e^{i \alpha_{w_{k}}(\mathbf{x})}, e^{i \alpha_{z_{k}}(\mathbf{x})}$ to $v_{k}(\mathbf{x})$, as in Figure 6 below. Note that $e^{i \alpha_{w_{k}}(\mathbf{x})}=e^{i \alpha_{z_{k+1}}(\mathbf{x})}$.

\subsection{Matrix-tree theorem for the Kasteleyn matrix}

In this section, we prove a matrix-tree type theorem for the Kasteleyn matrix $K_{1}(\mathrm{z}, \mathrm{w})$ of the critical dimer model on the graph $\mathcal{G}_{1}$. A key requirement for such a theorem to hold is to have a vector in the kernel of the matrix $K$, which is the subject of the next section. 

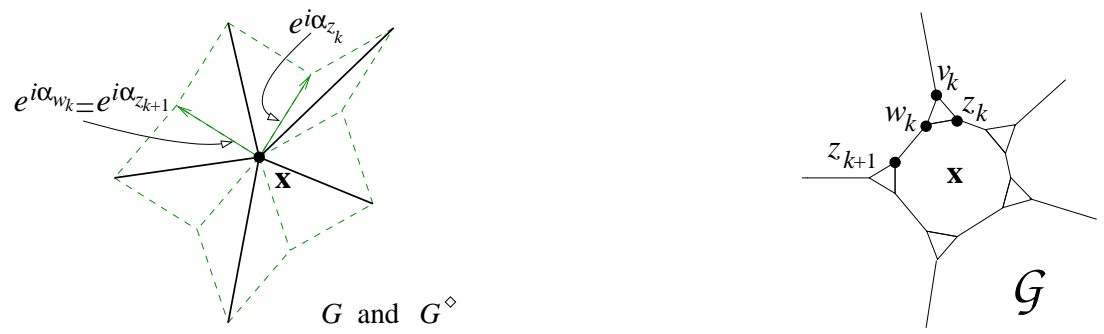

Figure 6: Rhombus vectors of the diamond graph $G^{\diamond}$ assigned to vertices of $\mathcal{G}$.

\subsubsection{Vector in the kernel of the Kasteleyn matrix}

The vector in the kernel of the Kasteleyn matrix $K$ is naturally obtained by setting $\lambda=0$ in the definition of the complex-valued function introduced in Section 4.2.2 of [BdT10a]. More precisely, we define $f=\left(f_{x}\right)_{\{x \in V(\mathcal{G})\}}$, by:

$$
f_{x}= \begin{cases}e^{-i \frac{\alpha_{w_{k}}(\mathbf{x})}{2}} & \text { if } x=w_{k}(\mathbf{x}) \\ -e^{-i \frac{\alpha_{z_{k}}(\mathbf{x})}{2}} & \text { if } x=z_{k}(\mathbf{x}) \\ f_{w_{k}(\mathbf{x})}+f_{z_{k}(\mathbf{x})} & \text { if } x=v_{k}(\mathbf{x}),\end{cases}
$$

for every vertex $\mathbf{x}$ of $G$, and every $k \in\left\{1, \cdots, d_{\mathbf{x}}\right\}$. Then, setting $\lambda=0$ in Proposition 15 of [BdT10a] yields:

Lemma 4. The vector $f$ is in the kernel of the matrix $K$. That is, if we let $x$ be a vertex of $\mathcal{G}$, and $x_{1}, x_{2}, x_{3}$ be its three neighbors, then:

$$
(K f)_{x}=\sum_{i=1}^{3} K_{x, x_{i}} f_{x_{i}}=0
$$

Remark 5. In order for the vector $f$ to be well defined, the angles $\alpha_{w_{k}(\mathbf{x})}, \alpha_{z_{k}(\mathbf{x})}$ need to be well defined mod $4 \pi$, indeed half-angles need to be well defined $\bmod 2 \pi$. The latter are defined inductively in [BdT10a] as follows, see also Figure 7. Note that the definition relies on our choice of Kasteleyn orientation of Section 3.1. Fix a vertex $\mathbf{x}_{0}$ of $G$, and set $\alpha_{z_{1}\left(\mathbf{x}_{0}\right)}=0$. Then, for vertices of $\mathcal{G}$ in the decoration of a vertex $\mathbf{x} \in G$, define:

$\alpha_{w_{k}(\mathbf{x})}=\alpha_{z_{k}(\mathbf{x})}+2 \theta_{k}(\mathbf{x})$, where $\theta_{k}(\mathbf{x})>0$ is the rhombus half-angle of Figure 7 ,

$\alpha_{z_{k+1}(\mathbf{x})}= \begin{cases}\alpha_{w_{k}(\mathbf{x})} & \text { if the edge } w_{k}(\mathbf{x}) z_{k+1}(\mathbf{x}) \text { is oriented from } w_{k}(\mathbf{x}) \text { to } z_{k+1}(\mathbf{x}) \\ \alpha_{w_{k}(\mathbf{x})}+2 \pi & \text { else. }\end{cases}$ 
Here is the rule defining angles in the neighboring decoration, corresponding to a vertex $\mathbf{y}$ of $G$. Let $k$ and $\ell$ be indices such that $v_{k}(\mathbf{x})$ is adjacent to $v_{\ell}(\mathbf{y})$ in $\mathcal{G}$. Then, define:

$$
\alpha_{w_{\ell}(\mathbf{y})}= \begin{cases}\alpha_{w_{k}(\mathbf{x})}-\pi & \text { if the edge } v_{k}(\mathbf{x}) v_{\ell}(\mathbf{y}) \text { is oriented from } v_{k}(\mathbf{x}) \text { to } v_{\ell}(\mathbf{y}) \\ \alpha_{w_{k}(\mathbf{x})}+\pi & \text { else. }\end{cases}
$$
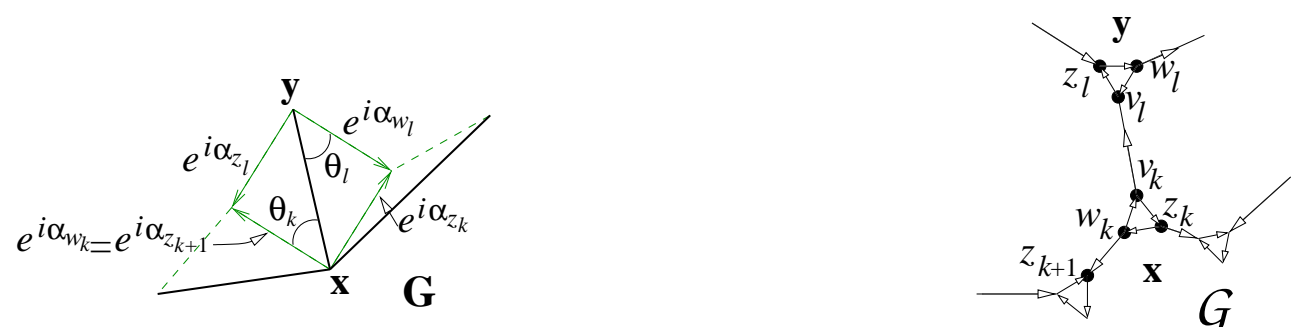

Figure 7: Notations for the definition of the angles in $\mathbb{R} / 4 \pi \mathbb{Z}$.

Lemma 6 ([BdT10a]). For every vertex $\mathbf{x}$ of $G$, and every $k \in\{1, \cdots, d(\mathbf{x})\}$, the angles $\alpha_{w_{k}(\mathbf{x})}, \alpha_{z_{k}(\mathbf{x})}$, are well defined in $\mathbb{R} / 4 \pi \mathbb{Z}$.

\subsubsection{Matrix-tree theorem}

The matrix-tree type theorem is most easily written for the matrix $K^{0}$, which is the following gauge transformation of the Kasteleyn matrix $K$ :

$$
K^{0}=D^{*} K D
$$

where $D$ is the diagonal matrix whose elements are indexed by vertices of $\mathcal{G}$, and such that $D_{x, x}=f_{x}$. Let $P_{\text {dimer }}^{0}(\mathrm{z}, \mathrm{w})=\operatorname{det} K_{1}^{0}(\mathrm{z}, \mathrm{w})$ be the characteristic polynomial of the matrix $K^{0}$, then we clearly have:

$$
P_{\text {dimer }}^{0}(\mathrm{z}, \mathrm{w})=\left(\prod_{x \in V\left(\mathcal{G}_{1}\right)}\left|f_{x}\right|^{2}\right) P_{\text {dimer }}(\mathrm{z}, \mathrm{w}) .
$$

Theorem 7. [Matrix-tree theorem for the Kasteleyn matrix]

The critical dimer characteristic polynomial $P_{\text {dimer }}^{0}(\mathrm{z}, \mathrm{w})$ is the following combinatorial sum:

$$
P_{\text {dimer }}^{0}(\mathrm{z}, \mathrm{w})=\sum_{F \in \mathcal{F}\left(\mathcal{G}_{1}\right)}\left(\prod_{e=(x, y) \in F} f_{x} \overline{f_{y}} K_{x, y}\right) \prod_{T \in F}\left(1-\mathrm{z}^{h(T)} \mathrm{w}^{v(T)}\right) .
$$


In order to prove Theorem 7, we introduce two modified incidence matrices $M$ and $N$ associated to the graph $\mathcal{G}$, defined as follows. Rows of $M$ are indexed by vertices of $\mathcal{G}$, columns by unoriented edges of $\mathcal{G}$, and:

$$
M_{x, e}= \begin{cases}K_{x, y} & \text { if the edge } e \text { is incident to } x, \text { and } e=x y \\ 0 & \text { if } e \text { is not incident to } x\end{cases}
$$

Rows of $N$ are indexed by unoriented edges of $\mathcal{G}$, columns by vertices of $\mathcal{G}$, and:

$$
N_{e, x}= \begin{cases}f_{x} \overline{f_{y}} & \text { if the edge } e \text { is incident to } x, \text { and } e=x y \\ 0 & \text { if } e \text { is not incident to } x\end{cases}
$$

The next lemma relates the Kasteleyn matrix $K^{0}$, and the incidence matrices $M$ and $N$.

Lemma 8. The following identity holds:

$$
K^{0}=M N
$$

Proof. Let $x$ and $y$ be two vertices of $\mathcal{G}$. If $x$ and $y$ are at distance more than 2 , then clearly $(M N)_{x, y}=0=K_{x, y}^{0}$. If $x$ and $y$ are neighbors, we have:

$$
(M N)_{x, y}=M_{x, e} N_{e, y}=K_{x, y} f_{y} \overline{f_{x}}=\left(D^{*} K D\right)_{x, y}=K_{x, y}^{0} .
$$

If $x=y$, then:

$$
\begin{aligned}
(M N)_{x, x} & =\sum_{e \text { incident to } x} M_{x, e} N_{e, x}=\sum_{y \sim x} K_{x, y} f_{x} \overline{f_{y}} \\
& =f_{x} \sum_{y \sim x} \overline{K_{x, y} f_{y}}, \quad \text { (since } K \text { is real) } \\
& =0, \quad(\text { by Lemma } 4) \\
& =K_{x, x}^{0} .
\end{aligned}
$$

Proof of Theorem 7. The proof is similar to that of the matrix-tree theorem on the torus, see [BdT10b] for example. Taking the Fourier transform of Equation (6) yields, $K_{1}^{0}(\mathrm{z}, \mathrm{w})=M_{1}\left(\mathrm{z}^{\frac{1}{2}}, \mathrm{w}^{\frac{1}{2}}\right) N_{1}\left(\mathrm{z}^{\frac{1}{2}}, \mathrm{w}^{\frac{1}{2}}\right)$, where $M_{1}(\mathrm{z}, \mathrm{w})$ and $N_{1}(\mathrm{z}, \mathrm{w})$ are the Fourier transform of the matrices $M$ and $N$. Moreover, since for $(\mathrm{z}, \mathrm{w}) \in \mathbb{T}^{2}$ the matrix $K_{1}^{0}(\mathrm{z}, \mathrm{w})$ is skew-hermitian, we have:

$$
P_{\text {dimer }}^{0}(\mathrm{z}, \mathrm{w})=\operatorname{det}\left(M_{1}\left(\mathrm{z}^{-\frac{1}{2}}, \mathrm{w}^{-\frac{1}{2}}\right) N_{1}\left(\mathrm{z}^{-\frac{1}{2}}, \mathrm{w}^{-\frac{1}{2}}\right)\right)
$$


where we also use the fact that $\left|V\left(\mathcal{G}_{1}\right)\right|=6\left|E\left(G_{1}\right)\right|$ is even. We now use Cauchy-Binet's formula to expand $P_{\text {dimer }}^{0}(\mathrm{z}, \mathrm{w})$ :

$$
P_{\text {dimer }}^{0}(\mathrm{z}, \mathrm{w})=\sum_{\substack{S \subset E\left(\mathcal{G}_{1}\right) \\|S|}} \operatorname{det}\left(M_{1}\left(\mathrm{z}^{-\frac{1}{2}}, \mathrm{w}^{-\frac{1}{2}}\right)^{S}\right) \operatorname{det}\left(N_{1}\left(\mathrm{z}^{-\frac{1}{2}}, \mathrm{w}^{-\frac{1}{2}}\right)_{S}\right) .
$$

Recall that $\gamma_{h}, \gamma_{v}$ are two paths in the dual graph of $\mathcal{G}_{1}$ winding once around the torus horizontally and vertically respectively. Suppose first that $S$ contains a trivial cycle, denoted by $C$, which does not cross the horizontal or the vertical cycle $\gamma_{\mathrm{h}}, \gamma_{\mathrm{v}}$. Let us show that in this case, $\operatorname{det}\left(M_{1}\left(\mathrm{z}^{-\frac{1}{2}}, \mathrm{w}^{-\frac{1}{2}}\right)^{S}\right)=0$. Define $\varphi: V\left(\mathcal{G}_{1}\right) \rightarrow \mathbb{C}$ by:

$$
\varphi_{x}= \begin{cases}1 & \text { if } x \in C \\ 0 & \text { else }\end{cases}
$$

Then, for every unoriented edge $e \in S$, we have:

$$
\begin{aligned}
& \left(\varphi^{t} M_{1}\left(\mathrm{z}^{-\frac{1}{2}}, \mathrm{w}^{-\frac{1}{2}}\right)^{S}\right)_{e}=\sum_{x \in V\left(\mathcal{G}_{1}\right)} \varphi_{x} M_{1}\left(\mathrm{z}^{-\frac{1}{2}}, \mathrm{w}^{-\frac{1}{2}}\right)_{x, e} \\
& \quad= \begin{cases}0 & \text { if } e \notin C \\
\varphi_{x} M_{1}\left(\mathrm{z}^{-\frac{1}{2}}, \mathrm{w}^{-\frac{1}{2}}\right)_{x, e}+\varphi_{y} M_{1}\left(\mathrm{z}^{-\frac{1}{2}}, \mathrm{w}^{-\frac{1}{2}}\right)_{y, e}=K_{x, y}+K_{y, x}=0 & \text { if } e=x y \in C .\end{cases}
\end{aligned}
$$

Thus, $\operatorname{det}\left(M_{1}\left(\mathrm{z}^{-\frac{1}{2}}, \mathrm{w}^{-\frac{1}{2}}\right)^{S}\right)=0$. If $C$ crosses the horizontal and/or vertical cycles $\gamma_{\mathrm{h}}$, $\gamma_{v}$, then the vector $\varphi$ in the kernel of $\left(M_{1}\left(\mathrm{z}^{-\frac{1}{2}}, \mathrm{w}^{-\frac{1}{2}}\right)^{S}\right)^{t}$ can be defined in a similar way. Therefore, the only contribution to the sum (7) comes from graphs whose number of edges equals the number of vertices and which contain no trivial cycle, i.e. from CRSFs.

Let us now compute the contribution of a CRSF $F$. After a possible reordering of the rows and columns of $M_{1}\left(\mathrm{z}^{-\frac{1}{2}}, \mathrm{w}^{-\frac{1}{2}}\right)$ and $N_{1}\left(\mathrm{z}^{-\frac{1}{2}}, \mathrm{w}^{-\frac{1}{2}}\right)$, we can suppose that both these matrices are block diagonal, each diagonal block corresponding to a connected component of $F$, i.e. a cycle rooted tree.

The determinant of a CRT $T$ in $M_{1}\left(\mathrm{z}^{-\frac{1}{2}}, \mathrm{w}^{-\frac{1}{2}}\right)\left(\right.$ resp. $\left.N_{1}\left(\mathrm{z}^{-\frac{1}{2}}, \mathrm{w}^{-\frac{1}{2}}\right)\right)$ can be evaluated by expanding it along columns (resp. rows) corresponding to leaves of the CRT. What remains then is the evaluation of the determinant reduced to the cycle. More precisely, suppose that edges of the branches are oriented from the leaves to the non-trivial cycle. Then, the contribution of the branches to $P_{\text {dimer }}^{0}(\mathrm{z}, \mathrm{w})$, is:

$$
\prod_{(x, y) \in \text { branch of } T} K_{x, y} f_{x} \overline{f_{y}} .
$$

Recall the definition of the reference number $H_{0}(T)=\left(h_{0}(T), v_{0}(T)\right)$ of the CRT $T$, given in Section 3.2.1, and let $x_{1}, \cdots, x_{n}$, be a labeling of the vertices of its non-trivial 
cycle in the direction given by $H_{0}(T)$. Then, the contribution of $T$ to $M_{1}\left(\mathrm{z}^{-\frac{1}{2}}, \mathrm{w}^{-\frac{1}{2}}\right)$ is:

$$
\left(\prod_{i=1}^{n} K_{x_{i}, x_{i+1}}\right) \mathrm{z}^{-\frac{h_{0}(T)}{2}} \mathrm{w}^{-\frac{v_{0}(T)}{2}}+(-1)^{n+1}\left(\prod_{i=1}^{n} K_{x_{i+1}, x_{i}}\right) \mathrm{z}^{\frac{h_{0}(T)}{2}} \mathrm{w}^{\frac{v_{0}(T)}{2}},
$$

and the contribution to $N_{1}\left(\mathrm{z}^{-\frac{1}{2}}, \mathrm{w}^{-\frac{1}{2}}\right)$ is:

$$
\left(\prod_{i=1}^{n} f_{x_{i}} \overline{f_{x_{i+1}}}\right) \mathrm{z}^{\frac{h_{0}(T)}{2}} \mathrm{w}^{\frac{v_{0}(T)}{2}}+(-1)^{n+1}\left(\prod_{i=1}^{n} \overline{f_{x_{i}}} f_{x_{i+1}}\right) \mathrm{z}^{-\frac{h_{0}(T)}{2}} \mathrm{w}^{-\frac{v_{0}(T)}{2}} .
$$

Since the Kasteleyn matrix $K$ is skew-symmetric, we have $(-1)^{n+1} \prod_{i=1}^{n} K_{x_{i+1}, x_{i}}=$ $-\prod_{i=1}^{n} K_{x_{i}, x_{i+1}}$, thus the contribution of the CRT $T$ is:

$$
\left(\prod_{i=1}^{n} f_{x_{i}} \overline{f_{x_{i+1}}} K_{x_{i}, x_{i+1}}\right)\left(1-\mathrm{z}^{h_{0}(T)} \mathrm{w}^{v_{0}(T)}\right)+\left(\prod_{i=1}^{n} f_{x_{i+1}} \overline{f_{x_{i}}} K_{x_{i+1}, x_{i}}\right)\left(1-\mathrm{z}^{-h_{0}(T)} \mathrm{w}^{-v_{0}(T)}\right) .
$$

Let $T_{1}$ and $T_{2}$ be the two OCRTs corresponding to $T$, then the contribution of the CRT $T$ can be rewritten as:

$$
\sum_{i=1}^{2}\left(\prod_{e=(x, y) \in T_{i}} f_{x} \overline{f_{y}} K_{x, y}\right)\left(1-\mathrm{z}^{h\left(T_{i}\right)} \mathrm{w}^{v\left(T_{i}\right)}\right) .
$$

Taking the product over all CRTs of the CRSF F, and summing over all CRSFs yields:

$$
\begin{aligned}
P_{\text {dimer }}^{0}(\mathrm{z}, \mathrm{w}) & =\sum_{F \in\left\{\mathrm{CRSF} \text { of } \mathcal{G}_{1}\right\}} \prod_{T \in\{\mathrm{CRT} \text { of } \mathrm{F}\}} \sum_{i=1}^{2}\left(\prod_{e=(x, y) \in T_{i}} f_{x} \overline{f_{y}} K_{x, y}\right)\left(1-\mathrm{z}^{h\left(T_{i}\right)} \mathrm{w}^{v\left(T_{i}\right)}\right), \\
& =\sum_{F \in \mathcal{F}\left(\mathcal{G}_{1}\right)} \prod_{T \in F}\left(\prod_{e=(x, y) \in T} f_{x} \overline{f_{y}} K_{x, y}\right)\left(1-\mathrm{z}^{h(T)} \mathrm{w}^{v(T)}\right) .
\end{aligned}
$$

\subsection{Characterization of OCRSFs contributing to $P_{\text {dimer }}^{0}(\mathrm{z}, \mathrm{w})$}

In this section, we characterize OCRSFs of $\mathcal{G}_{1}$ which contribute to $P_{\text {dimer }}^{0}(\mathrm{z}, \mathrm{w})$. Indeed, it turns out that the contributions of some of them cancel out.

More precisely, let $\mathbf{L}$ be a subset of oriented edges of $G_{1}$, defining a subset of oriented long edges $\mathbf{L}$ of $\mathcal{G}_{1}$. An OCRSF $F$ of $\mathcal{G}_{1}$ is said to be compatible with $\mathbf{L}$, if the long edges of $F$ are exactly those of $\mathbf{L}$. We first characterize OCRSFs compatible with $\mathbf{L}$, and then OCRSFs compatible with $\mathbf{L}$ which actually contribute to $P_{\text {dimer }}^{0}(z, w)$. 


\subsubsection{OCRSFs compatible with $\mathrm{L}$}

An oriented edge $(\mathbf{x}, \mathbf{y})$ of $\mathbf{L}$ corresponds to a unique oriented edge $\left(v_{k}(\mathbf{x}), v_{\ell}(\mathbf{y})\right)$ of $\mathcal{G}_{1}$. We refer to the vertex $v_{k}(\mathbf{x})$ as a root vertex of $\mathbf{L}$. This defines for every decoration $\mathbf{x}$, a set of root vertices denoted by $R_{\mathbf{x}}(\mathbf{L})$, and a set of non-root vertices of type ' $v$ ', $R_{\mathbf{x}}(\mathbf{L})^{c}:=\left\{v_{1}(\mathbf{x}), \cdots, v_{d_{\mathbf{x}}}(\mathbf{x})\right\} \backslash R_{\mathbf{x}}(\mathbf{L})$, see Figure 8 below for an example.

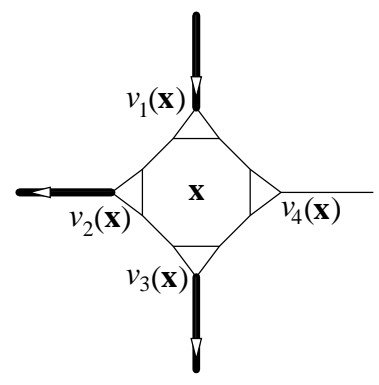

Figure 8: In this example $\mathbf{L}$ is drawn in bold lines, $R_{\mathbf{x}}(\mathbf{L})=\left\{v_{2}(\mathbf{x}), v_{3}(\mathbf{x})\right\}$ and $R_{\mathbf{x}}(\mathbf{L})^{c}=\left\{v_{1}(\mathbf{x}), v_{4}(\mathbf{x})\right\}$.

Remark 9. Suppose that one of the decoration $\mathbf{x}$ of $\mathcal{G}_{1}$ contains no root vertex of $\mathbf{L}$, and suppose that there is an OCRSF $F$ of $\mathcal{G}_{1}$ compatible with $\mathbf{L}$. Then, since by Remark 2, the OCRSF $F$ has exactly one outgoing edge at every vertex, this implies that $F$ restricted to the decoration $\mathbf{x}$ has a number of edges equal to the number of vertices of the decoration, thus it must contain a trivial cycle and cannot be an OCRSF. As a consequence, we only consider subset of oriented edges $\mathbf{L}$ of $G_{1}$ such that:

Every decoration of $\mathcal{G}_{1}$ has at least one root vertex of $\mathbf{L}$.

Lemma 10. A subset of oriented edges $F$ of $\mathcal{G}_{1}$ is an OCRSF compatible with $\mathbf{L}$, iff the following conditions hold:

1. long edges of $F$ are exactly those of $\mathbf{L}$,

2. F contains no cycle consisting of long and short edges,

3. the restriction of $F$ to every decoration $\mathbf{x}$, is an oriented spanning forest with $\left|R_{\mathbf{x}}(\mathbf{L})\right|$ connected components whose roots are distinct elements of $R_{\mathbf{x}}(\mathbf{L})$.

Remark 11. Condition 3. is equivalent to Condition $3^{\prime}$ : the restriction of $F$ to every decoration $\mathbf{x}$ contains no trivial cycle, and is such that vertices of $R_{\mathbf{x}}(\mathbf{L})$ have no outgoing edge of $F$, and every other vertex has exactly one outgoing edge of $F$.

Proof. Let $F$ be a subset of oriented edges of $\mathcal{G}_{1}$. Then by the geometry of the graph $\mathcal{G}_{1}, F$ cannot have trivial cycles consisting of long edges only. Thus, Conditions $1,2,3^{\prime}$ 
are equivalent to saying that the oriented edge configuration $F$ is compatible with $\mathbf{L}$, contains no trivial cycle, and is such that every vertex of $\mathcal{G}_{1}$ has exactly one outgoing edge of $F$. By Remark 2, this is equivalent to saying that $F$ is an OCRSF compatible with $\mathbf{L}$.

\subsubsection{Restriction to decorations of OCRSFs contributing to $P_{\text {dimer }}^{0}(\mathrm{z}, \mathrm{w})$}

In this section, we characterize the restriction to decorations of OCRSFs of $\mathcal{G}_{1}$ compatible with $\mathbf{L}$, which contribute to $P_{\text {dimer }}^{0}(z, w)$. To this purpose we first introduce the following definition, see also Figure 9.

Definition 4.1. A subset of oriented edges of the decoration $\mathbf{x}$, is called an essential configuration compatible with $\mathbf{L}$, of type $\mathrm{cw}$ (resp. cclw), if it consists of:

1. all inner edges oriented clockwise (resp. counterclockwise).

2. one of the three following 2-edge configurations at the triangle of every non-root vertex $v_{i} \in R_{\mathbf{x}}(\mathbf{L})^{c}$.

$$
\begin{aligned}
& -\left\{\left(w_{i}, v_{i}\right),\left(v_{i}, z_{i}\right)\right\},\left\{\left(w_{i}, z_{i}\right),\left(v_{i}, z_{i}\right)\right\},\left\{\left(v_{i}, w_{i}\right),\left(w_{i}, z_{i}\right)\right\} \text { in the } c w \text { case } \\
& -\left\{\left(z_{i}, v_{i}\right),\left(v_{i}, w_{i}\right)\right\},\left\{\left(v_{i}, z_{i}\right),\left(z_{i}, w_{i}\right)\right\},\left\{\left(z_{i}, w_{i}\right),\left(v_{i}, w_{i}\right)\right\} \text { in the cclw case }
\end{aligned}
$$

3. one of the two following 1-edge configurations at the triangle of every root vertex $v_{i} \in R_{\mathbf{x}}(\mathbf{L})$ :

$$
\begin{aligned}
& -\left\{\left(w_{i}, v_{i}\right)\right\},\left\{\left(w_{i}, z_{i}\right)\right\} \text { in the } c w \text { case } \\
& -\left\{\left(z_{i}, v_{i}\right),\left\{\left(z_{i}, w_{i}\right)\right\}\right. \text { in the cclw case }
\end{aligned}
$$

with the additional constraint that the triangle of at least one root vertex contains the configuration $\left(w_{i}, v_{i}\right)$ in the $c w$ case, and the configuration $\left(z_{i}, v_{i}\right)$ in the $c c l w$ case.

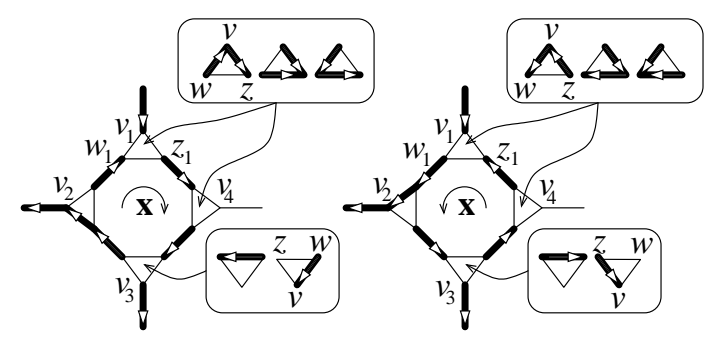

Figure 9: Essential configuration of the decoration $\mathbf{x}$ of type $c w$ (left) and of type $c c l w$ (right). 
When the type is not specified, we refer to the above as an essential configuration compatible with $\mathbf{L}$, and when no confusion occurs, we omit the specification compatible with $\mathbf{L}$.

\section{Proposition 12.}

1. For every decoration $\mathbf{x}$, an essential configuration compatible with $\mathbf{L}$ is an oriented spanning forest with $\left|R_{\mathbf{x}}(\mathbf{L})\right|$ connected components whose roots are distinct elements of $R_{\mathbf{x}}(\mathbf{L})$.

2. Let $F$ be an OCRSF of $\mathcal{G}_{1}$ compatible with $\mathbf{L}$, which contributes to $P_{\text {dimer }}^{0}(\mathrm{z}, \mathrm{w})$, then the restriction of $F$ to every decoration $\mathbf{x}$, is an essential configuration compatible with $\mathbf{L}$.

Proof. Proposition 12 is a direct consequence of the next two lemmas.

Lemma 13. Let $F$ be an OCRSF of $\mathcal{G}_{1}$ compatible with $\mathbf{L}$, which contributes to $P_{\text {dimer }}^{0}(\mathrm{z}, \mathrm{w})$. Then the restriction of $F$ to every decoration $\mathbf{x}$ contains all inner edges of the decoration, one edge at triangles of root vertices, and two edges at triangles of non-root vertices.

Proof. Let $F$ be an OCRSF of $\mathcal{G}_{1}$ compatible with $\mathbf{L}$. By Lemma 10, the restriction of $F$ to every decoration $\mathbf{x}$, is an oriented spanning forest with $\left|R_{\mathbf{x}}(\mathbf{L})\right|$ components, whose roots are distinct elements of $R_{\mathbf{x}}(\mathbf{L})$. This implies that:

a. Triangles of decorations contain at most 2 edges of $F$.

$b$. Each vertex of $\mathcal{G}_{1}$ has a unique outgoing edge of $F$, and each root vertex of $R_{\mathbf{x}}(\mathbf{L})$ has as outgoing edge of $F$ the unique incident long edge (which belongs to $\mathbf{L}$ by definition of root vertices).

c. The restriction of $F$ to every decoration $\mathbf{x}$ contains $3 d_{\mathbf{x}}-\left|R_{\mathbf{x}}(\mathbf{L})\right|$ edges.

Let us first prove that the contribution to $P_{\text {dimer }}^{0}(\mathrm{z}, \mathrm{w})$ of OCRSFs having two edges at triangles of root vertices cancel out. Let $\mathbf{x}$ be a decoration of $\mathcal{G}_{1}$, and $v_{i} \in R_{\mathbf{x}}(\mathbf{L})$ be a root vertex of $\mathbf{x}$. Then, there are three possible oriented 2-edge configurations at the triangle $t_{i}$ of the vertex $v_{i}$, which satisfy the necessary requirement of Point $b$. above, see Figure 10:

$$
T_{1}=\left\{\left(w_{i}, v_{i}\right),\left(z_{i}, v_{i}\right)\right\}, \quad T_{2}=\left\{\left(w_{i}, z_{i}\right),\left(z_{i}, v_{i}\right)\right\}, \quad T_{3}=\left\{\left(z_{i}, w_{i}\right),\left(w_{i}, v_{i}\right)\right\} .
$$

Suppose that there exists an OCRSF $F^{1}$ compatible with $\mathbf{L}$, having the edge configuration $T_{1}$ at the triangle $t_{i}$. Let $F^{2}$ (resp. $F^{3}$ ) be the OCRSF $F^{1}$ modified so as to have the edge configuration $T_{2}$ (resp. $T_{3}$ ) at the triangle $t_{i}$. Then, it is straightforward to check that $F^{1}, F^{2}$ and $F^{3}$ are OCRSFs compatible with $\mathbf{L}$, with oriented non-trivial 


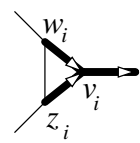

$T_{1}$

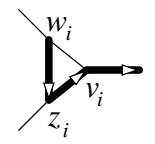

$T_{2}$

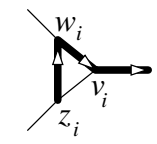

$T_{3}$

Figure 10: The three possible oriented 2-edge configurations at triangles of root vertices.

cycles in bijection (one way to prove this is to use Part 1 of Lemma 20). By Theorem 7, the contribution $C\left(F^{1}, F^{2}, F^{3}\right)$ of $F^{1}, F^{2}, F^{3}$ to $P_{\text {dimer }}^{0}(\mathrm{z}, \mathrm{w})$ is:

$$
C\left(F^{1}, F^{2}, F^{3}\right):=\sum_{j=1}^{3}\left(\prod_{e=(x, y) \in F^{j}} f_{x} \overline{f_{y}} K_{x, y}\right) \prod_{T \in F^{j}}\left(1-z^{h(T)} w^{v(T)}\right) .
$$

Since $F^{1}, F^{2}, F^{3}$ have oriented non-trivial cycles in bijection, the term $\prod_{T \in F^{j}}(1-$ $\left.z^{h(T)} w^{v(T)}\right)$ is independent of $j$. Denote by $C$ the contribution of edges of $F^{1}$ which are not edges of the triangle $t_{i}$. Then:

$$
\begin{aligned}
& C\left(F^{1}, F^{2}, F^{3}\right)=C \prod_{T \in F^{1}}\left(1-z^{h(T)} w^{v(T)}\right) . \\
& \quad \cdot\left(f_{w_{i}} \overline{f_{v_{i}}} K_{w_{i}, v_{i}} f_{z_{i}} \overline{f_{v_{i}}} K_{z_{i}, v_{i}}+f_{w_{i}} \overline{f_{z_{i}}} K_{w_{i}, z_{i}} f_{z_{i}} \overline{f_{v_{i}}} K_{z_{i}, v_{i}}+f_{z_{i}} \overline{f_{w_{i}}} K_{z_{i}, w_{i}} f_{w_{i}} \overline{f_{v_{i}}} K_{w_{i}, v_{i}}\right) .
\end{aligned}
$$

Recalling that by our choice of Kasteleyn orientation, edges of the triangles are oriented clockwise, we have that $C\left(F^{1}, F^{2}, F^{3}\right)$ is equal to:

$$
\begin{aligned}
& C \prod_{T \in F^{1}}\left(1-z^{h(T)} w^{v(T)}\right)\left[f_{w_{i}} \overline{f_{v_{i}}} f_{z_{i}} \overline{f_{v_{i}}}(-1)+(-1) f_{w_{i}} \overline{f_{z_{i}}}(-1) f_{z_{i}} \overline{f_{v_{i}}}+f_{z_{i}} \overline{f_{w_{i}}} f_{w_{i}} \overline{f_{v_{i}}}\right] \\
& =C \prod_{T \in F^{1}}\left(1-z^{h(T)} w^{v(T)}\right)\left[f_{z_{i}} f_{w_{i}} \overline{f_{v_{i}}}\left(-\overline{f_{v_{i}}}+\overline{f_{z_{i}}}+\overline{f_{w_{i}}}\right)\right] \\
& =0 \quad \text { (by definition of the vector } f) .
\end{aligned}
$$

Since this holds for every decoration $\mathbf{x}$, and every root vertex $v_{i} \in R_{\mathbf{x}}(\mathbf{L})$, we deduce that the contribution to $P_{\text {dimer }}^{0}(\mathrm{z}, \mathrm{w})$ of OCRSFs compatible with $\mathbf{L}$, having two edges at triangles of root vertices, cancel out.

As a consequence, if an OCRSF compatible with $\mathbf{L}$ contributes to $P_{\text {dimer }}^{0}(\mathbf{z}, \mathbf{w})$, then the restriction of $F$ at the decoration $\mathbf{x}$ contains at most, all inner edges of the decoration, one edge at triangles of root vertices, and two edges at triangles of non-root vertices; that is it contains at most $d_{\mathbf{x}}+\left|R_{\mathbf{x}}(\mathbf{L})\right|+2\left|R_{\mathbf{x}}(\mathbf{L})^{c}\right|=3 d_{\mathbf{x}}-\left|R_{\mathbf{x}}(\mathbf{L})\right|$ edges. Since by Point $c$. above, it must contain exactly $3 d_{\mathbf{x}}-\left|R_{\mathbf{x}}(\mathbf{L})\right|$ edges, we deduce that all constraints must be met. 
Lemma 14. A subset of edges of the decoration $\mathbf{x}$, is an oriented spanning forest with $\left|R_{\mathbf{X}}(\mathbf{L})\right|$ components whose roots are distinct elements of $R_{\mathbf{x}}(\mathbf{L})$, satisfying the constraints of Lemma 13, iff it is an essential configuration compatible with $\mathbf{L}$.

Proof. By Remark 11, Lemma 14 amounts to proving that an oriented subset of the decoration $\mathbf{x}$ contains all inner edges of the decoration, one edge at triangles of root vertices, two edges at triangles of non-root vertices, and satisfies Condition $3^{\prime}$, iff it is an essential configuration compatible with $\mathbf{L}$. This is clear once we return to Definition 4.1.

\subsubsection{Essential OCRSFs of $\mathcal{G}_{1}$}

Sections 4.3.1 and 4.3.2 above naturally yield the following definitions.

Definition 4.2. Let $\tau \in\{c w, c c w l\}^{V\left(G_{1}\right)}$ be an assignment of type $c w$ or $c c l w$ to the vertices of $G_{1}$. Define the set of essential OCRSFs of $\mathcal{G}_{1}$ compatible with $\mathbf{L}$ and $\tau$, to be the set of OCRSFs compatible with $\mathbf{L}$ whose restriction to every decoration $\mathbf{x}$, is an essential configuration of type $\tau(\mathbf{x})$. We denote this set by $\mathcal{F}^{\tau, \mathbf{L}}\left(\mathcal{G}_{1}\right)$.

As a consequence of Lemma 10 and Proposition 12, we have the following characterization of $\mathcal{F}^{\tau, \mathbf{L}}\left(\mathcal{G}_{1}\right)$.

Corollary 15. A subset of oriented edges of $\mathcal{G}_{1}$ is an essential OCRSF compatible with $\mathbf{L}$ and $\tau$, iff:

1. long edges of $F$ are exactly those of $\mathbf{L}$,

2. F contains no trivial cycle consisting of short and long edges,

3. the restriction of $F$ to every decoration $\mathbf{x}$ of $\mathcal{G}_{1}$, is an essential configuration compatible with $\mathbf{L}$, of type $\tau(\mathbf{x})$.

Definition 4.3. Define the set of essential OCRSFs of $\mathcal{G}_{1}$ compatible with $\mathbf{L}$, denoted $\mathcal{F}^{\mathbf{L}}\left(\mathcal{G}_{1}\right)$, by:

$$
\mathcal{F}^{\mathbf{L}}\left(\mathcal{G}_{1}\right)=\bigcup_{\tau \in\{c w, c c l w\}^{V\left(G_{1}\right)}} \mathcal{F}^{\tau, \mathbf{L}}\left(\mathcal{G}_{1}\right),
$$

and the set of essential OCRSFs of $\mathcal{G}_{1}$, denoted $\mathcal{F}^{0}\left(\mathcal{G}_{1}\right)$, by:

$$
\mathcal{F}^{0}\left(\mathcal{G}_{1}\right)=\bigcup_{\mathbf{L} \in \mathcal{L}} \mathcal{F}^{\mathbf{L}}\left(\mathcal{G}_{1}\right)
$$

where $\mathcal{L}$ is the set of oriented edge configurations of $G_{1}$ satisfying (*). 


\section{Corollary 16.}

$$
P_{\text {dimer }}^{0}(\mathrm{z}, \mathrm{w})=\sum_{F \in \mathcal{F}^{0}\left(\mathcal{G}_{1}\right)}\left(\prod_{e=(x, y) \in F} f_{x} \overline{f_{y}} K_{x, y}\right) \prod_{T \in F}\left(1-\mathrm{z}^{h(T)} \mathrm{w}^{v(T)}\right) .
$$

Proof. Write, $\mathcal{F}\left(\mathcal{G}_{1}\right)=\mathcal{F}^{0}\left(\mathcal{G}_{1}\right) \cup\left(\mathcal{F}^{0}\left(\mathcal{G}_{1}\right)\right)^{c}$ in the formula for $P_{\text {dimer }}^{0}(\mathrm{z}, \mathrm{w})$ given by Theorem 7. Then use Point 2. of Proposition 12, to deduce that the contribution of OCRSFs of $\left(\mathcal{F}^{0}\left(\mathcal{G}_{1}\right)\right)^{c}$ cancel out in $P_{\text {dimer }}^{0}(\mathrm{z}, \mathrm{w})$.

\subsection{Statement of main result}

We can now give a precise statement of Theorem 1 .

Theorem 17. Consider a critical Ising model defined on an infinite, $\mathbb{Z}^{2}$-periodic isoradial graph $G$. Then, one can explicitly construct essential OCRSFs of $\mathcal{G}_{1}$ counted by the critical dimer characteristic polynomial $P_{\text {dimer }}^{0}(\mathrm{z}, \mathrm{w})$, from OCRSFs of $G_{1}$ counted by the critical Laplacian characteristic polynomial $P_{\mathrm{Lap}}(\mathrm{z}, \mathrm{w})$.

Let us give an idea of the construction. First observe that the number of OCRSFs of $\mathcal{G}_{1}$ is much greater than the number of OCRSFs of $G_{1}$ so that there is certainly no one-to-one mapping between these set of configurations. Rather, to every OCRSF of $G_{1}$, we assign a family of OCRSFs of $\mathcal{G}_{1}$, which have the same reference number and the the same signed number of cycles, and such that the sum of the weights of OCRSFs in this family is equal to the weight of the original OCRSF of $G_{1}$. The family of OCRSF of $\mathcal{G}_{1}$ is constructed by successively adding long edges, and this construction is done by induction on the number of long edges added, thus allowing us to keep control over properties of OCRSFs in this family. To be more precise, we actually need to work on the graph $G_{1}$ and its dual graph $G_{1}^{*}$ at the same time. The operations used to add long edges are called licit primal/dual moves, and are valid in a context more general than isoradial and Fisher graphs, so that we dedicate it Section 5. The proof of Theorem 17 is the subject of Section 6. In Section 6.1, we construct a family of CRSFs of $\mathcal{G}_{1}$ from each CRSF of $G_{1}$. In Theorem 29 we prove that we exactly obtain all CRSFs of $\mathcal{G}_{1}$ counted by the dimer characteristic polynomial; and in Section 6.2, we show that this construction is weight preserving.

\section{Primal/dual edge moves on pairs of dual OCRSFs}

Definitions and results of this section are valid in a context more general than isoradial and Fisher graphs. But, in order not to introduce too many notations, we let $G_{1}$ be any graph embedded in the torus. 
In Section 5.1, we first define a general OCRSF characteristic polynomial, allowing for weights which depend on oriented edges, and we prove a useful rewriting of this polynomial, using pairs of dual OCRSFs. In Section 5.3, we define and prove properties of licit primal/dual edge moves, which are edge moves performed on pairs of dual OCRSFs, and are one of the key ingredients of the construction of Theorem 17. Licit primal/dual edge moves rely on a natural edge operation performed on one OCRSF only, which is the subject of Section 5.2.

\subsection{OCRSF characteristic polynomial}

We use definitions and notations introduced in Section 3.2.1. Suppose that a complex weight function $\rho$ is assigned to oriented edges of $G_{1}$, that is every oriented edge $(x, y)$ has a weight $\rho_{(x, y)} \in \mathbb{C}$. The OCRSF characteristic polynomial, corresponding to the weight function $\rho$, denoted $P_{\mathrm{OCRSF}}(\mathrm{z}, \mathrm{w})$, is defined by:

$$
P_{\mathrm{OCRSF}}(\mathrm{z}, \mathrm{w})=\sum_{F \in \mathcal{F}\left(G_{1}\right)} \rho(F) \prod_{T \in F}\left(1-\mathrm{z}^{h(T)} \mathrm{w}^{v(T)}\right),
$$

where $\rho(F)=\prod_{(x, y) \in F} \rho_{(x, y)}$.

Remark 18. When $\rho$ is a positive weight function on unoriented edges of $G_{1}$, then the OCRSF characteristic polynomial is simply the Laplacian characteristic polynomial.

We now prove a useful rewriting of $P_{\mathrm{OCRSF}}(\mathrm{z}, \mathrm{w})$. In order to do this, we need a few more facts and definitions. Denote by $G_{1}^{*}$ the dual graph of $G_{1}$ and let us, for a moment, consider edge configurations as unoriented. It is a general fact that if $F$ is a CRSF of $G_{1}$, then the complementary of the dual edge configuration, consisting exactly of the dual edges of the edges absent in $F$, is a CRSF of $G_{1}^{*}$, with non trivial cycles parallel to those of $F$, such that primal and dual non trivial cycles alternate along the torus. It is referred to as the dual CRSF of $F$.

Let $F$ and $F^{*}$ be OCRSFs of $G_{1}$ and $G_{1}^{*}$ respectively, then $F$ and $F^{*}$ are said to be dual of eachother, if their unoriented versions are. This means that to a pair of dual CRSFs corresponds $4^{\text {non-trivial cycles| }}$ pairs of dual OCRSFs. We denote by $\mathcal{F}\left(G_{1}, G_{1}^{*}\right)$ the set of pairs of dual OCRSFs, that is:

$$
\mathcal{F}\left(G_{1}, G_{1}^{*}\right)=\left\{\left(F, F^{*}\right) \in \mathcal{F}\left(G_{1}\right) \times \mathcal{F}\left(G_{1}^{*}\right): F, F^{*} \text { are dual OCRSFs }\right\} .
$$

Let $\left(F, F^{*}\right)$ be a pair of dual OCRSFs of $G_{1}$ and $G_{1}^{*}$. Recall that the homology class $H(F)$ of $F$ can be rewritten as:

$$
H(F)=N(F) H_{0}(F),
$$

where $H_{0}(F)=\left(h_{0}(F), v_{0}(F)\right)$ is the reference number of $F$, and $N(F)$ is the signed number of non-trivial cycles of $F$ defined in Section 3.2.1; and similarly for the homology 
class of $F^{*}$. Observe that $F$ and $F^{*}$ have the same reference number, so that it makes sense to refer to $H_{0}(F)$ as the reference number of the pair $\left(F, F^{*}\right)$. Let us denote by $N\left(F, F^{*}\right)$ the sum $N(F)+N\left(F^{*}\right)$. Then, we have the following rewriting of $P_{\text {OCRSF }}$.

Lemma 19. The OCRSF characteristic polynomial of $G_{1}$ can be rewritten as:

$$
P_{\mathrm{OCRSF}}(\mathrm{z}, \mathrm{w})=\sum_{\left(F, F^{*}\right) \in \mathcal{F}\left(G_{1}, G_{1}^{*}\right)} \rho(F)\left(-\mathrm{z}^{h_{0}(F)} \mathrm{w}^{v_{0}(F)}\right)^{\frac{1}{2} N\left(F, F^{*}\right)} .
$$

Proof. Denote by $T_{1}, \cdots, T_{n}$ the OCRT components of a generic OCRSF $F$ of $G_{1}$. By expanding the product of Equation (8), the polynomial $P_{\text {OCRSF }}(z, w)$ can be rewritten as:

$$
\begin{aligned}
P_{\text {OCRSF }}(\mathbf{z}, \mathbf{w}) & =\sum_{F \in \mathcal{F}\left(G_{1}\right)} \rho(F) \prod_{i=1}^{n} \sum_{\varepsilon_{i} \in\{0,1\}}\left(-\mathrm{z}^{h\left(T_{i}\right)} \mathrm{w}^{v\left(T_{i}\right)}\right)^{\varepsilon_{i}} \\
& =\sum_{F \in \mathcal{F}\left(G_{1}\right)} \rho(F) \sum_{\left(\varepsilon_{1}, \cdots, \varepsilon_{n}\right) \in\{0,1\}^{n}} \prod_{i=1}^{n}\left(-\mathrm{z}^{h\left(T_{i}\right)} \mathrm{w}^{v\left(T_{i}\right)}\right)^{\varepsilon_{i}} .
\end{aligned}
$$

Fix an OCRSF $F$ of $G_{1}$, and let $\left(\varepsilon_{1}, \cdots, \varepsilon_{n}\right) \in\{0,1\}^{n}$. Then to $F$ assign the following dual OCRSF $F^{*}$ : if $\varepsilon_{i}=1$, take $T_{i}^{*}$ to be co-oriented with $T_{i}$, and if $\varepsilon_{i}=0$, take $T_{i}^{*}$ to be contra-oriented. Thus, there is a one-to-one correspondence between dual OCRSFs of $F$, and sequences $\left(\varepsilon_{1}, \cdots, \varepsilon_{n}\right) \in\{0,1\}^{n}$, and:

$$
\begin{aligned}
& \varepsilon_{i}= \pm \frac{1}{2}\left(N\left(T_{i}\right)+N\left(T_{i}^{*}\right)\right), \\
& \varepsilon_{i}\left(h\left(T_{i}\right), v\left(T_{i}\right)\right)=\frac{1}{2}\left(h\left(T_{i}\right)+h\left(T_{i}^{*}\right), v\left(T_{i}\right)+v\left(T_{i}^{*}\right)\right) .
\end{aligned}
$$

This implies that:

$$
\begin{aligned}
(-1)^{\varepsilon_{i}} & =(-1)^{\frac{1}{2}\left(N\left(T_{i}\right)+N\left(T_{i}^{*}\right)\right)}, \\
\varepsilon_{i}\left(h\left(T_{i}\right), v\left(T_{i}\right)\right) & =\frac{1}{2}\left(N\left(T_{i}\right)+N\left(T_{i}^{*}\right)\right)\left(h_{0}\left(T_{i}\right), v_{0}\left(T_{i}\right)\right) .
\end{aligned}
$$

The proof is concluded by recalling that $\sum_{i=1}^{n}\left(N\left(T_{i}\right), N\left(T_{i}^{*}\right)\right)=\left(N(F), N\left(F^{*}\right)\right)=$ $N\left(F, F^{*}\right)$ and that for every $i \in\{1, \cdots, n\}, H_{0}(F)=H_{0}\left(T_{i}\right)$.

\subsection{Replacing one edge of an OCRSF}

Let $F$ be an OCRSF of $G_{1}$, and consider an oriented edge $e=(x, y)$ of $E\left(G_{1}\right) \backslash F$. Then, since $F$ is an OCRSF, the vertex $x$ has a unique outgoing edge $e_{x}$ of $F$. Define $F_{\left\{e, e_{x}\right\}}$ to be the edge configuration $F$ where the edge $e_{x}$ is replaced by the edge $e$ :

$$
F_{\left\{e, e_{x}\right\}}:=F \cup\{e\} \backslash\left\{e_{x}\right\} .
$$


In this section we describe features of the edge configuration $F_{\left\{e, e_{x}\right\}}$, which by construction contains one outgoing edge at every vertex.

Denote by $T_{1}, \cdots, T_{n}$ the CRT components of $F$, and let $\gamma_{i}$ be the non-trivial cycle of the CRT component $T_{i}$. Without loss of generality let us suppose $n \geq 2$, the case $n=1$ simply being a boundary case of the case $n=2$. Then, the edge $e$ belongs to a unique cylinder $\mathscr{C}$ obtained by cutting along two neighboring non trivial cycles of $F$, say $\gamma_{1}, \gamma_{2}$, see Figures 11, 12, 13. Since $F$ is an OCRSF, the vertex $x$ (resp. $y$ ) is connected by an oriented edge-path $p_{x}$ (resp. $p_{y}$ ) of $F$ to one of the non-trivial cycles $\gamma_{1}$ or $\gamma_{2}$. Let $F^{*}$ be a dual OCRSF of $F$ (at this point the orientation of its non-trivial cycles does not matter), then there is a unique non-trivial cycle $\gamma^{*}$ of $F^{*}$ contained in the cylinder $\mathscr{C}$. Cutting along $\gamma^{*}$ separates the cylinder $\mathscr{C}$ into two disjoint cylinders $\mathscr{C}_{1}, \mathscr{C}_{2}$. In order to analyze the features of the edge configuration $F_{\left\{e, e_{x}\right\}}$, we first describe the edge configuration $F \cup\{e\}$, split according to the following cases.

Case 1: The edge $e$ belongs to the cylinder $\mathscr{C}_{1}$, see Figure 11 (the case where $e$ belongs to the cylinder $\mathscr{C}_{2}$ is symmetric). Then, the vertices $x$ and $y$ are connected to the path $\gamma_{1}$, and the paths $p_{x}$ and $p_{y}$ are contained in the cylinder $\mathscr{C}_{1}$. In Case 1 , we suppose moreover that the paths $p_{x}, p_{y}$ merge at a vertex $m$ before hitting $\gamma_{1}$ or exactly when hitting $\gamma_{1}$. Then, the edge configuration consisting of the edge $e$ and the part of $p_{x}$ and $p_{y}$ from $x$ (resp. $y$ ) to $m$ consists of:

Case 1a: either a trivial cycle,

Case 1b: or a non-trivial cycle parallel to $\gamma_{1}$.
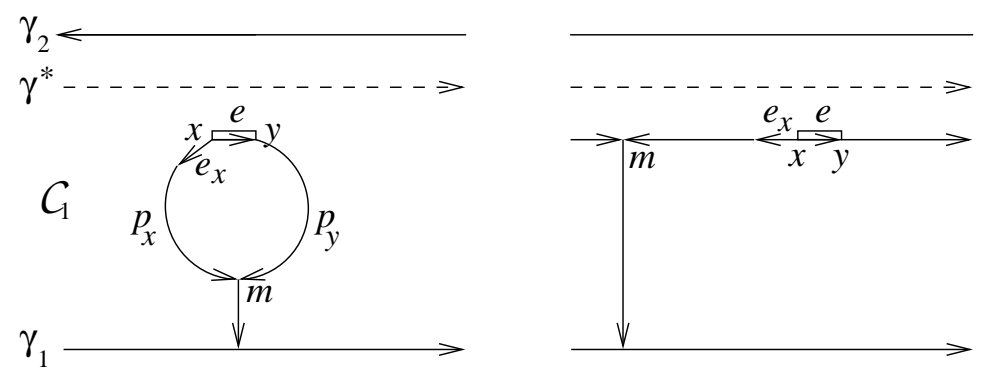

Figure 11: The edge configuration $F \cup\{e\}$ in Case $1 a$ (left) and Case $1 b$ (right).

Case 2: As in Case 1, the edge $e$ belongs to the cylinder $\mathscr{C}_{1}$, but this time the paths $p_{x}, p_{y}$ do not merge before hitting $\gamma_{1}$, see Figure 12. Then cutting along $\{e\} \cup\left\{p_{x}\right\} \cup\left\{p_{y}\right\}$ separates the cylinder $\mathscr{C}_{1}$ into two connected components, one homeomorphic to a disc and the other to a cylinder. We let $m$ be the unique boundary vertex of the component homeomorphic to a disc, with two incoming edges. 


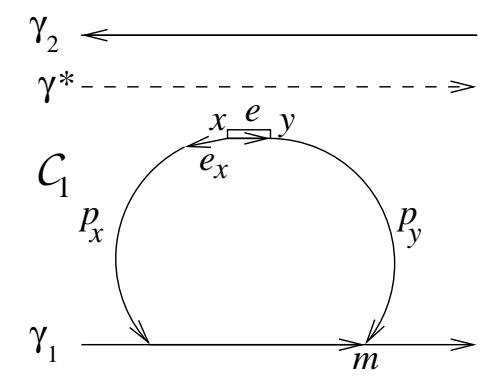

Figure 12: The edge configuration $F \cup\{e\}$ in Case 2 .

Case 3: The edge $e$ crosses the non-trivial cycle $\gamma^{*}$, see Figure 13. Then, up to relabeling, the vertex $x$ (resp. $y$ ) is connected to the path $\gamma_{1}$ (resp. $\gamma_{2}$ ), and the path $p_{x}$ (resp. $p_{y}$ ) is contained in the cylinder $\mathscr{C}_{1}\left(\right.$ resp. $\left.\mathscr{C}_{2}\right)$. We let $m$ be the vertex at which the path $\gamma_{x}$ hits the non-trivial cycle $\gamma_{1}$.

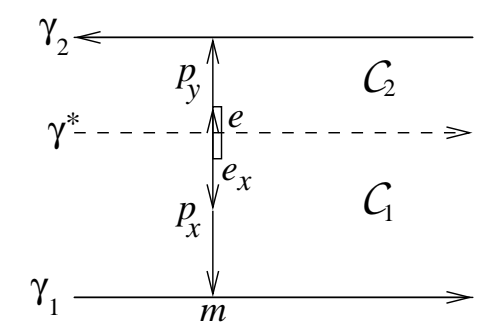

Figure 13: The edge configuration $F \cup\{e\}$ in Case 3 .

As a consequence, we obtain the following characterization of the edge configuration $F_{\left\{e, e_{x}\right\}}$.

Lemma 20. Let $F$ be an OCRSF of $G_{1}, e=(x, y)$ be an edge of $E\left(G_{1}\right) \backslash F$, and $e_{x}$ be the unique edge of $F$ exiting the vertex $x$. Then using the above case splitting, we have:

1. In all cases, as long as $x \neq m$, then the edge configuration $F_{\left\{e, e_{x}\right\}}$ is an OCRSF whose oriented non-trivial cycles are in bijection with those of $F$, so that $F$ and $F_{\left\{e, e_{x}\right\}}$ have the same reference number and $N\left(F_{\left\{e, e_{x}\right\}}\right)=N(F)$.

2. When $x=m$, i.e. the path $p_{x}$ is reduced to the point $m$, refer to Figure 14:

- In Cases $1 a$ and 2 , a trivial cycle is created so that $F_{\left\{e, e_{x}\right\}}$ is not an OCRSF.

- In Case 1b: when $x=m \notin \gamma_{1}$, then a non-trivial cycle parallel to $\gamma_{1}$ is created, so that $F_{\left\{e, e_{x}\right\}}$ is an OCRSF with the same reference number as $F$, 
satisfying $N\left(F_{\left\{e, e_{x}\right\}}\right)=N(F) \pm 1$. When $x=m \in \gamma_{1}$, then a non-trivial cycle parallel to $\gamma_{1}$ is created and $\gamma_{1}$ is broken up. Thus, $F_{\left\{e, e_{x}\right\}}$ is an OCRSF with the same reference number as $F$, satisfying $N\left(F_{\left\{e, e_{x}\right\}}\right)=N(F) \pm 2$ or $N\left(F_{\left\{e, e_{x}\right\}}\right)=N(F)$.

- In Case 3: as long as the number $n$ of non-trivial cycles is $\geq 2$, then the non-trivial cycle $\gamma_{1}$ is broken up, so that $F_{\left\{e, e_{x}\right\}}$ is an OCRSF with the same reference number as $F$, satisfying $N\left(F_{\left\{e, e_{x}\right\}}\right)=N(F) \pm 1$. When $n=1$, then the unique non-trivial cycle is broken up, and a non-trivial cycle orthogonal to $\gamma_{1}$ is created. Thus $F_{\left\{e, e_{x}\right\}}$ has a different reference number than $F$.

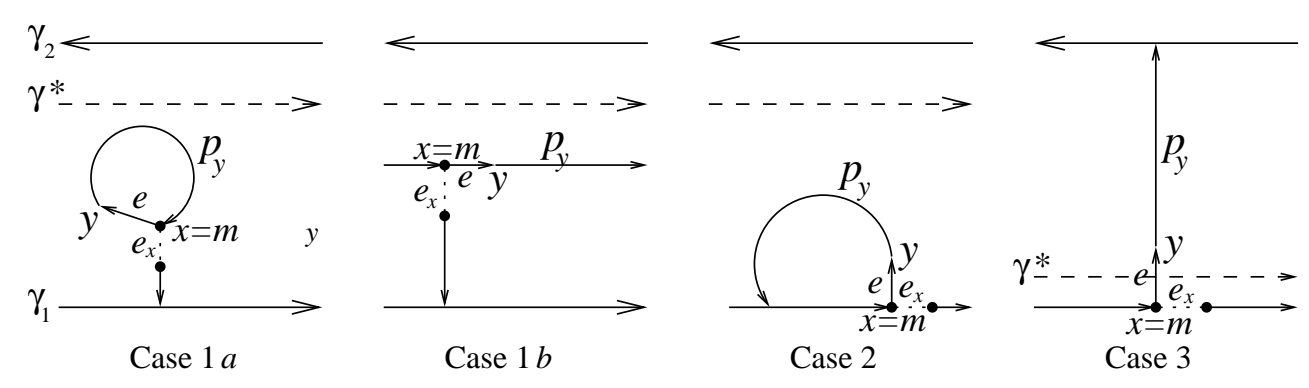

Figure 14: The edge configuration $F_{\left\{e, e_{x}\right\}}$, when $x=m$.

\subsection{Licit primal/dual edge moves}

Let $\left(F, F^{*}\right)$ be a pair of dual OCRSFs of $G_{1}$ and $G_{1}^{*}$. We consider an edge $e_{1}=\left(x_{1}, y_{1}\right)$ of $E\left(G_{1}\right) \backslash F$, and an edge $e_{2}^{*}=\left(x_{2}^{*}, y_{2}^{*}\right)$ of $E\left(G_{1}^{*}\right) \backslash F^{*}$. Then, $e_{1}$ is the dual of an oriented edge $e_{1}^{*}=\left(x_{1}^{*}, y_{1}^{*}\right)$ of $F^{*}$, and $e_{2}^{*}$ is the dual of an oriented edge $e_{2}=\left(x_{2}, y_{2}\right)$ of $F$.

Definition 5.1. We say that the pair of primal and dual oriented edge configurations $\left(F_{\left\{e_{1}, e_{2}\right\}}, F_{\left\{e_{2}^{*}, e_{1}^{*}\right\}}^{*}\right)$ is obtained from $\left(F, F^{*}\right)$ by a primal/dual edge move. This move is called licit, whenever:

$$
x_{1}=x_{2} \quad \text { and } \quad x_{1}^{*}=x_{2}^{*} .
$$

Proposition 21. The pair $\left(F_{\left\{e_{1}, e_{2}\right\}}, F_{\left\{e_{2}^{*}, e_{1}^{*}\right\}}\right)$ consists of dual OCRSFs of $G_{1}$ and $G_{1}^{*}$, iff the primal/dual edge move is licit. When this is the case,

1. either the pair $\left(F_{\left\{e_{1}, e_{2}\right\}}, F_{\left\{e_{2}^{*}, e_{1}^{*}\right\}}^{*}\right)$ has the same reference number as $\left(F, F^{*}\right)$, and

$$
N\left(F_{\left\{e_{1}, e_{2}\right\}}, F_{\left\{e_{2}^{*}, e_{1}^{*}\right\}}^{*}\right)=N\left(F, F^{*}\right),
$$

2. or the pairs $\left(F_{\left\{e_{1}, e_{2}\right\}}, F_{\left\{e_{2}^{*}, e_{1}^{*}\right\}}^{*}\right)$ and $\left(F, F^{*}\right)$ have different reference numbers, and

$$
N\left(F_{\left\{e_{1}, e_{2}\right\}}, F_{\left\{e_{2}^{*}, e_{1}^{*}\right\}}^{*}\right)=N\left(F, F^{*}\right)=0 .
$$


Proof. Let us first show that Condition (9) is necessary. By construction of $F_{\left\{e_{1}, e_{2}\right\}}$, the outgoing edge $e_{1}$ is added at the vertex $x_{1}$. Since $F$ is an OCRSF, the vertex $x_{1}$ has a unique outgoing edge $e_{x_{1}}$ of $F$. Suppose that $x_{1} \neq x_{2}$, this implies that $e_{2} \neq e_{x_{1}}$ so that the vertex $x_{1}$ has two outgoing edges in the edge configuration $F_{\left\{e_{1}, e_{2}\right\}}$. By Remark 2, this forbids $F_{\left\{e_{1}, e_{2}\right\}}$ from being an OCRSF. A similar argument holds for $F_{\left\{e_{2}^{*}, e_{1}^{*}\right\}}^{*}$, when $x_{1}^{*} \neq x_{2}^{*}$.

Let us now suppose that $x_{1}=x_{2}$ and $x_{1}^{*}=x_{2}^{*}$, see Figure 15 .

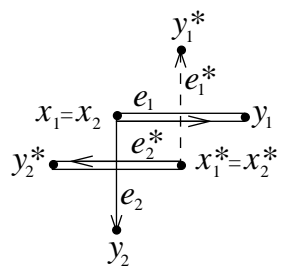

Figure 15: The edges $e_{1}, e_{2}, e_{1}^{*}, e_{2}^{*}$, when $x_{1}=x_{2}, x_{1}^{*}=x_{2}^{*}$.

In the sequel, we use the notations of Section 5.2. Suppose that $x_{1} \neq m_{1}$, and $x_{2}^{*} \neq m_{2}^{*}$. Then, by Point 1. of Lemma 20, the edge configuration $F_{\left\{e_{1}, e_{2}\right\}}\left(\right.$ resp. $\left.F_{\left\{e_{2}^{*}, e_{1}^{*}\right\}}\right)$ has the same reference number as $F\left(\right.$ resp. $\left.F^{*}\right)$ and $N\left(F_{\left\{e_{1}, e_{2}\right\}}\right)=N(F)\left(\operatorname{resp} . N\left(F_{\left\{e_{2}^{*}, e_{1}^{*}\right\}}^{*}\right)=\right.$ $N\left(F^{*}\right)$ ), so that Point 1 . of Proposition 21 is clearly satisfied.

Suppose now that $x_{1}=m_{1}$, then in Cases $1 a$ and 2 , the edge-path $p_{y_{1}}$ hits the vertex $x_{1}$, and $\left\{e_{1}\right\} \cup\left\{p_{y_{1}}\right\}$ contains a trivial cycle, see Figure 16. In the dual graph, the edgepath $p_{x_{1}^{*}}$ must enter this trivial cycle, which is impossible since $p_{x_{1}^{*}}$ must also connect $x_{1}^{*}$ to one of the dual non-trivial cycles, so that this case cannot occur.

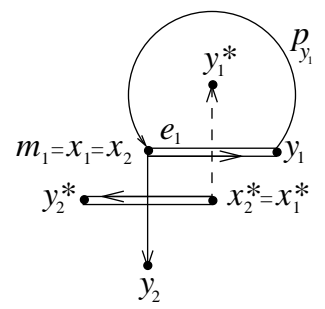

Figure 16: In Cases $1 a$ and 2, when $x_{1}=m_{1}$, the edge-path $p_{y_{1}}$ hits $x_{1}$, and $\left\{e_{1}\right\} \cup\left\{p_{y_{1}}\right\}$ contains a trivial cycle.

In Case $1 b$, the edge-path $p_{y_{1}}$ hits the vertex $x_{1}$, and $\left\{e_{1}\right\} \cup\left\{p_{y_{1}}\right\}$ contains a nontrivial cycle parallel to $\gamma_{1}$, see Figure 17 (left). Suppose now that $x_{1}=m_{1} \notin \gamma_{1}$, then in the dual graph, the edge path $p_{y_{2}^{*}}$ must hit $x_{2}^{*}$ (implying that $x_{2}^{*}=m_{2}^{*}$ ), and $\left\{e_{2}^{*}\right\} \cup\left\{p_{y_{2}^{*}}\right\}$ must contain a non-trivial cycle parallel to $\gamma_{1}$, implying that the nontrivial cycles contained in $\left\{e_{1}\right\} \cup\left\{p_{y_{1}}\right\}$ and $\left\{e_{2}^{*}\right\} \cup\left\{p_{y_{2}^{*}}\right\}$ are parallel and in opposite 
directions, see Figure 17 (right). Thus, by Point 2., Case $1 b$ of Lemma 20, we know that $\left(F_{\left\{e_{1}, e_{2}\right\}}, F_{\left\{e_{2}^{*}, e_{1}^{*}\right\}}^{*}\right)$ are dual OCRSFs of $G_{1}$ and $G_{1}^{*}$, with the same reference number as $\left(F, F^{*}\right)$, such that:

$$
N\left(F_{\left\{e_{1}, e_{2}\right\}}, F_{\left\{e_{2}^{*}, e_{1}^{*}\right\}}^{*}\right)=N(F) \pm 1+N\left(F^{*}\right) \mp 1=N\left(F, F^{*}\right) .
$$

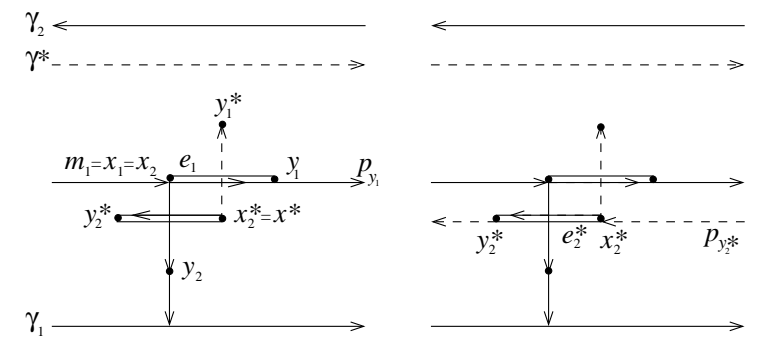

Figure 17: Left: In Cases 1b, when $x_{1}=m_{1}$, the edge-path $p_{y_{1}}$ hits $x_{1}$, and $\left\{e_{1}\right\} \cup\left\{p_{y_{1}}\right\}$ contains a non-trivial cycle. Right: when $x_{1}=m_{1} \notin \gamma_{1}$, the edge path $p_{y_{2}^{*}}$ must hit $x_{2}^{*}$, and $\left\{e_{2}^{*}\right\} \cup\left\{p_{y_{2}^{*}}\right\}$ contains a non-trivial cycle, with opposite direction.

In Case $1 b$, when $x_{1}=m_{1} \in \gamma_{1}$, then $\left\{e_{1}\right\} \cup\left\{p_{y_{1}}\right\}$ and $\gamma_{1}$ must be co-oriented, so that by Point 2., Case $1 b$ of Lemma 20, we know that $F_{\left\{e_{1}, e_{2}\right\}}$ is an OCRSF, in which the non-trivial cycle $\gamma_{1}$ is broken up and the non-trivial cycle $\left\{e_{1}\right\} \cup\left\{p_{y_{1}}\right\}$ is added, implying that $F_{\left\{e_{1}, e_{2}\right\}}$ has the same reference number as $F$, and $N\left(F_{\left\{e_{1}, e_{2}\right\}}\right)=N(F)$. In the dual graph, we have $x_{2}^{*} \neq m_{2}^{*}$, so that by Point 1 . of Lemma 20 , we know that $F_{\left\{e_{2}^{*}, e_{1}^{*}\right\}}$ is an OCRSF whose non-trivial cycles are in bijection with those of $F$. Summing the two contributions $N\left(F_{\left\{e_{1}, e_{2}\right\}}\right)$ and $N\left(F_{\left\{e_{2}^{*}, e_{1}^{*}\right\}}\right)$, we deduce that Point 1. of Proposition 21 is satisfied.

In Case 3, when $x_{1}=m_{1}$, the edge $e_{1}^{*}$ must belong to $\gamma^{*}$ (implying that $x_{1}^{*}=x_{2}^{*}=m_{2}^{*}$ ), and the paths $\gamma^{*}$ and $\gamma_{1}$ must be in opposite directions, see Figure 18. If the number of non-trivial cycles of $F$ is $\geq 2$, then by Point 2., Case 3 of Lemma 20, we know that $F_{\left\{e_{1}, e_{2}\right\}}$ (resp. $F_{\left\{e_{2}^{*}, e_{1}^{*}\right\}}$ ) is an OCRSF, in which the non-trivial cycle $\gamma_{1}$ (resp. $\gamma^{*}$ ) is broken up. As a consequence, $\left(F_{\left\{e_{1}, e_{2}\right\}}, F_{\left\{e_{2}^{*}, e_{1}^{*}\right\}}\right)$ are dual OCRSFs of $G_{1}$ and $G_{1}^{*}$ with the same reference number as $\left(F, F^{*}\right)$ such that:

$$
N\left(F_{\left\{e_{1}, e_{2}\right\}}, F_{\left\{e_{2}^{*}, e_{1}^{*}\right\}}^{*}\right)=N(F) \pm 1+N\left(F^{*}\right) \mp 1=N\left(F, F^{*}\right) .
$$

When the number of non-trivial cycles of $F$ is 1 , since $\gamma^{*}$ and $\gamma_{1}$ must be in opposite directions, we know that $N\left(F, F^{*}\right)=0$. Moreover, by Point 2., Case 3 of Lemma 20, we know that $F_{\left\{e_{1}, e_{2}\right\}}$ (resp. $F_{\left\{e_{2}^{*}, e_{1}^{*}\right\}}$ ) is an OCRSF, in which the nontrivial cycle $\gamma_{1}$ (resp. $\left.\gamma^{*}\right)$ is broken up and in which a non-trivial cycle orthogonal to the original one is created. Since these must have opposite directions, we deduce that the pair $\left(F_{\left\{e_{1}, e_{2}\right\}}, F_{\left\{e_{2}^{*}, e_{1}^{*}\right\}}^{*}\right)$ has a different reference number than $\left(F, F^{*}\right)$, and 


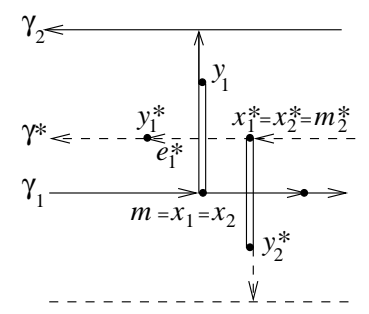

Figure 18: In Case 3, when $x_{1}=m_{1}$, the edge $e_{1}^{*}$ must belong to $\gamma^{*}$, and the paths $\gamma^{*}$ and $\gamma_{1}$ must be in opposite directions

$N\left(F_{\left\{e_{1}, e_{2}\right\}}, F_{\left\{e_{2}^{*}, e_{1}^{*}\right\}}^{*}\right)=0=N\left(F, F^{*}\right)$, implying that Point 2. of Proposition 21 is satisfied.

\section{Proof of Theorem 17}

Let $G$ be an infinite, $\mathbb{Z}^{2}$-periodic isoradial graph, $\mathcal{G}$ be the corresponding Fisher graph, and $G_{1}, \mathcal{G}_{1}$ be their respective fundamental domains. As a consequence of Lemma 19 and using notations introduced in Section 4.1, we deduce that the critical Laplacian characteristic polynomial $P_{\text {Lap }}(\mathrm{z}, \mathrm{w})$, and the critical dimer characteristic polynomial $P_{\text {dimer }}^{0}(\mathrm{z}, \mathrm{w})$, can be rewritten as:

$$
\begin{aligned}
P_{\text {Lap }}(\mathrm{z}, \mathrm{w}) & =\sum_{\left(\mathbf{F}, \mathbf{F}^{*}\right) \in \mathcal{F}\left(G_{1}, G_{1}^{*}\right)}\left(\prod_{\mathbf{e}=\mathbf{x} \mathbf{y} \in \mathbf{F}} \tan \theta_{\mathbf{x y}}\right)\left(-\mathbf{z}^{h_{0}} \mathbf{w}^{v_{0}}\right)^{\frac{1}{2}} N\left(\mathbf{F}, \mathbf{F}^{*}\right) \\
P_{\text {dimer }}^{0}(\mathbf{z}, \mathbf{w}) & =\sum_{\left(F, F^{*}\right) \in \mathcal{F}^{0}\left(\mathcal{G}_{1}, \mathcal{G}_{1}^{*}\right)}\left(\prod_{e=(x, y) \in F} f_{x} \overline{f_{y}} K_{x, y}\right)\left(-\mathbf{z}^{h_{0}} \mathbf{w}^{v_{0}}\right)^{\frac{1}{2} N\left(F, F^{*}\right)} .
\end{aligned}
$$

In Section 6.1, we prove the first part of Theorem 17 by constructing pairs of essential OCRSFs of $\mathcal{G}_{1}$ and $\mathcal{G}_{1}^{*}$ counted by $P_{\text {dimer }}^{0}(\mathrm{z}, \mathrm{w})$ from pairs of dual OCRSFs of $G_{1}$ and $G_{1}^{*}$ counted by $P_{\text {Lap }}(\mathrm{z}, \mathrm{w})$, see Theorem 29. Then, in Section 6.2 , we prove that this construction is weight preserving, thus ending the proof of Theorem 17 .

\subsection{Explicit construction}

Let us start by giving the general idea of the construction. Let $\left(\mathbf{F}, \mathbf{F}^{*}\right)$ be a pair of dual OCRSFs of $G_{1}$ and $G_{1}^{*}$, then to $\left(\mathbf{F}, \mathbf{F}^{*}\right)$ we assign a family $\mathcal{S}\left(\mathbf{F}, \mathbf{F}^{*}\right)$ consisting of pairs of dual essential OCRSFs of $\mathcal{G}_{1}$ and $\mathcal{G}_{1}^{*}$, such that:

1. $\bigcup_{\left(\mathbf{F}, \mathbf{F}^{*}\right) \in \mathcal{F}\left(G_{1}, G_{1}^{*}\right)} \mathcal{S}\left(\mathbf{F}, \mathbf{F}^{*}\right)=\mathcal{F}^{0}\left(\mathcal{G}_{1}, \mathcal{G}_{1}^{*}\right)$. 
2. When $\left(\mathbf{F}_{1}, \mathbf{F}_{1}^{*}\right) \neq\left(\mathbf{F}_{2}, \mathbf{F}_{2}^{*}\right)$, then $\mathcal{S}\left(\mathbf{F}_{1}, \mathbf{F}_{1}^{*}\right) \cap \mathcal{S}\left(\mathbf{F}_{2}, \mathbf{F}_{2}^{*}\right)=\emptyset$.

3. For every $\left(F, F^{*}\right) \in \mathcal{S}\left(\mathbf{F}, \mathbf{F}^{*}\right)$, then

- either the reference number of $\left(F, F^{*}\right)$ in $\mathcal{G}_{1}, \mathcal{G}_{1}^{*}$ is equal to the reference number of $\left(\mathbf{F}, \mathbf{F}^{*}\right)$ in $G_{1}, G_{1}^{*}$, and $N_{\mathcal{G}_{1}, \mathcal{G}_{1}^{*}}\left(F, F^{*}\right)=N_{G_{1}, G_{1}^{*}}\left(\mathbf{F}, \mathbf{F}^{*}\right)$,

- or the pairs $\left(F, F^{*}\right)$ and $\left(\mathbf{F}, \mathbf{F}^{*}\right)$ have different reference numbers, and $N_{\mathcal{G}_{1}, \mathcal{G}_{1}^{*}}\left(F, F^{*}\right)=N_{G_{1}, G_{1}^{*}}\left(\mathbf{F}, \mathbf{F}^{*}\right)=0$,

where we have added a subscript to the signed number of cycles $N(\cdot)$ to indicate on which graph it is computed.

For every pair $\left(\mathbf{F}, \mathbf{F}^{*}\right)$ of dual OCRSFs of $G_{1}$ and $G_{1}^{*}$, loosely stated, the family $\mathcal{S}\left(\mathbf{F}, \mathbf{F}^{*}\right)$ is constructed as follows. Let $\mathbf{e}_{1}, \cdots, \mathbf{e}_{m}$, be an arbitrary labeling of unoriented edges of $E\left(G_{1}\right) \backslash \mathbf{F}$. For $k \in\{0, \cdots, m\}$, we let $J_{k}=\left\{\left(i_{1}, \cdots, i_{k}\right) \in\{1, \cdots, m\}^{k} \mid 1 \leq i_{1}<\right.$ $\left.\cdots<i_{k} \leq m\right\}$, with the convention that $J_{k}=\emptyset$, when $k=0$. Then,

$$
\mathcal{S}\left(\mathbf{F}, \mathbf{F}^{*}\right)=\bigcup_{k=0}^{m} \bigcup_{\left(i_{1}, \cdots, i_{k}\right) \in J_{k}} \mathcal{F}^{\left(\mathbf{F}, \mathbf{F}^{*}\right),\left\{\mathbf{e}_{i_{1}}, \cdots, \mathbf{e}_{i_{k}}\right\}}\left(\mathcal{G}_{1}, \mathcal{G}_{1}^{*}\right),
$$

where $\mathcal{F}^{\left(\mathbf{F}, \mathbf{F}^{*}\right),\left\{\mathbf{e}_{i_{1}}, \cdots, \mathbf{e}_{i_{k}}\right\}}\left(\mathcal{G}_{1}, \mathcal{G}_{1}^{*}\right)$ is constructed by induction on $k$ using licit primal/dual moves, introduced in Section 5. In Section 6.1.1, we prove all results needed for the initial step of the induction. Then, in Section 6.1.2, we specify licit primal/dual moves to pairs of essential OCRSFs of $\mathcal{G}_{1}$ and $\mathcal{G}_{1}^{*}$. This allows us to precisely define the set $\mathcal{F}^{\left(\mathbf{F}, \mathbf{F}^{*}\right),\left\{\mathbf{e}_{i_{1}}, \cdots, \mathbf{e}_{i_{k}}\right\}}\left(\mathcal{G}_{1}, \mathcal{G}_{1}^{*}\right)$ in Section 6.1.3. Finally, we state and prove Theorem 29 establishing that we exactly obtain all pairs of dual essential CRSFs of $\mathcal{G}_{1}$ and $\mathcal{G}_{1}^{*}$.

\subsubsection{Initial step of the induction}

Let $\mathbf{F}$ be a subset of oriented edges of $G_{1}$ such that each vertex has exactly one outgoing edge of $\mathbf{F}$. Considering $\mathbf{F}$ as a subset of long edges of $\mathcal{G}_{1}$ defines, for every decoration $\mathbf{x}$, a unique root vertex $v_{1}(\mathbf{x})$. From now on, whenever no confusion occurs, we omit the argument $\mathbf{x}$. For the whole of this section, we let $\tau \in\{c w, c c l w\}^{V\left(G_{1}\right)}$ be an assignment of type $c w$ or $c c l w$ to vertices of $G_{1}$.

Lemma 22. A subset of oriented edges $F$ of $\mathcal{G}_{1}$ is an essential OCRSF compatible with $\mathbf{F}$ and $\tau$, iff:

1. $\mathbf{F}$ is an $O C R S F$ of $G_{1}$,

2. long edges of $F$ are exactly those of $\mathbf{F}$,

3. the restriction of $F$ to every decoration $\mathbf{x}$ contains:

- all inner edges with the orientation induced by the type $\tau(\mathbf{x})$, 
- the edge $\left(w_{1}, v_{1}\right)$ at the triangle $t_{1}$ if the decoration is of type $\mathrm{cw}$, or the edge $\left(z_{1}, v_{1}\right)$ if it is of type cclw,

- for every $i \neq 1$, any of the three possible 2-edge configuration of Definition 4.1, at the triangle $t_{i}$, with the orientation induced by the type, see also Figure 9.

Moreover, when this is the case, oriented non-trivial cycles of $F$ in $\mathcal{G}_{1}$ are in bijection with oriented non-trivial cycles of $\mathbf{F}$ in $G_{1}$.

Proof. Since each vertex of $G_{1}$ has a unique outgoing edge of $\mathbf{F}$, we know that $\mathbf{F}$ is an OCRSF of $G_{1}$, iff it contains no trivial cycle. Moreover, we know that $F$ is an essential OCRSF of $\mathcal{G}_{1}$ compatible with $\mathbf{F}$ and $\tau$, iff it satisfies Conditions 1.2.3. of Corollary 15. Since every decoration of $\mathcal{G}_{1}$ has a unique root vertex, Condition 3. can be rewritten as Condition 3. of Lemma 22 above. Thus, it remains to show that $F$ contains no trivial cycle consisting of long and short edges of $\mathcal{G}_{1}$, iff $\mathbf{F}$ contains no trivial cycle of $G_{1}$, or equivalently $F$ contains a trivial cycle consisting of long and short edges iff $\mathbf{F}$ contains a trivial cycle. Because of the geometry of $\mathcal{G}_{1}$, the direction from left to right is straightforward. Conversely, suppose that $\mathbf{F}$ contains a trivial cycle. Then, since every vertex of $G_{1}$ has a unique outgoing edge of $\mathbf{F}$ this implies that the trivial cycle must be co-oriented. Now, consider an oriented configuration of $\mathcal{G}_{1}$ satisfying Conditions 2. and 3. above. Since every decoration of $\mathcal{G}_{1}$ has a unique root vertex, this means that every vertex of the decoration $\mathbf{x}$ is connected by a unique path to the root vertex $v_{1}(\mathbf{x})$, in particular this holds for every vertex of type ' $v$ ' of the decoration. Thus, if $\mathbf{F}$ contains a trivial cycles, then $F$ contains a trivial cycle consisting of long and short edges. A similar argument shows that if $F$ is an OCRSF of $\mathcal{G}_{1}$, its non-trivial cycles are in bijection with those of $\mathbf{F}$ in $G_{1}$.

Figure 19 below illustrates in an example all essential OCRSFs of $\mathcal{G}_{1}$ compatible with $\mathbf{F}$ and $\tau$.
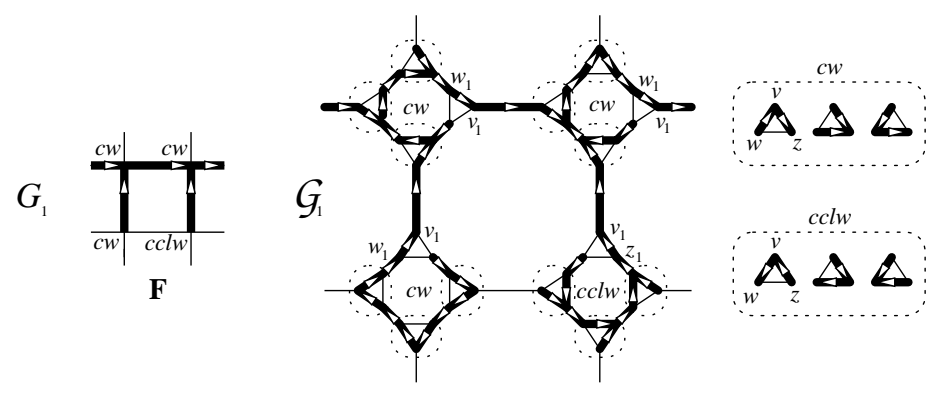

Figure 19: Left: an OCRSF $\mathbf{F}$ of $G_{1}$ and an assignment of types to vertices of $G_{1}$. Right: essential OCRSFs of $\mathcal{G}_{1}$ compatible with $\mathbf{F}$ and $\tau$. 
We now study properties of dual OCRSFs. Observe that the dual graph $G_{1}^{*}$ is a subgraph of $\mathcal{G}_{1}^{*}$, see also Figure 20 below. Since inner edges of the decorations are always present in essential OCRSFs of $\mathcal{G}_{1}$, we omit their dual edges in our picture of $\mathcal{G}_{1}^{*}$.
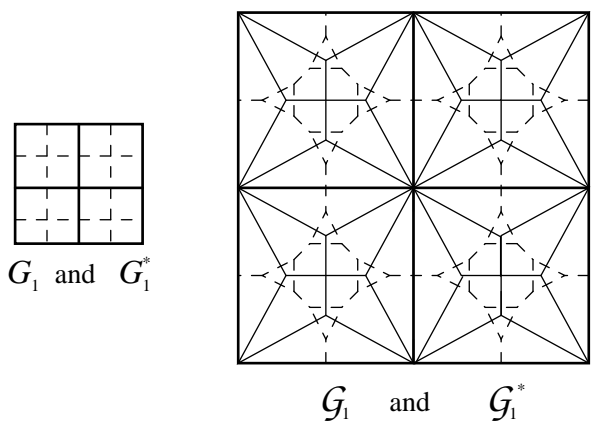

Figure 20: Left: the graph $G_{1}$ (dotted lines) and it dual $G_{1}^{*}$ (full lines). Right: the graph $\mathcal{G}_{1}$ (dotted lines) and its dual $\mathcal{G}_{1}^{*}$ (full lines), without dual edges of inner edges of the decorations.

Let $\mathbf{F}$ be an OCRSF of $G_{1}$ and $F$ be an essential OCRSF of $\mathcal{G}_{1}$ compatible with $\mathbf{F}$ and $\tau$. Suppose for the moment that edges are unoriented, and denote by $F^{*}$ the dual CRSF of $F$ in $\mathcal{G}_{1}^{*}$, and by $\mathbf{F}^{*}$ the dual CRSF of $\mathbf{F}$ in $G_{1}^{*}$. Then, the restriction of $F^{*}$ to dual long edges is $\mathbf{F}^{*}$, and since $G_{1}^{*}$ is a subgraph of $\mathcal{G}_{1}^{*}$, the CRSF $\mathbf{F}^{*}$ is a subgraph of $F^{*}$.

Moreover, by Lemma 22, we know that non-trivial cycles of $F$ in $\mathcal{G}_{1}$ and $\mathbf{F}$ in $G_{1}$ are in bijection, implying that non-trivial cycles of $F^{*}$ in $\mathcal{G}_{1}^{*}$ and $\mathbf{F}^{*}$ in $G_{1}^{*}$ are also in bijection. As a consequence, the non-trivial cycles of $F^{*}$ in $\mathcal{G}_{1}^{*}$ are exactly the non trivial cycles of $\mathbf{F}^{*}$ in $G_{1}^{*}$, and branches of $\mathbf{F}^{*}$ in $G_{1}^{*}$ are also branches of $F^{*}$ in $\mathcal{G}_{1}^{*}$. Recalling that OCRSFs are obtained from a CRSF by orienting branches towards the non-trivial cycles, and orienting each of the non-trivial cycles in one of the two possible ways, we have shown the following lemma describing oriented versions of $F^{*}$ and $\mathbf{F}^{*}$.

Lemma 23. Let $\mathbf{F}$ be an OCRSF of $G_{1}$ and $F$ be an essential OCRSF of $\mathcal{G}_{1}$ compatible with $\mathbf{F}$ and $\tau$. Then, if $F^{*}$ is a dual OCRSF of $F$, the restriction $\mathbf{F}^{*}$ of $F^{*}$ to dual long edges is an OCRSF of $G_{1}^{*}$, and oriented non-trivial cycles of $F^{*}$ in $\mathcal{G}_{1}^{*}$ are exactly those of $\mathbf{F}^{*}$ in $G_{1}^{*}$. Conversely, let $\mathbf{F}^{*}$ be a dual OCRSF of $\mathbf{F}$, then there is a unique dual OCRSF $F^{*}$ of $F$ in $\mathcal{G}_{1}^{*}$ such that the restriction of $F^{*}$ to dual long edges is $\mathbf{F}^{*}$.

Figure 21 below illustrates a dual OCRSF $\mathbf{F}^{*}$ of Figure 19, and the unique dual OCRSF of $F$ of Figure 19, whose restriction to dual long edges is $\mathbf{F}^{*}$. 


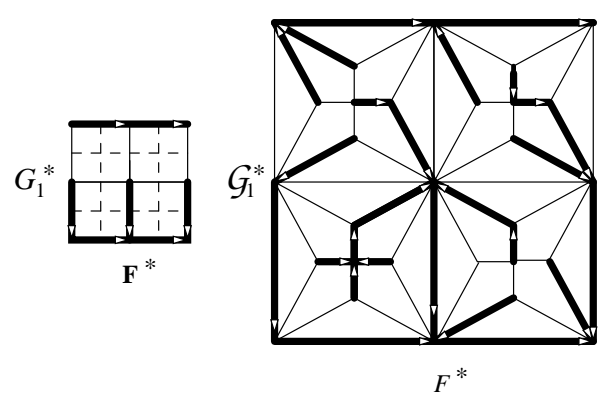

Figure 21: Left: A dual OCRSF $\mathbf{F}^{*}$ of $\mathbf{F}$ of Figure 19. Right: the unique dual OCRSF $F^{*}$ of $F$ of Figure 19 whose restriction to dual long edges is $\mathbf{F}^{*}$.

Definition 6.1. Let $\left(\mathbf{F}, \mathbf{F}^{*}\right)$ be a pair of dual OCRSFs of $G_{1}$ and $G_{1}^{*}$. Then, define $\mathcal{F}^{\tau,\left(\mathbf{F}, \mathbf{F}^{*}\right)}\left(\mathcal{G}_{1}, \mathcal{G}_{1}^{*}\right)$ to be the set of pairs $\left(F, F^{*}\right)$ of essential dual OCRSFs of $\mathcal{G}_{1}$ and $\mathcal{G}_{1}^{*}$, compatible with $\left(\mathbf{F}, \mathbf{F}^{*}\right)$ and $\tau$, that is the set of pairs $\left(F, F^{*}\right)$, such that $F$ is an essential OCRSF of $\mathcal{G}_{1}$ compatible with $\mathbf{F}$ and $\tau$, and $F^{*}$ is the unique dual OCRSF of $F$ whose restriction to dual long edges is $\mathbf{F}^{*}$.

As a consequence of Lemmas 22 and 23, we have the following.

Corollary 24. The set $\mathcal{F}^{\tau,\left(\mathbf{F}, \mathbf{F}^{*}\right)}\left(\mathcal{G}_{1}, \mathcal{G}_{1}^{*}\right)$ is well defined and non-empty. Moreover, the reference number in $\mathcal{G}_{1}$ of every pair $\left(F, F^{*}\right)$ in this set is equal to the reference number in $G_{1}$ of $\left(\mathbf{F}, \mathbf{F}^{*}\right)$ in $G_{1}$, and

$$
N_{\mathcal{G}_{1}, \mathcal{G}_{1}^{*}}\left(F, F^{*}\right)=N_{G_{1}, G_{1}^{*}}\left(\mathbf{F}, \mathbf{F}^{*}\right)
$$

\subsubsection{Essential moves and reverse moves}

Let $\mathbf{L}$ be a subset of oriented edges of $G_{1}$ also considered as a subset of long edges of $\mathcal{G}_{1}$, satisfying $(*)$ of Section 4.3.1 (that is every decoration of $\mathcal{G}_{1}$ has at least one root vertex of $\mathbf{L})$. For the whole of this section, we let $\tau \in\{c w, c c l w\}^{V\left(G_{1}\right)}$ be an assignment of types to vertices of $G_{1}$. Suppose that there exists an essential OCRSF $F$ of $\mathcal{G}_{1}$ compatible with $\mathbf{L}$ and $\tau$, and let $F^{*}$ be a dual OCRSF of $F$.

In this section, we characterize licit primal/dual moves performed on $\left(F, F^{*}\right)$, yielding a pair of essential dual OCRSFs of $\mathcal{G}_{1}$ and $\mathcal{G}_{1}^{*}$, such that the first component either has an additional long edge (essential move) or a long edge of $\mathbf{L}$ removed (essential reverse move).

Notation. For the remainder of the paper, we need to introduce a specific notation for oriented edges of $G_{1}$. Fix an arbitrary orientation of edges of $G_{1}$, and denote by e a generic unoriented edge, by $+\mathbf{e}$ the oriented edge compatible with the fixed orientation, and by $-\mathbf{e}$ the reverse oriented edge. 


\section{Essential moves}

Assume that $\mathbf{L} \neq E\left(G_{1}\right)$, and let $\mathbf{e}$ be an unoriented edge of $E\left(G_{1}\right) \backslash \mathbf{L}$. We now characterize licit primal/dual moves performed on $\left(F, F^{*}\right)$, which yield a pair of essential dual OCRSFs of $\mathcal{G}_{1}$ and $\mathcal{G}_{1}^{*}$, such that the first component is an essential OCRSF compatible with $\mathbf{L} \cup\{ \pm \mathbf{e}\}$ and $\tau$, i.e. belongs to $\mathcal{F}^{\tau, \mathbf{L} \cup\{ \pm \mathbf{e}\}}\left(\mathcal{G}_{1}\right)$.

Suppose first that we want to add the edge $+\mathbf{e}$ to $F$, corresponding to a long edge $\left(v_{k}(\mathbf{x}), v_{\ell}(\mathbf{y})\right)$ of $\mathcal{G}_{1}$, for some $k \in\left\{1, \cdots, d_{\mathbf{x}}\right\}, \ell \in\left\{1, \cdots, d_{\mathbf{y}}\right\}$, see Figure 22. Assume for the moment that $\mathbf{x}$ has type $\tau(\mathbf{x})=c w$, and let us omit the arguments $\mathbf{x}$ and $\mathbf{y}$, recalling that the index $k$ refers to $\mathbf{x}$, and the index $\ell$ to $\mathbf{y}$.

Since $\left(v_{k}, v_{\ell}\right)$ is absent in $F$, its dual edge $\left(x^{*}, y^{*}\right)$ is present in $F^{*}$. Let us first handle Case A, where $x^{*}$ is to the right of $\left(v_{k}, v_{\ell}\right)$, see Figure 22 (first two columns). Since $F$ is an essential OCRSF it has one of the three possible 2-edge configurations at the triangle of the vertex $v_{k}$, with the orientation induced by the type $\tau(\mathbf{x})=c w$. Since we want the resulting configuration to be an essential OCRSF compatible with $\tau$, this implies that after the move, the triangle $t_{k}$ must contain either the edge $\left(w_{k}, v_{k}\right)$ or the edge $\left(w_{k}, z_{k}\right)$. Then, see Figure 22 (first column), our only choice is to remove the edge $\left(v_{k}, z_{k}\right)$ in Cases AI and AII, and the edge $\left(v_{k}, w_{k}\right)$ in Case AIII. This constraint fully determines the dual edges which are removed/added, and we only allow the move when it is licit, i.e. when edges involved satisfy Condition (9), that is in Cases AI and AII, see Figure 22 (second column). In Case $B$, when $x^{*}$ is to the left of $\left(v_{k}, v_{\ell}\right)$, a similar argument holds and yields the last two columns of Figure 22.

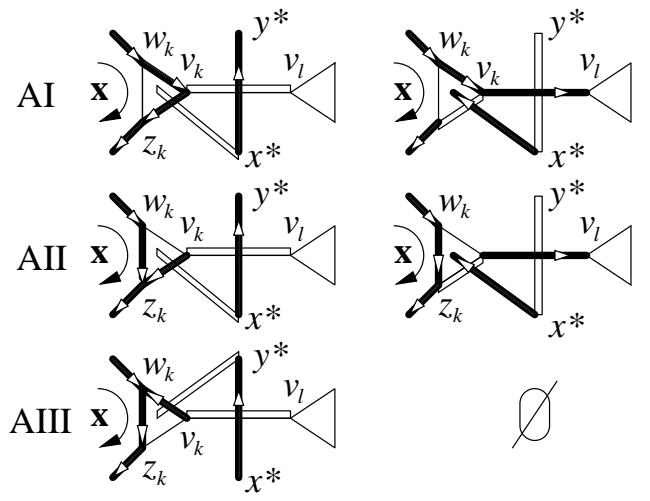

Case A

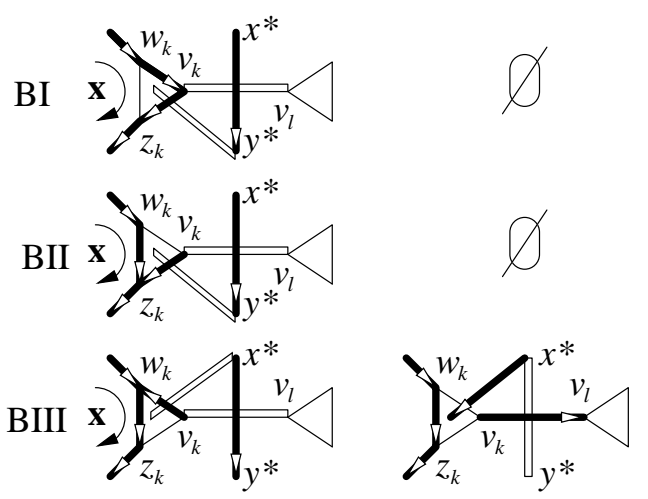

Case B

Figure 22: Adding the long edge $\left(v_{k}, v_{\ell}\right)$. First two columns: $x^{*}$ is to the right of $\left(v_{k}, v_{\ell}\right)$. Last two columns: $x^{*}$ is to the left of $\left(v_{k}, v_{\ell}\right)$.

By symmetry, when $\mathbf{x}$ has type $\tau(\mathbf{x})=c c l w$, if $x^{*}$ is to the right (resp. to the left) of $\left(v_{k}, v_{\ell}\right)$, we get Case B (resp. Case A) with ' $w$ 's and ' $z$ 's exchanged. Using symmetries again, the case where we add the edge $\left(v_{\ell}, v_{k}\right)$ to the OCRSF $F$ is also handled by 
Cases A and B. It is important to note that in each case at most one primal/dual move is allowed.

Definition 6.2. When the above move is allowed, it is called an essential move, and $\left(F, F^{*}\right)_{\{ \pm \mathbf{e}, \cdot\}}=\left(F_{\{ \pm \mathbf{e}, \cdot\}}, F_{\left\{\cdot, \pm \mathbf{e}^{*}\right\}}^{*}\right)$ denotes the resulting pair of OCRSFs. When it is not allowed, we set by convention, $\left(F, F^{*}\right)_{\{ \pm \mathbf{e}, \cdot\}}=\emptyset$.

Then, Proposition 21 and the construction of essential moves immediately yields the following.

Proposition 25. Let $F$ be an essential OCRSF of $\mathcal{G}_{1}$ compatible with $\mathbf{L}$ and $\tau$, and $F^{*}$ be a dual OCRSF of $F$. Then, the pair $\left(F, F^{*}\right)_{\{ \pm \mathbf{e}, \cdot\}}$ consists of:

- the empty set when the essential move is not allowed,

- when the essential move is allowed, a pair of dual essential OCRSFs of $\mathcal{G}_{1}$ and $\mathcal{G}_{1}^{*}$, such that $F$ is an essential OCRSF compatible with $\mathbf{L} \cup\{ \pm \mathbf{e}\}$ and $\tau$, and

1. either the pair $\left(F, F^{*}\right)_{\{ \pm \mathbf{e}, \cdot\}}$ has the same reference number as $\left(F, F^{*}\right)$, and

$$
N\left(\left(F, F^{*}\right)_{\{ \pm \mathbf{e}, \cdot\}}\right)=N\left(F, F^{*}\right),
$$

2. or the pairs $\left(F, F^{*}\right)_{\{ \pm \mathbf{e}, \cdot\}}$ and $\left(F, F^{*}\right)$ have different reference numbers, and

$$
N\left(\left(F, F^{*}\right)_{\{ \pm \mathbf{e}, \cdot\}}\right)=N\left(F, F^{*}\right)=0 .
$$

Moreover, the orientation of edges not involved in the move remains unchanged.

\section{Essential reverse moves}

Assume now that $\mathbf{L} \neq \emptyset$, and let $\epsilon \mathbf{e}=(\mathbf{x}, \mathbf{y})$ be an oriented edge of $\mathbf{L}$, where $\epsilon \in\{-,+\}$. We now characterize licit primal/dual moves performed on $\left(F, F^{*}\right)$, which yield a pair of essential OCRSFs of $\mathcal{G}_{1}$ and $\mathcal{G}_{1}^{*}$, such that the first component is an essential OCRSF compatible with $\mathbf{L} \backslash\{\epsilon \mathbf{e}\}$ and $\tau$, i.e. belongs to $\mathcal{F}^{\tau, \mathbf{L} \backslash\{\epsilon \mathbf{e}\}}\left(\mathcal{G}_{1}\right)$.

The edge $\epsilon \mathbf{e}$ corresponds to a long edge $\left(v_{k}(\mathbf{x}), v_{\ell}(\mathbf{y})\right)$ of $\mathcal{G}_{1}$, see Figure 23. Assume for the moment that $\mathbf{x}$ is of type $\tau(\mathbf{x})=c w$, and let us omit the arguments $\mathbf{x}$ and $\mathbf{y}$ onwards. Since $F$ is an essential OCRSF, it contains either the edge $\left(w_{k}, v_{k}\right)$ or the edge $\left(w_{k}, z_{k}\right)$ at the triangle $t_{k}$. Note that the edge $z_{k} v_{k}$ is always absent, so that its dual edge is present in $F^{*}$. Let us first handle Case $\mathrm{C}$, where this dual edge is oriented towards the triangle $t_{k}$.

Since we want the resulting configuration to be an essential OCRSF compatible with $\tau$, this implies that after the move, the triangle $t_{k}$ must contain one of the three possible 2-edge configurations, with the orientation induced by $\tau(\mathbf{x})=c w$. Then, see Figure 23 , when the triangle $t_{k}$ contains the edge $\left(w_{k}, v_{k}\right)$, our only choice is to add the edge $\left(v_{k}, z_{k}\right)$ (Case CI). When it contains the edge $\left(w_{k}, z_{k}\right)$, we can either add the edge 

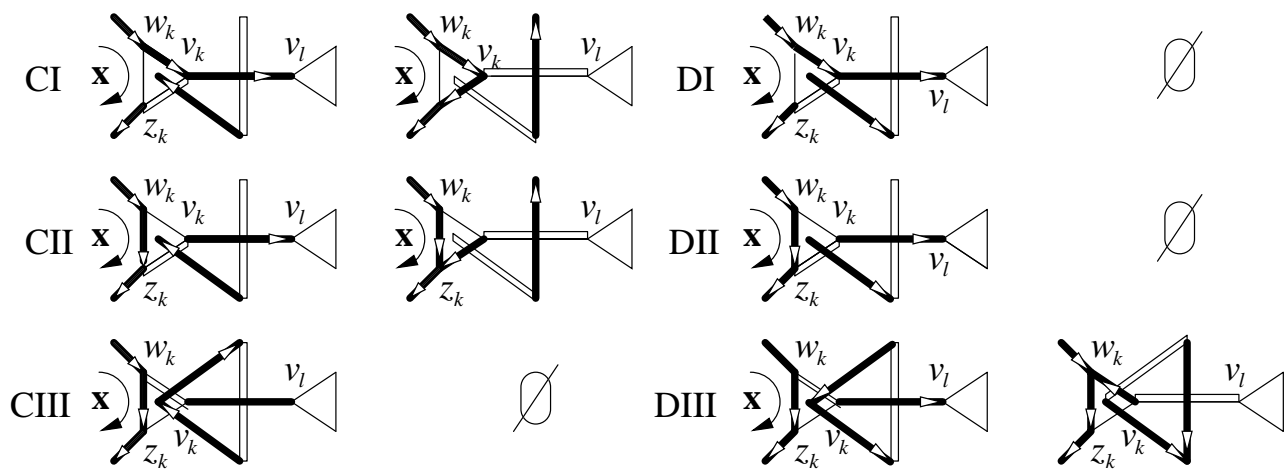

Case C

Case D

Figure 23: Removing the long edge $\left(v_{k}, v_{\ell}\right)$ when the dual of the edge $z_{k} v_{k}$ is oriented towards (away from) the triangle $t_{k}$ in Case C (Case D).

$\left(v_{k}, z_{k}\right)$ (Case CII) or the edge $\left(v_{k}, w_{k}\right)$ (Case CIII). We only allow this move when it is licit, that is in Cases CI and CII.

In Case $\mathrm{D}$, where the dual of the edge $z_{k} v_{k}$ is oriented away from the triangle $t_{k}$, a similar argument holds, and yields the last two columns of Figure 23, the move is only allowed in Case DIII. It is important to note that in each case, at most one move is allowed. By symmetry, if $\mathbf{x}$ has type $c c l w$, Cases $\mathrm{C}$ and $\mathrm{D}$ hold, with ' $w$ 's and ' $z$ 's exchanged.

Definition 6.3. When the above move is allowed, we refer to it as an essential reverse move, and denote by $\left(F, F^{*}\right)_{\{\cdot, \epsilon \mathbf{e}\}}=\left(F_{\{\cdot, \epsilon \mathbf{e}\}}, F_{\left\{\epsilon \mathbf{e}^{*}, \cdot\right\}}\right)$ the resulting pair of OCRSFs. When the move is not allowed, we set by convention $\left(F, F^{*}\right)_{\{\cdot, \epsilon \mathbf{e}\}}=\emptyset$.

The analogous of Proposition 25 also holds for essential reverse moves, and we do not write it out explicitly. The next lemma relates essential moves and reverse ones.

Lemma 26. Let $\left(F, F^{*}\right)$ be a pair of dual essential OCRSFs of $\mathcal{G}_{1}$ and $\mathcal{G}_{1}^{*}$, such that $F$ is compatible with $\mathbf{L}$ and $\tau$.

1. Let $\mathbf{e}$ be an unoriented edge of $E\left(G_{1}\right) \backslash \mathbf{L}$, and $\epsilon \in\{-,+\}$. Then, if an essential move can be performed on $(F, F)^{*}$, yielding a pair $\left(F, F^{*}\right)_{\{\epsilon \mathbf{e}, \cdot\}}$, the essential reverse primal/dual move, which removes the long edge $\epsilon \mathbf{e}$ from $\left(F, F^{*}\right)_{\{\epsilon \mathbf{e}, \cdot\}}$, can also be performed and:

$$
\left(\left(F, F^{*}\right)_{\{\epsilon \mathbf{e}, \cdot\}}\right)_{\{\cdot, \epsilon \mathbf{e}\}}=\left(F, F^{*}\right) .
$$

2. Let $\epsilon \mathbf{e}$ be an oriented edge of $\mathbf{L}$, where $\epsilon \in\{-,+\}$. Then, if an essential reverse primal/dual move can be performed on $\left(F, F^{*}\right)$, yielding a pair $\left(F, F^{*}\right)_{\{\cdot, \epsilon \mathbf{e}\}}$, the 
essential primal/dual move, which adds the long edge $\epsilon \mathbf{e}$ to $\left(F, F^{*}\right)_{\{\cdot, \epsilon \mathbf{e}\}}$, can also performed, and:

$$
\left(\left(F, F^{*}\right)_{\{\cdot, \epsilon \mathbf{e}\}}\right)_{\{\epsilon \mathbf{e}, \cdot\}}=\left(F, F^{*}\right) .
$$

Proof. From Figures 22 and 23, we immediately check that the moves AI/CI, AII/CII, BIII/DIII are inverse of eachother.

\subsubsection{Explicit construction}

Let $\left(\mathbf{F}, \mathbf{F}^{*}\right)$ be a pair of dual OCRSFs of $G_{1}$ and $G_{1}^{*}$, and let $\mathbf{e}_{1}, \cdots, \mathbf{e}_{m}$, be an arbitrary labeling of unoriented edges of $E\left(G_{1}\right) \backslash \mathbf{F}$. We let $J_{k}=\left\{\left(i_{1}, \cdots, i_{k}\right) \in\right.$ $\left.\{1, \cdots, m\}^{k} \mid 1 \leq i_{1}<\cdots<i_{k} \leq m\right\}$, with the convention that $J_{k}=\emptyset$, when $k=0$. Let $\tau \in\{c w, c c l w\}^{V\left(G_{1}\right)}$ be an assignment of types to vertices of $G_{1}$.

We now define by induction, for every $k \in\{0, \cdots, m\}$ and every $\left(i_{1}, \cdots, i_{k}\right) \in J_{k}$, the set,

$$
\mathcal{F}^{\tau,\left(\mathbf{F}, \mathbf{F}^{*}\right),\left\{\mathbf{e}_{i_{1}}, \cdots, \mathbf{e}_{i_{k}} ; \cdot\right\}}\left(\mathcal{G}_{1}, \mathcal{G}_{1}^{*}\right) .
$$

Initial step: $\mathcal{F}^{\tau,\left(\mathbf{F}, \mathbf{F}^{\prime}\right), \emptyset}\left(\mathcal{G}_{1}, \mathcal{G}_{1}^{*}\right)=\mathcal{F}^{\tau,\left(\mathbf{F}, \mathbf{F}^{*}\right)}\left(\mathcal{G}_{1}, \mathcal{G}_{1}^{*}\right)$, defined in Section 6.1.1.

Induction step: for $j=1, \cdots, k$,

$$
\begin{aligned}
& \mathcal{F}^{\tau,\left(\mathbf{F}, \mathbf{F}^{*}\right),\left\{\mathbf{e}_{i_{1}}, \cdots, \mathbf{e}_{i j} ; \cdot\right\}}\left(\mathcal{G}_{1}, \mathcal{G}_{1}^{*}\right)= \\
& \bigcup_{\left(F, F^{*}\right) \in \mathcal{F}^{\tau,\left(\mathbf{F}, \mathbf{F}^{*}\right),\left\{\mathbf{e}_{i_{1}}, \cdots, \mathbf{e}_{i_{j-1}} ; \cdot\right\}}}\left\{\left(F, F^{*}\right)_{\left\{+\mathbf{G}_{i_{j}}, \mathcal{G}_{1}^{*}\right)} \cup\left(F, F^{*}\right)_{\left\{-\mathbf{e}_{i_{j}}, \cdot\right\}}\right\},
\end{aligned}
$$

where $\left(F, F^{*}\right)_{\left\{ \pm \mathbf{e}_{i_{j}},\right\}}$ is defined in Section 6.1.2. Then, we have:

Proposition 27. The set $\mathcal{F}^{\tau,\left(\mathbf{F}, \mathbf{F}^{*}\right),\left\{\mathbf{e}_{i_{1}}, \cdots, \mathbf{e}_{i_{k}} ; \cdot\right\}}\left(\mathcal{G}_{1}, \mathcal{G}_{1}^{*}\right)$ is non empty, independent of the order in which edges are added, and consists of distinct pairs of essential dual OCRSFs of $\mathcal{G}_{1}$ and $\mathcal{G}_{1}^{*}$, compatible with $\tau$. Moreover, for every pair $\left(F, F^{*}\right)$ in this set,

1. either the reference number of $\left(F, F^{*}\right)$ in $\mathcal{G}_{1}$ is equal to the reference number of $(\mathbf{F}, \mathbf{F})^{*}$ in $G_{1}$, and:

$$
N_{\mathcal{G}_{1}, \mathcal{G}_{1}^{*}}\left(F, F^{*}\right)=N_{G_{1}, G_{1}^{*}}\left(\mathbf{F}, \mathbf{F}^{*}\right),
$$

2. or the reference numbers differ, and

$$
N_{\mathcal{G}_{1}, \mathcal{G}_{1}^{*}}\left(F, F^{*}\right)=N_{G_{1}, G_{1}^{*}}\left(\mathbf{F}, \mathbf{F}^{*}\right)=0 .
$$

Remark 28. Note that the set $\mathcal{F}^{\tau,\left(\mathbf{F}, \mathbf{F}^{*}\right),\left\{\mathbf{e}_{i_{1}}, \cdots, \mathbf{e}_{i_{k}} ; \cdot\right\}}\left(\mathcal{G}_{1}, \mathcal{G}_{1}^{*}\right)$ can be rewritten as:

$$
\bigcup_{\left(\epsilon_{i_{1}}, \cdots, \epsilon_{i_{k}}\right) \in\{-,+\}^{k}} \mathcal{F}^{\tau,\left(\mathbf{F}, \mathbf{F}^{*}\right),\left\{\epsilon_{i_{1}} \mathbf{e}_{i_{1}}, \cdots, \epsilon_{i_{k}} \mathbf{e}_{i_{k}} ; \cdot\right\}}\left(\mathcal{G}_{1}, \mathcal{G}_{1}^{*}\right),
$$


where $\mathcal{F}^{\tau,\left(\mathbf{F}, \mathbf{F}^{*}\right),\left\{\epsilon_{i_{1}} \mathbf{e}_{i_{1}}, \cdots, \epsilon_{i_{k}} \mathbf{e}_{i_{k}} ;\right\}}\left(\mathcal{G}_{1}, \mathcal{G}_{1}^{*}\right)$ is defined by induction as follows. The initial set is $\mathcal{F}^{\tau,\left(\mathbf{F}, \mathbf{F}^{*}\right)}\left(\mathcal{G}_{1}, \mathcal{G}_{1}^{*}\right)$, and for every $j \in\{1, \cdots, k\}$,

$$
\begin{aligned}
& \mathcal{F}^{\tau,\left(\mathbf{F}, \mathbf{F}^{*}\right),\left\{\epsilon_{i_{1}} \mathbf{e}_{i_{1}}, \cdots, \epsilon_{i_{j}} \mathbf{e}_{i_{j}} ; \cdot\right\}}\left(\mathcal{G}_{1}, \mathcal{G}_{1}^{*}\right) \\
& =\underset{\left(F, F^{*}\right) \in \mathcal{F}^{\tau,\left(\mathbf{F}, \mathbf{F}^{*}\right),\left\{\epsilon_{i_{1}} \mathbf{e}_{i_{1}}, \cdots, \epsilon_{i_{j-1}} \mathbf{e}_{i_{j-1}} ; \cdot\right\}}}{\bigcup_{\left(\mathcal{G}_{1}, \mathcal{G}_{1}^{*}\right)}}\left\{\left(F, F^{*}\right)_{\left\{\epsilon_{i_{j}} \mathbf{e}_{i_{j}}, \cdot\right\}}\right\} .
\end{aligned}
$$

Proof. As a consequence of Remark 28, it suffices to show that for every $\left(\epsilon_{i_{1}}, \cdots, \epsilon_{i_{k}}\right) \in$ $\{-,+\}^{k}$, the set $\mathcal{F}^{\tau,\left(\mathbf{F}, \mathbf{F}^{*}\right),\left\{\epsilon_{i_{1}} \mathbf{e}_{i_{1}}, \cdots, \epsilon_{i_{k}} \mathbf{e}_{i_{k}} ; \cdot\right\}}\left(\mathcal{G}_{1}, \mathcal{G}_{1}^{*}\right)$ satisfies Proposition 27. Let us first prove that the initial set $\mathcal{F}^{\tau,\left(\mathbf{F}, \mathbf{F}^{*}\right)}\left(\mathcal{G}_{1}, \mathcal{G}_{1}^{*}\right)$ satisfies all statements. By Lemma 22 and 23 , it is clearly non empty, and consists of pairs of essential dual OCRSFs of $\mathcal{G}_{1}$ and $\mathcal{G}_{1}^{*}$, compatible with $\tau$; and by Corollary 24, it satisfies Point 1.

Returning to the definition of essential moves and to the characterization of OCRSFs of $\mathcal{F}^{\tau,\left(\mathbf{F}, \mathbf{F}^{*}\right)}\left(\mathcal{G}_{1}, \mathcal{G}_{1}^{*}\right)$, we deduce that the set $\mathcal{F}^{\tau,\left(\mathbf{F}, \mathbf{F}^{*}\right),\left\{\epsilon_{i_{1}} \mathbf{e}_{i_{1}}, \cdots, \epsilon_{i_{k}} \mathbf{e}_{i_{k}} ; \cdot\right\}}\left(\mathcal{G}_{1}, \mathcal{G}_{1}^{*}\right)$ is also nonempty. By Proposition 25, we know that it consists of pairs of essential dual OCRSFs of $\mathcal{G}_{1}$ and $\mathcal{G}_{1}^{*}$ compatible with $\tau$. Moreover, since the orientation of edges not involved in the move remains unchanged, we deduce that the induction is in fact independent of the order in which edges are added. Proposition 25 also implies that either Point 1. or 2. is satisfied.

\section{Definition 6.4.}

$$
\begin{aligned}
\mathcal{S}^{\tau,\left(\mathbf{F}, \mathbf{F}^{*}\right)}\left(\mathcal{G}_{1}, \mathcal{G}_{1}^{*}\right) & =\bigcup_{k=0}^{m} \bigcup_{\left\{\left\{\mathbf{e}_{i_{1}}, \cdots, \mathbf{e}_{i_{k}}\right\}:\left(i_{1}, \cdots, i_{k}\right) \in J_{k}\right\}} \mathcal{F}^{\tau,\left(\mathbf{F}, \mathbf{F}^{*}\right),\left\{\mathbf{e}_{i_{1}}, \cdots, \mathbf{e}_{i} ; \cdot\right\}}\left(\mathcal{G}_{1}, \mathcal{G}_{1}^{*}\right) \\
\mathcal{S}^{\left(\mathbf{F}, \mathbf{F}^{*}\right)}\left(\mathcal{G}_{1}, \mathcal{G}_{1}^{*}\right) & =\bigcup_{\tau \in\{c w, c c w l\}^{V\left(G_{1}\right)}} \mathcal{S}^{\tau,\left(\mathbf{F}, \mathbf{F}^{*}\right)}\left(\mathcal{G}_{1}, \mathcal{G}_{1}^{*}\right)
\end{aligned}
$$

The next theorem proves that we exactly obtain all pairs of dual CRSFs of $\mathcal{G}_{1}$ and $\mathcal{G}_{1}^{*}$.

\section{Theorem 29.}

1. $\bigcup_{\left(\mathbf{F}, \mathbf{F}^{*}\right) \in \mathcal{F}\left(G_{1}, G_{1}^{*}\right)} \mathcal{S}^{\left(\mathbf{F}, \mathbf{F}^{*}\right)}\left(\mathcal{G}_{1}, \mathcal{G}_{1}^{*}\right)=\mathcal{F}^{0}\left(\mathcal{G}_{1}, \mathcal{G}_{1}^{*}\right)$.

2. When $\left(\mathbf{F}_{1}, \mathbf{F}_{1}^{*}\right) \neq\left(\mathbf{F}_{2}, \mathbf{F}_{2}^{*}\right)$, then $\mathcal{S}^{\left(\mathbf{F}_{1}, \mathbf{F}_{1}^{*}\right)}\left(\mathcal{G}_{1}, \mathcal{G}_{1}^{*}\right) \cap \mathcal{S}^{\left(\mathbf{F}_{2}, \mathbf{F}_{2}^{*}\right)}\left(\mathcal{G}_{1}, \mathcal{G}_{1}^{*}\right)=\emptyset$.

Proof. The inclusion $\subset$ of Point 1. is a direct consequence of Proposition 27.

Consider a pair $\left(F, F^{*}\right)$ of dual essential OCRSFs of $\mathcal{G}_{1}$ and $\mathcal{G}_{1}^{*}$, and let us show that there exists a pair of dual OCRSFs $\left(\mathbf{F}, \mathbf{F}^{*}\right)$ of $G_{1}$ and $G_{1}^{*}$, such that $\left(F, F^{*}\right) \in$ $\mathcal{S}^{\left(\mathbf{F}, \mathbf{F}^{*}\right)}\left(\mathcal{G}_{1}, \mathcal{G}_{1}^{*}\right)$. By definition of essential OCRSFs of $\mathcal{G}_{1}$, there exists a subset of edges $\mathbf{L}$ of $\mathcal{L}$, and $\tau \in\{c w, c c l w\}^{V\left(G_{1}\right)}$, such that $F$ is an essential OCRSF compatible with $\mathbf{L}$ and $\tau$, and $F^{*}$ is a dual OCRSF of $F$. Recall that the set $\mathbf{L}$ defines, for every decoration 
$\mathbf{x}$, a set of root vertices $R_{\mathbf{x}}(\mathbf{L})$. Let us fix a decoration $\mathbf{x}$, assume that $\tau(\mathbf{x})=c w$ (the case where $\tau(\mathbf{x})=$ cclw being similar), and omit the argument $\mathbf{x}$ in the sequel. By Corollary 15, the restriction of $F$ to the decoration $\mathbf{x}$ consists of:

- all inner edges oriented clockwise,

- one of the three possible 2-edge configuration at the triangle of every non-root vertex, with the appropriate orientation,

- one of the two following 1-edge configurations at the triangle of every root vertex $v_{i} \in R_{\mathbf{x}}(\mathbf{L})$ :

$$
\left\{\left(w_{i}, v_{i}\right)\right\},\left\{\left(w_{i}, z_{i}\right)\right\}
$$

with the additional constraint that the triangle of at least one root vertex contains the configuration $\left(w_{i}, v_{i}\right)$.

Let us now study properties of the dual OCRSF $F^{*}$ at the decoration $\mathbf{x}$, see also Figure 24. Denote by $c^{*}$ the dual vertex at the center of the decoration, and by $t_{1}^{*}, \cdots, t_{d_{\mathbf{x}}}^{*}$ the dual vertices at the center of the triangles $t_{1}, \cdots, t_{d_{\mathbf{x}}}$. As inner edges are always present in the OCRSF $F$, we omit their dual edges in our representation of the dual graph.

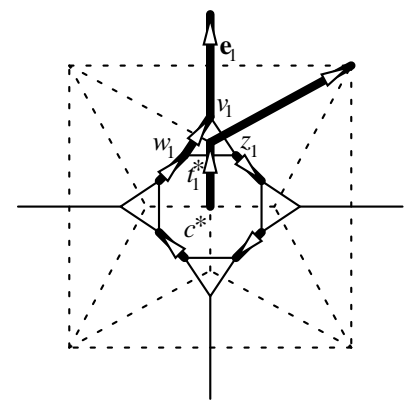

Figure 24: The restriction of $F$ and $F^{*}$ to a decoration.

Since $F^{*}$ is an OCRSF, there is exactly one edge $\left(c^{*}, t_{i}^{*}\right)$ exiting the vertex $c^{*}$, we set by convention $i=1$. Then, since there must also be one edge exiting the vertex $t_{1}^{*}$, this means that $F$ must contain the edge $\left(w_{1}, v_{1}\right)$, implying that $v_{1}$ is a root vertex of $\mathbf{L}$, and that the long edge $\epsilon_{1} \mathbf{e}_{1}$, where $\epsilon_{1} \in\{-,+\}$, whose initial vertex is $v_{1}$, belongs to $\mathbf{L}$. Repeating this for every decoration of $\mathcal{G}_{1}$ defines a subset of oriented edges $\mathbf{F}$ of $G_{1}$,

$$
\mathbf{F}=\bigcup_{\mathbf{x} \in V\left(G_{1}\right)}\left\{\epsilon_{1}(\mathbf{x}) \mathbf{e}_{1}(\mathbf{x})\right\}
$$

such that every vertex of $G_{1}$ has exactly one outgoing edge of this subset.

Suppose that the decoration $\mathbf{x}$ has another root vertex $v_{i}(i \neq 1)$, and let $\epsilon_{i} \mathbf{e}_{i}$ be the edge of $\mathbf{L}$ whose initial vertex is $v_{i}$. Then, at the triangle $t_{i}$, the OCRSF $F$ consists of: 
- either the edge $\left(w_{i}, z_{i}\right)$; then, the dual of the edges $w_{i} v_{i}, z_{i} v_{i}$ belong to $F^{*}$. A priori, there are two possible orientations for the dual edges, and which one it is is fixed by $F^{*}$. In each of the two cases, either the essential reverse move CII or DIII can be performed on the pair $\left(F, F^{*}\right)$, yielding a pair $\left(F, F^{*}\right)_{\left\{\cdot, \epsilon_{i} \mathbf{e}_{i}\right\}}$ of dual essential OCRSFs of $\mathcal{G}_{1}$ and $\mathcal{G}_{1}^{*}$.

- or the edge $\left(w_{i}, v_{i}\right)$; then, the dual edge $\left(t_{i}^{*}, c^{*}\right)$ belongs to $F^{*}$ and must be oriented towards $c^{*}$. Indeed, otherwise the vertex $c^{*}$ would have two exiting edges of $F^{*}$ which contradicts the fact of being an OCRSF. This implies that the dual of the edge $z_{i} v_{i}$, which also belongs to $F^{*}$ is oriented towards $t_{i}^{*}$. As a consequence, the essential reverse move CI can be performed on the pair $\left(F, F^{*}\right)$, yielding a pair $\left(F, F^{*}\right)_{\left\{\cdot, \epsilon_{i} \mathbf{e}_{i}\right\}}$ of dual essential OCRSFs of $\mathcal{G}_{1}$ and $\mathcal{G}_{1}^{*}$.

Since the orientation of edges not involved in the move remains unchanged by essential reverse moves, we can repeat this for every root vertex different from $v_{1}$ of the decoration $\mathbf{x}$, and for every decoration $\mathbf{x}$ of $\mathcal{G}_{1}$. By the analogous of Proposition 25 for essential reverse moves, this yields a pair $\left(\bar{F}, \bar{F}^{*}\right)$ of dual essential OCRSFs of $\mathcal{G}_{1}$ and $\mathcal{G}_{1}^{*}$, such that $\bar{F}$ is compatible with $\mathbf{F}$ and $\tau$. Then, by Lemma 22, this implies that $\mathbf{F}$ is an OCRSF of $G_{1}$. Let $\mathbf{F}^{*}$ be the restriction to dual long edges of $\bar{F}^{*}$. Then, by Lemma 23 , $\mathbf{F}^{*}$ is a dual OCRSF of $\mathbf{F}$, and we deduce that:

$$
\left(\bar{F}, \bar{F}^{*}\right) \in \mathcal{F}^{\tau,\left(\mathbf{F}, \mathbf{F}^{*}\right)}\left(\mathcal{G}_{1}, \mathcal{G}_{1}^{*}\right) .
$$

By Lemma 26, if an essential reverse move can be performed on a pair of dual OCRSFs, removing an oriented long edge, then the essential move adding the same long edge can be performed to recover the original pair. Applying this recursively, we deduce that:

$$
\left(F, F^{*}\right) \in \mathcal{F}^{\tau,\left(\mathbf{F}, \mathbf{F}^{*}\right),\{\mathbf{L} \backslash \mathbf{F}\}}\left(\mathcal{G}_{1}, \mathcal{G}_{1}^{*}\right) \subset \mathcal{S}^{\left(\mathbf{F}, \mathbf{F}^{*}\right)}\left(\mathcal{G}_{1}, \mathcal{G}_{1}^{*}\right),
$$

thus proving Point 1. of Theorem 29.

Let us now prove Point 2. Suppose that there are two distinct pairs $\left(\mathbf{F}_{1}, \mathbf{F}_{1}^{*}\right)$ and $\left(\mathbf{F}_{2}, \mathbf{F}_{2}^{*}\right)$ of dual OCRSFs of $G_{1}$ and $G_{1}^{*}$, such that:

$$
\left(F, F^{*}\right) \in \mathcal{S}^{\left(\mathbf{F}_{1}, \mathbf{F}_{1}^{*}\right)}\left(\mathcal{G}_{1}, \mathcal{G}_{1}^{*}\right) \cap \mathcal{S}^{\left(\mathbf{F}_{2}, \mathbf{F}_{2}^{*}\right)}\left(\mathcal{G}_{1}, \mathcal{G}_{1}^{*}\right)
$$

Let us return to properties of $\left(F, F^{*}\right)$ at a fixed decoration $\mathbf{x}$ of $\mathcal{G}_{1}$. In $F^{*}$, the dual edge of the edge $z_{1} v_{1}$ is oriented away from $t_{1}^{*}$. Now, suppose that the edge $\epsilon_{1} \mathbf{e}_{1}$ is added by an essential move. This implies that in the dual graph, the dual of the edge $z_{1} v_{1}$ must be added. Referring to Figure 22, which describes possible essential moves, we see that this dual edge is then oriented towards $t_{1}^{*}(\mathbf{x})$, which is a contradiction. Thus, $\epsilon_{1} \mathbf{e}_{1}$ must be an edge of the original OCRSF. Since essential moves do not change the orientation of edges not involved in the move, we repeat this argument for every decoration, and deduce that:

$$
\mathbf{F}_{1}=\mathbf{F}_{2}=\mathbf{F}
$$


where $\mathbf{F}$ is defined in Equation (11). As a consequence $\left(F, F^{*}\right) \in \mathcal{F}^{\tau,\left(\mathbf{F}, \mathbf{F}_{1}^{*}\right),\{\mathbf{L} \backslash \mathbf{F}\}}\left(\mathcal{G}_{1}, \mathcal{G}_{1}^{*}\right) \cap$ $\mathcal{F}^{\tau,\left(\mathbf{F}, \mathbf{F}_{2}^{*}\right),\{\mathbf{L} \backslash \mathbf{F}\}}\left(\mathcal{G}_{1}, \mathcal{G}_{1}^{*}\right)$, where $\mathbf{F}_{1}^{*}$ and $\mathbf{F}_{2}^{*}$ are two distinct dual OCRSFs of $\mathbf{F}$. This means that there exists $\left(F_{1}, F_{1}^{*}\right) \in \mathcal{F}^{\tau,\left(\mathbf{F}, \mathbf{F}_{1}^{*}\right)}\left(\mathcal{G}_{1}, \mathcal{G}_{1}^{*}\right)$ and $\left(F_{2}, F_{2}^{*}\right) \in \mathcal{F}^{\tau,\left(\mathbf{F}, \mathbf{F}_{2}^{*}\right)}\left(\mathcal{G}_{1}, \mathcal{G}_{1}^{*}\right)$, such that $\left(F, F^{*}\right)$ is obtained from each of $\left(F_{i}, F_{i}\right)^{*}$ by successively adding the same set of oriented long edges $\mathbf{L} \backslash \mathbf{F}$ with essential moves. By Part 1. of Lemma 27, if an essential move can be performed to add an oriented long edge, then the reverse move can also be performed to recover the original pair. Moreover, looking at Figure 9, describing possible essential reverse moves, we see that there is at most one essential reverse move for removing a given oriented long edge. Thus, the same essential reverse moves are performed on $\left(F, F^{*}\right)$ to recover $\left(F_{1}, F_{1}^{*}\right)$ and $\left(F_{2}, F_{2}^{*}\right)$, thus implying that this is in fact the same pair. Since $\mathbf{F}_{i}^{*}$ is the restriction to dual long edges of $F_{i}^{*}(i=1,2)$, we deduce that $\mathbf{F}_{1}^{*}=\mathbf{F}_{2}^{*}$.

\subsection{The construction is weight preserving}

In this section, we state and prove Theorem 30, which establishes that the construction is weight preserving: we show that the sum of the weights of all pairs of essential dual OCRSFs of $\mathcal{G}_{1}$ and $\mathcal{G}_{1}^{*}$ in $\mathcal{S}^{\left(\mathbf{F}, \mathbf{F}^{*}\right)}\left(\mathcal{G}_{1}, \mathcal{G}_{1}^{*}\right)$ is equal, up to a constant which only depends on the graph $G_{1}$, to the weight of the pair of dual OCRSFs $\left(\mathbf{F}, \mathbf{F}^{*}\right)$ of $G_{1}$ and $G_{1}^{*}$. As a consequence we recover, by an explicit computation, Theorem 2 of [BdT10b], see Corollary 31. Note that the constant could not be explicited in the proof of Theorem 2 , but could only be recovered a posteriori in [BdT10a].

Theorem 30. Let $\left(\mathbf{F}, \mathbf{F}^{*}\right)$ be a pair of dual OCRSFs of $G_{1}$ and $G_{1}^{*}$. Then,

$$
\begin{aligned}
\sum_{\left(F, F^{*}\right) \in \mathcal{S}^{\left(\mathbf{F}, \mathbf{F}^{*}\right)}}\left(\prod_{\left.\mathcal{G}_{1}, \mathcal{G}_{1}^{*}\right)} f_{x} \overline{f_{y}} K_{x, y}\right) & \left(-\mathbf{z}^{h_{0}(F)} \mathbf{w}^{v_{0}(F)}\right)^{\frac{1}{2} N\left(F, F^{*}\right)}= \\
& =\mathbf{C}\left(\prod_{(\mathbf{x}, \mathbf{y}) \in \mathbf{F}} \tan \theta_{\mathbf{x y}}\right)\left(-\mathbf{z}^{h_{0}(\mathbf{F})} \mathbf{w}^{v_{0}(\mathbf{F})}\right)^{\frac{1}{2} N\left(\mathbf{F}, \mathbf{F}^{*}\right)},
\end{aligned}
$$

where $\mathbf{C}=2^{4\left|E\left(G_{1}\right)\right|+\left|V\left(G_{1}\right)\right|} \prod_{\mathbf{x y} \in E\left(G_{1}\right)} \sin ^{2}\left(\frac{\theta_{\mathbf{x y}}}{2}\right) \cos \theta_{\mathbf{x y}}$.

Proof. Using notations introduced in Section 6.1.3, let us recall that:

$$
\mathcal{S}^{\left(\mathbf{F}, \mathbf{F}^{*}\right)}\left(\mathcal{G}_{1}, \mathcal{G}_{1}^{*}\right)=\bigcup_{\tau \in\{c w, c c l w\}} \bigcup_{k=0}^{m} \bigcup_{\left\{\left\{\mathbf{e}_{i_{1}}, \cdots, \mathbf{e}_{i_{k}}\right\}:\left(i_{1}, \cdots, i_{k}\right) \in J_{k}\right\}} \mathcal{F}^{\tau,\left(\mathbf{F}, \mathbf{F}^{*}\right),\left\{\mathbf{e}_{i_{1}}, \cdots, \mathbf{e}_{i_{k}}\right\}}\left(\mathcal{G}_{1}, \mathcal{G}_{1}^{*}\right)
$$

By Proposition 27, we know that for every pair $\left(F, F^{*}\right)$ of dual essential OCRSFs of $\mathcal{S}^{\left(\mathbf{F}, \mathbf{F}^{*}\right)}\left(\mathcal{G}_{1}, \mathcal{G}_{1}^{*}\right)$ :

$$
\left(-\mathbf{z}^{h_{0}(F)} \mathbf{w}^{v_{0}(F)}\right)^{\frac{1}{2} N\left(F, F^{*}\right)}=\left(-\mathbf{z}^{h_{0}(\mathbf{F})} \mathbf{w}^{v_{0}(\mathbf{F})}\right)^{\frac{1}{2} N\left(\mathbf{F}, \mathbf{F}^{*}\right)},
$$


so that we only need to handle the weights which do not involve the coefficients $\mathrm{z}$ and w. Consider $\tau \in\{c w, c c l w\}^{V\left(G_{1}\right)}$, a fixed assignment of types to vertices of $G_{1}$, and let us denote by $\mathcal{W}_{k}$ the weighted sum (excluding $\mathbf{z}$ and $\mathrm{w}$ ) of configurations of $\mathcal{F}^{\tau,\left(\mathbf{F}, \mathbf{F}^{*}\right),\left\{\mathbf{e}_{i_{1}}, \cdots, \mathbf{e}_{i_{k}}\right\}}\left(\mathcal{G}_{1}, \mathcal{G}_{1}^{*}\right)$. We now compute $\mathcal{W}_{k}$ by induction on $k$.

In all computations below, we use the definition of the Kasteleyn orientation of Section 3.1, the definition of the function $\left(f_{x}\right)_{x \in V\left(\mathcal{G}_{1}\right)}$ and of the rhombus half-angles in $\mathbb{R} / 4 \pi \mathbb{Z}$ of Section 4.2.1.

Computation of $\mathcal{W}_{0}$. Recall that $\mathcal{F}^{\tau,\left(\mathbf{F}, \mathbf{F}^{*}\right)}\left(\mathcal{G}_{1}, \mathcal{G}_{1}^{*}\right)$ consists of all pairs $\left(F, F^{*}\right)$ of dual OCRSFs of $\mathcal{G}_{1}$ and $\mathcal{G}_{1}^{*}$, such that $F$ is an essential OCRSF compatible with $\mathbf{F}$ and $\tau$, characterized in Lemma 22, and $F^{*}$ is the unique dual OCRSF whose restriction to dual long edges is $\mathbf{F}^{*}$. Recall also that, for every decoration $\mathbf{x}$ of $\mathcal{G}_{1}, v_{1}(\mathbf{x})$ denotes the unique root vertex induced by $\mathbf{F}$.

1. Contribution of long edges of $\mathbf{F}$. Let $(\mathbf{x}, \mathbf{y})$ be an oriented edge of $\mathbf{F}$, and let

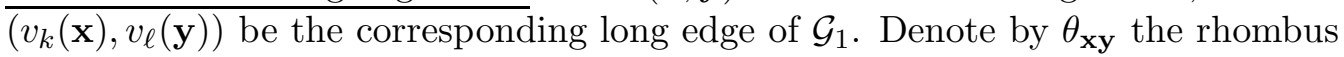
half-angle of $\mathbf{x y}$. Then the contribution of this edge to $\mathcal{W}_{0}$ is:

$$
f_{v_{k}} \overline{f_{v_{\ell}}} K_{v_{k}, v_{\ell}} .
$$

By definition of the Kasteleyn matrix $K$, and of the vectors $f$, we have:

$$
\begin{aligned}
& K_{v_{k}, v_{\ell}}=\varepsilon_{v_{k}, v_{\ell}} \cot \frac{\theta_{\mathbf{x y}}}{2} \\
& f_{v_{k}}=e^{-i \frac{\alpha_{w_{k}}}{2}}-e^{-i \frac{\alpha_{z_{k}}}{2}}, \quad f_{v_{\ell}}=e^{-i \frac{\alpha_{w_{\ell}}}{2}}-e^{-i \frac{\alpha_{z_{\ell}}}{2}} .
\end{aligned}
$$

Moreover, by definition of the rhombus half-angles in $\mathbb{R} / 4 \pi \mathbb{Z}$ :

$$
f_{v_{\ell}}=i \varepsilon_{v_{k}, v_{\ell}} f_{v_{k}} \text {, }
$$

so that $\overline{f_{v_{\ell}}}=-i \varepsilon_{v_{k}, v_{\ell}} \overline{f_{v_{k}}}$. As a consequence:

$$
\begin{aligned}
& f_{v_{k}} \overline{f_{v_{\ell}}} K_{v_{k}, v_{\ell}}=-i \cot \frac{\theta_{\mathbf{x y}}}{2}\left|f_{v_{k}}\right|^{2}=-i \cot \frac{\theta_{\mathbf{x y}}}{2} 2\left(1-\cos \theta_{\mathbf{x y}}\right) \\
& =-2 i \sin \theta_{\mathbf{x y}} \text {. }
\end{aligned}
$$

2. Contribution of inner edges of decorations. Let $\mathbf{x}$ be a decoration of $\mathcal{G}_{1}$, and let $w_{j}(\mathbf{x}) z_{j+1}(\mathbf{x})$ be a a generic inner edge of $\mathbf{x}$.

○ If $\tau(\mathbf{x})=c w$, then the edge is oriented from $z_{j+1}$ to $w_{j}$, and its contribution to $\mathcal{W}_{0}$ is:

$$
\varepsilon_{z_{j+1}, w_{j}} f_{z_{j+1}} \overline{f_{w_{j}}} .
$$

Moreover, by definition of the angles in $\mathbb{R} / 4 \pi \mathbb{Z}$, we have $f_{z_{j+1}}=-\varepsilon_{w_{j}, z_{j+1}} f_{w_{j}}$, so that the contribution is:

$$
-\varepsilon_{z_{j+1}, w_{j}} \varepsilon_{w_{j}, z_{j+1}} f_{w_{j}} \overline{f_{w_{j}}}=1 .
$$


$\circ$ If $\tau(\mathbf{x})=c c l w$, then the edge is oriented from $w_{j}$ to $z_{j+1}$, and its contribution to $\mathcal{W}_{0}$ is:

$$
\varepsilon_{w_{j}, z_{j+1}} f_{w_{j}} \overline{f_{z_{j+1}}}=-1
$$

3. Contribution of triangles of root vertices. Let $\mathbf{x}$ be a decoration of $\mathcal{G}_{1}$, and $t_{1}(\mathbf{x})$ be the triangle of the root vertex $v_{1}(\mathbf{x})$. Denote by $\theta_{1}(\mathbf{x})$ the rhombus half-angle of the long edge incident to $v_{1}(\mathbf{x})$.

○ If $\tau(\mathbf{x})=c w$, then the triangle $t_{1}$ contains the edge $\left(w_{1}, v_{1}\right)$, so that its contribution to $\mathcal{W}_{0}$ is:

$$
f_{w_{1}} \overline{f_{v_{1}}} K_{w_{1}, v_{1}}=e^{-i \frac{\alpha_{w_{1}}}{2}}\left(e^{i \frac{\alpha_{w_{1}}}{2}}-e^{i \frac{\alpha z_{1}}{2}}\right)=1-e^{-i \theta_{1}}=2 i \sin \left(\frac{\theta_{1}}{2}\right) e^{-i \frac{\theta_{1}}{2}} .
$$

○ If $\tau(\mathbf{x})=c c l w$, then the triangle $t_{1}$ contains the edge $\left(z_{1}, v_{1}\right)$, so that its contribution to $\mathcal{W}_{0}$ is:

$$
f_{z_{1}} \overline{f_{v_{1}}} K_{z_{1}, v_{1}}=e^{-i \frac{\alpha z_{1}}{2}}\left(e^{i \frac{\alpha w_{1}}{2}}-e^{i \frac{\alpha z_{1}}{2}}\right)=-1+e^{i \theta_{1}}=2 i \sin \left(\frac{\theta_{1}}{2}\right) e^{i \frac{\theta_{1}}{2}}
$$

4. Contribution of triangles of non-root vertices. Let $\mathbf{x}$ be a decoration of $\mathcal{G}_{1}, t_{j}(\mathbf{x})$ $(j \neq 1)$ be a triangle of a non-root vertex, and $\theta_{j}$ be the rhombus half-angle of the long edge incident to $v_{j}(\mathbf{x})$. Then, $t_{j}(\mathbf{x})$ contains any of the three possible 2-edge configurations, with the orientation induced by the type. Thus,

○ If $\tau(\mathbf{x})=c w$, its contribution to $\mathcal{W}_{0}$ is, see also Figure 9 :

$$
\begin{aligned}
& f_{w_{j}} \overline{f_{v_{j}}} K_{w_{j}, v_{j}} f_{v_{j}} \overline{f_{z_{j}}} K_{v_{j}, z_{j}}+f_{v_{j}} \overline{f_{z_{j}}} K_{v_{j}, z_{j}} f_{w_{j}} \overline{f_{z_{j}}} K_{w_{j}, z_{j}}+f_{v_{j}} \overline{f_{w_{j}}} K_{v_{j}, w_{j}} f_{w_{j}} \overline{f_{z_{j}}} K_{w_{j}, z_{j}}= \\
& =f_{w_{j}} \overline{f_{z_{j}}} f_{v_{j}}\left(\overline{f_{v_{j}}}-\overline{f_{z_{j}}}+\overline{f_{w_{j}}}\right) \\
& =f_{w_{j}} \overline{f_{z_{j}}} f_{v_{j}}\left(2 \overline{f_{w_{j}}}\right) \quad(\text { by definition of the vector } f) \\
& =2 \overline{f_{z_{j}}} f_{v_{j}}=-2 e^{i \frac{\alpha z_{j}}{2}}\left(e^{-i \frac{\alpha w_{j}}{2}}-e^{-i \frac{\alpha z_{j}}{2}}\right)=2\left(1-e^{-i \theta_{j}}\right) \\
& =4 i \sin \left(\frac{\theta_{j}}{2}\right) e^{-i \frac{\theta_{j}}{2}}
\end{aligned}
$$

○ If $\tau(\mathbf{x})=c c l w$, its contribution to $\mathcal{W}_{0}$ is:

$$
\begin{aligned}
(-1) f_{v_{j}} \overline{f_{w_{j}}} f_{z_{j}} \overline{f_{w_{j}}}+f_{v_{j}} \overline{f_{z_{j}}} f_{z_{j}} \overline{f_{w_{j}}}+(-1) f_{z_{j}} \overline{f_{v_{j}}}(-1) f_{v_{j}} \overline{f_{w_{j}}}= \\
=\overline{f_{w_{j}}} f_{z_{j}} f_{v_{j}}\left(-\overline{f_{w_{j}}}+\overline{f_{z_{j}}}+\overline{f_{v_{j}}}\right) \\
=\overline{f_{w_{j}}} f_{z_{j}} f_{v_{j}}\left(2 \overline{f_{z_{j}}}\right) \quad(\text { by definition of the vectors } f) \\
=2 \overline{f_{w_{j}}} f_{v_{j}}=2 e^{i \frac{\alpha w_{j}}{2}}\left(e^{-i \frac{\alpha w_{j}}{2}}-e^{-i \frac{\alpha z_{j}}{2}}\right)=2\left(1-e^{i \theta_{j}}\right) \\
=-4 i \sin \left(\frac{\theta_{j}}{2}\right) e^{i \frac{\theta_{j}}{2}}
\end{aligned}
$$


Combining this, we deduce the contribution of a decoration $\mathbf{x}$. Note that in computations below, we use the fact that $\sum_{j=1}^{d_{\mathbf{x}}} \frac{\theta_{j}}{2}=\frac{\pi}{2}$.

○ If $\tau(\mathbf{x})=c w$, the contribution of a decoration $\mathbf{x}$ to $\mathcal{W}_{0}$ is:

$$
\left(\prod_{j=1}^{d_{\mathbf{x}}} 2 i \sin \left(\frac{\theta_{j}}{2}\right) e^{-i \frac{\theta_{j}}{2}}\right) 2^{d_{\mathbf{x}}-1}=-2^{2 d_{\mathbf{x}}-1} i^{d_{\mathbf{x}}+1} \prod_{j=1}^{d_{\mathbf{x}}} \sin \left(\frac{\theta_{j}}{2}\right) .
$$

○ If $\tau(\mathbf{x})=c c l w$, the contribution of a decoration $\mathbf{x}$ to $\mathcal{W}_{0}$ is:

$$
\left(\prod_{j=1}^{d_{\mathbf{x}}}(-1) 2 i \sin \left(\frac{\theta_{j}}{2}\right) e^{i \frac{\theta_{j}}{2}}\right)(-2)^{d_{\mathbf{x}}-1}=-2^{2 d_{\mathbf{x}}-1} i^{d_{\mathbf{x}}+1} \prod_{j=1}^{d_{\mathbf{x}}} \sin \left(\frac{\theta_{j}}{2}\right) .
$$

We deduce that the contribution of a decoration is in fact independent of its type. Let us denote by

$$
N=V\left(G_{1}\right), M=E\left(G_{1}\right) .
$$

Then, since $\mathbf{F}$ is an OCRSF of $G_{1}$, it has $N$ edges. Taking the product over all long edges of $\mathbf{F}$ yields a contribution:

$$
(-2)^{N} i^{N} \prod_{(\mathbf{x}, \mathbf{y}) \in \mathbf{F}} \sin \theta_{\mathbf{x y}}
$$

where the contribution is in fact independent of the orientation of the edges. Observing that $\sum_{\mathbf{x} \in V\left(G_{1}\right)} d_{\mathbf{x}}=2 M$, and taking the product over all decorations of $\mathcal{G}_{1}$ yields a contribution:

$$
(-1)^{N} 2^{4 M-N} i^{2 M+N} \prod_{\mathbf{x y} \in E\left(G_{1}\right)} \sin ^{2}\left(\frac{\theta_{\mathbf{x y}}}{2}\right) .
$$

Taking the product of Equations (15) and (16), gives:

$$
\mathcal{W}_{0}=\left[2^{4 M}(-1)^{M+N} \prod_{\mathbf{x y} \in E\left(G_{1}\right)} \sin ^{2}\left(\frac{\theta_{\mathbf{x y}}}{2}\right)\right] \prod_{\mathbf{x y} \in \mathbf{F}} \sin \theta_{\mathbf{x y}} .
$$

Computation of $\mathcal{W}_{k}, k \geq 1$. Recall that $\mathcal{F}^{\tau,\left(\mathbf{F}, \mathbf{F}^{*}\right),\left\{\mathbf{e}_{i_{1}}, \cdots, \mathbf{e}_{i_{k}}\right\}}\left(\mathcal{G}_{1}, \mathcal{G}_{1}^{*}\right)$ consists of all pairs $\left(F, F^{*}\right)$ of dual essential OCRSFs of $\mathcal{G}_{1}$ and $\mathcal{G}_{1}^{*}$, obtained by performing essential moves adding the edge $+\mathbf{e}_{i_{k}}$ or $-\mathbf{e}_{i_{k}}$, on pairs of dual OCRSFs of $\mathcal{F}^{\tau,\left(\mathbf{F}, \mathbf{F}^{*}\right),\left\{\mathbf{e}_{i_{1}}, \cdots, \mathbf{e}_{i_{k-1}}\right\}}\left(\mathcal{G}_{1}, \mathcal{G}_{1}^{*}\right)$. Using Lemma 22, characterizing the set $\mathcal{F}^{\left.\tau,\left(\mathbf{F}, \mathbf{F}^{*}\right)\right\}}\left(\mathcal{G}_{1}, \mathcal{G}_{1}^{*}\right)$, and returning to the definition of essential moves, see Figure 22, we deduce by induction that, for every $k \in\{0, \cdots, m\}$ and for every $\left(i_{1}, \cdots, i_{k}\right) \in J_{k}$, the restriction to a decoration $\mathbf{x}$ of $\mathcal{G}_{1}$ of the first component $F$ of a pair $\left(F, F^{*}\right)$ of dual OCRSFs of $\mathcal{F}^{\tau,\left(\mathbf{F}, \mathbf{F}^{*}\right),\left\{\mathbf{e}_{i_{1}}, \cdots, \mathbf{e}_{i_{k}}\right\}}\left(\mathcal{G}_{1}, \mathcal{G}_{1}^{*}\right)$ contains:

1. all inner edges with the orientation induced by the type $\tau(\mathbf{x})$, 
2. any of the three possible 2-edge configurations at triangles of non-root vertices,

3. the edge $\left(w_{1}, v_{1}\right)\left(\operatorname{resp} .\left(z_{1}, v_{1}\right)\right)$ at the triangle $t_{1}(\mathbf{x})$, if $\tau(\mathbf{x})=c w(\operatorname{resp} . \tau(\mathbf{x})=$ $c c l w)$

4. one or two of the 1-edge configurations at triangles of other root vertices, depending on whether one or two essential moves can be performed.

Let us compute the ratio $\frac{\mathcal{W}_{k}}{\mathcal{W}_{k-1}}$. To simplify notations, we denote by $\mathbf{x y}$ the edge $\mathbf{e}_{i_{k}}$, by $v_{k}(\mathbf{x}) v_{\ell}(\mathbf{y})$ the corresponding long edge of $\mathcal{G}_{1}$, and by $\theta$ the corresponding rhombus half-angle.

The edge $\mathbf{e}_{i_{k}}$ is absent in OCRSFs of $\mathcal{F}^{\tau,\left(\mathbf{F}, \mathbf{F}^{*}\right),\left\{\mathbf{e}_{i_{1}}, \cdots, \mathbf{e}_{i_{k-1}}\right\}}\left(\mathcal{G}_{1}, \mathcal{G}_{1}^{*}\right)$, so that $v_{k}$ and $v_{\ell}$ are non-root vertices. By Point 3., this implies that the triangles $t_{k}$ and $t_{\ell}$ each contain any of the three possible 2-edge configurations. Using equations (13) and (14), we deduce that the contribution to $\mathcal{W}_{k-1}$ of the absent edge and of the two incident triangles is:

- If $\tau(\mathbf{x})=c w$ and $\tau(\mathbf{y})=c w$,

$$
\left(4 i \sin \left(\frac{\theta}{2}\right) e^{-i \frac{\theta}{2}}\right)^{2}=-16 \sin ^{2}\left(\frac{\theta}{2}\right) e^{-i \theta} .
$$

- If $\tau(\mathbf{x})=c w$ and $\tau(\mathbf{y})=c c l w$, or $\tau(\mathbf{x})=c c l w$ and $\tau(\mathbf{y})=c w$,

$$
16 \sin ^{2}\left(\frac{\theta}{2}\right)
$$

- If $\tau(\mathbf{x})=c c l w$ and $\tau(\mathbf{y})=c c l w$,

$$
-16 \sin ^{2}\left(\frac{\theta}{2}\right) e^{i \theta}
$$

We now describe what happens when performing an essential move adding the long edge $\left(v_{k}, v_{\ell}\right)$ or $\left(v_{\ell}, v_{k}\right)$. Suppose that the dual edge in $F^{*}$ is oriented as in Case A of Figure 22. We give all details for the first case only, since others are similar.

- If $\tau(\mathbf{x})=c w$ and $\tau(\mathbf{y})=c w$. When the essential move adds the edge $v_{k} v_{\ell}$ in either of the two directions, one of $v_{k}$ or $v_{\ell}$ is a non-root vertex, so that the contribution of $t_{k}$ or $t_{\ell}$ is:

$$
4 i \sin \left(\frac{\theta}{2}\right) e^{-i \frac{\theta}{2}}
$$

By Equation (12), the contribution of the edge $\left(v_{k}, v_{\ell}\right)$ is independent of its orientation and is equal to:

$$
-2 i \sin \theta \text {. }
$$


Suppose that the essential move adds the long edge $\left(v_{k}, v_{\ell}\right)$. Then, by Case $A$ of Figure 22, there are two possible configurations at the triangle $t_{k}$, and the contribution is:

$$
f_{w_{k}} \overline{f_{v_{k}}} K_{w_{k}, v_{k}}+f_{w_{k}} \overline{f_{z_{k}}} K_{w_{k}, z_{k}}=f_{w_{k}} \overline{f_{v_{k}}}-f_{w_{k}} \overline{f_{z_{k}}}=f_{w_{k}} \overline{f_{w_{k}}}=1 .
$$

Suppose that the essential move adds the long edge $\left(v_{\ell}, v_{k}\right)$. Then, using symmetries, we are in Case $B$ of Figure 22. Thus there is one possible configuration at the triangle $t_{\ell}$, and the contribution is:

$$
f_{w_{\ell}} \overline{f_{z_{\ell}}} K_{w_{\ell}, z_{\ell}}=e^{-i \theta} .
$$

As a consequence, the contribution to $\mathcal{W}_{k}$ of the edge added in one of the two possible directions and of the two incidents triangles is

$$
8 \sin \left(\frac{\theta}{2}\right) e^{-i \frac{\theta}{2}} \sin \theta\left(1+e^{-i \theta}\right)=16 \sin \left(\frac{\theta}{2}\right) e^{-i \theta} \sin \theta \cos \left(\frac{\theta}{2}\right) .
$$

- If $\tau(\mathbf{x})=c w$ and $\tau(\mathbf{y})=c c l w$. In a similar way, the contribution to $\mathcal{W}_{k}$ is:

$$
\begin{aligned}
& \left(-4 i \sin \left(\frac{\theta}{2}\right) e^{i \frac{\theta}{2}}\right)(-2 i \sin \theta)(1)+\left(4 i \sin \left(\frac{\theta}{2}\right) e^{-i \frac{\theta}{2}}\right)(-2 i \sin \theta)(-1) \\
& =-16 \sin \left(\frac{\theta}{2}\right) \sin \theta \cos \left(\frac{\theta}{2}\right)
\end{aligned}
$$

- If $\tau(\mathbf{x})=c c l w$ and $\tau(\mathbf{y})=c w$, the contribution to $\mathcal{W}_{k}$ is:

$$
\begin{aligned}
& \left(4 i \sin \left(\frac{\theta}{2}\right) e^{-i \frac{\theta}{2}}\right)(-2 i \sin \theta)\left(-e^{i \theta}\right)+\left(-4 i \sin \left(\frac{\theta}{2}\right) e^{i \frac{\theta}{2}}\right)(-2 i \sin \theta)\left(e^{-i \theta}\right) \\
& =-16 \sin \left(\frac{\theta}{2}\right) \sin \theta \cos \left(\frac{\theta}{2}\right) .
\end{aligned}
$$

- If $\tau(\mathbf{x})=c c l w$ and $\tau(\mathbf{y})=c c l w$, the contribution to $\mathcal{W}_{k}$ is:

$$
\begin{aligned}
& \left(-4 i \sin \left(\frac{\theta}{2}\right) e^{i \frac{\theta}{2}}\right)(-2 i \sin \theta)\left(-e^{i \theta}\right)+\left(-4 i \sin \left(\frac{\theta}{2}\right) e^{i \frac{\theta}{2}}\right)(-2 i \sin \theta)(-1) \\
& =16 \sin \left(\frac{\theta}{2}\right) e^{i \theta} \sin \theta \cos \left(\frac{\theta}{2}\right) .
\end{aligned}
$$

Moreover, by Points 1. -4 . we know that the contribution of all other long edges, inner edges and triangles is the same in $\mathcal{W}_{k}$ and $\mathcal{W}_{k-1}$. Thus, from the above computations we deduce that, independently of the type of the decorations $\mathbf{x}$ and $\mathbf{y}$ :

$$
\begin{aligned}
\frac{\mathcal{W}_{k}}{\mathcal{W}_{k-1}} & =-\frac{16 \sin \left(\frac{\theta}{2}\right) \sin \theta \cos \left(\frac{\theta}{2}\right)}{16 \sin ^{2}\left(\frac{\theta}{2}\right)} \\
& =-\sin \theta \cot \left(\frac{\theta}{2}\right)=-(1+\cos \theta)
\end{aligned}
$$


Let us now introduce the notation $\theta_{i_{j}}$ for the rhombus half-angle of the edge $\mathbf{e}_{i_{j}}$. Then, from Formula (18), we deduce that independently of the type of the vertices:

$$
\mathcal{W}_{k}=\mathcal{W}_{0} \prod_{j=1}^{k}\left(-\left(1+\cos \theta_{i_{j}}\right)\right)
$$

Conclusion. Independently of the type of the vertices, we have:

$$
\begin{aligned}
\sum_{k=0}^{m} \sum_{\left(i_{1}, \cdots, i_{k}\right) \in J_{k}} \mathcal{W}_{k} & =\mathcal{W}_{0} \sum_{k=0}^{m} \sum_{\left(i_{1}, \cdots, i_{k}\right) \in J_{k}} \prod_{j=1}^{k}\left(-\left(1+\cos \theta_{i_{j}}\right)\right) \\
& =\mathcal{W}_{0} \prod_{i=1}^{m}\left[1-\left(1+\cos \theta_{i}\right)\right] \\
& =\mathcal{W}_{0}(-1)^{m} \prod_{i=1}^{m} \cos \theta_{i} \\
& =\mathcal{W}_{0}(-1)^{M-N} \prod_{\mathbf{x y} \in E\left(G_{1}\right) \backslash \mathbf{F}} \cos \theta_{\mathbf{x y}}, \quad(\operatorname{changing} \text { notations }) \\
& =\left[2^{4 M} \prod_{\mathbf{x y} \in E\left(G_{1}\right)} \sin ^{2}\left(\frac{\theta_{\mathbf{x y}}}{2}\right)\right]\left[\prod_{\mathbf{x y} \in \mathbf{F}} \sin \theta_{\mathbf{x y}}\right]\left[\prod_{\mathbf{x y} \in E\left(G_{1}\right) \backslash \mathbf{F}} \cos \theta_{\mathbf{x y}}\right] \\
& =\left[2^{4 M} \prod_{\mathbf{x y} \in E\left(G_{1}\right)} \sin ^{2}\left(\frac{\theta_{\mathbf{x y}}}{2}\right) \cos \theta_{\mathbf{x y}}\right] \prod_{\mathbf{x y} \in \mathbf{F}} \tan \theta_{\mathbf{x y}}
\end{aligned}
$$

Summing over the $2^{N}$ possible types for the vertices of $G_{1}$ yields Theorem 30 .

Corollary 31 ([BdT10b, BdT10a]).

$$
P_{\text {dimer }}(\mathbf{z}, \mathbf{w})=\left(2^{\left|V\left(G_{1}\right)\right|} \prod_{\mathbf{x y} \in E\left(G_{1}\right)}\left[\cot ^{2}\left(\frac{\theta_{\mathbf{x y}}}{2}\right)-1\right]\right) P_{\text {Lap }}(\mathrm{z}, \mathrm{w}) .
$$

Proof. From Theorem 29 and 30, we deduce that:

$$
P_{\text {dimer }}^{0}(\mathrm{z}, \mathrm{w})=\mathbf{C} P_{\text {Lap }}(\mathrm{z}, \mathrm{w}) \text {. }
$$

Moreover, by Equation (5),

$$
P_{\text {dimer }}^{0}(\mathrm{z}, \mathrm{w})=\left(\prod_{x \in V\left(\mathcal{G}_{1}\right)}\left|f_{x}\right|^{2}\right) P_{\text {dimer }}(\mathrm{z}, \mathrm{w}) .
$$

By definition of the vector $f$, for every decoration $\mathbf{x}$ and for every $k \in\left\{1, \cdots, d_{\mathbf{x}}\right\}$ :

$$
\begin{aligned}
\left|f_{w_{k}(\mathbf{x})}\right| & =\left|f_{z_{k}(\mathbf{x})}\right|=1 \\
\left|f_{v_{k}(\mathbf{x})}\right|^{2} & =2(1-\cos \theta)=4 \sin ^{2}\left(\frac{\theta}{2}\right),
\end{aligned}
$$


where $\theta$ is the rhombus half-angle of the long edge incident to $v_{k}(\mathbf{x})$. As a consequence:

$$
\prod_{x \in V\left(\mathcal{G}_{1}\right)}\left|f_{x}\right|^{2}=2^{4 M} \prod_{\mathbf{x y} \in E\left(G_{1}\right)} \sin ^{4}\left(\frac{\theta_{\mathbf{x y}}}{2}\right) .
$$

We conclude:

$$
\begin{aligned}
P_{\text {dimer }}(\mathbf{z}, \mathrm{w}) & =\left(\frac{\mathbf{C}}{\prod_{x \in V\left(\mathcal{G}_{1}\right)}\left|f_{x}\right|^{2}}\right) P_{\text {Lap }}(\mathbf{z}, \mathrm{w}) \\
& =\left(2^{N} \prod_{\mathbf{x y} \in E\left(G_{1}\right)} \frac{\cos \theta_{\mathbf{x y}}}{\sin ^{2}\left(\frac{\theta_{\mathbf{x y}}}{2}\right)}\right) P_{\text {Lap }}(\mathbf{z}, \mathbf{w}) \\
& =\left(2^{N} \prod_{\mathbf{x y} \in E\left(G_{1}\right)}\left[\cot ^{2}\left(\frac{\theta_{\mathbf{x y}}}{2}\right)-1\right]\right) P_{\text {Lap }}(\mathrm{z}, \mathrm{w}) .
\end{aligned}
$$

\section{References}

[Bax86] R. J. Baxter. Free-fermion, checkerboard and $Z$-invariant lattice models in statistical mechanics. Proc. Roy. Soc. London Ser. A, 404(1826):1-33, 1986.

[Bax89] Rodney J. Baxter. Exactly solved models in statistical mechanics. Academic Press Inc. [Harcourt Brace Jovanovich Publishers], London, 1989. Reprint of the 1982 original.

[BdT10a] Cédric Boutillier and Béatrice de Tilière. The critical $Z$-invariant ising model via dimers: Locality property. Communications in Mathematical Physics, pages 1-44, 2010. 10.1007/s00220-010-1151-3.

[BdT10b] Cédric Boutillier and Béatrice de Tilière. The critical $Z$-invariant ising model via dimers: the periodic case. Probability Theory and Related Fields, 147:379-413, 2010. 10.1007/s00440-009-0210-1.

[Fis66] M. E. Fisher. On the dimer solution of planar ising models. Journal of Mathematical Physics, 7:1776-1781, October 1966.

[For93] R. Forman. Determinants of Laplacians on graphs. Topology, 32(1):35-46, 1993.

[Kas61] P. W. Kasteleyn. The statistics of dimers on a lattice : I. the number of dimer arrangements on a quadratic lattice. Physica, 27:1209-1225, December 1961.

[Kas67] P. W. Kasteleyn. Graph theory and crystal physics. In Graph Theory and Theoretical Physics, pages 43-110. Academic Press, London, 1967. 
[Ken02] Richard Kenyon. The laplacian and dirac operators on critical planar graphs. Invent. Math., 150(2):409-439, 2002.

[Mes06] RJ Messikh. The surface tension near criticality of the 2d-Ising model. Arxiv preprint math/0610636, 2006.

[MW73] B. McCoy and F. Wu. The two-dimensional Ising model. Harvard Univ. Press, 1973.

[TF61] HNV Temperley and M.E. Fisher. Dimer problem in statistical mechanics-an exact result. Philosophical Magazine, 6(68):1061-1063, 1961.

[Wil11] David B. Wilson. Xor-ising loops and the gaussian free field. arxiv:1102.3782, 2011. 